\title{
Anomalies of QFTs from M-theory and holography
}

\author{
Ibrahima Bah, ${ }^{a}$ Federico Bonetti, ${ }^{a}$ Ruben Minasian ${ }^{b, c}$ and Emily Nardoni ${ }^{d}$ \\ ${ }^{a}$ Department of Physics and Astronomy, Johns Hopkins University, \\ 3400 North Charles Street, Baltimore, MD 21218, U.S.A. \\ ${ }^{b}$ Institut de Physique Théorique, Université Paris Saclay, CNRS, CEA, \\ F-91191, Gif-sur-Yvette, France \\ ${ }^{c}$ School of Physics, Korea Institute for Advanced Study, \\ Seoul 02455, Korea \\ ${ }^{d}$ Mani L. Bhaumik Institute for Theoretical Physics, Department of Physics and Astronomy, \\ University of California, \\ Los Angeles, CA 90095, U.S.A. \\ E-mail: iboubah@jhu.edu, fbonett3@jhu.edu, ruben.minasian@ipht.fr, \\ enardoni@ucla.edu
}

AbStRaCt: We describe a systematic way of computing the 't Hooft anomalies for continuous symmetries of Quantum Field Theories in even dimensions that can be geometrically engineered from M5-branes. Our approach is based on anomaly inflow, and characterizes the anomaly polynomial of the QFT in terms of the geometric definition of the field theory. In particular, when the QFT admits a holographic dual, the topological data of the solution is sufficient to compute the anomalies of the dual field theory, including finite terms in $N$. We study several classes of examples in four and six dimensions, with or without known M5-brane probe configurations.

Keywords: Anomalies in Field and String Theories, M-Theory

ArXIV EPRINT: 1910.04166 


\section{Contents}

1 Introduction 1

2 General aspects of anomaly inflow in M-theory 3

2.1 Anomaly inflow for wrapped M5-branes 3

2.2 Applications to holography 5

3 Construction of $\boldsymbol{E}_{4} \quad 6$

3.1 Parametrization of $E_{4} \quad 7$

$\begin{array}{ll}3.2 \text { Deformations of } E_{4} & 10\end{array}$

4 Examples in six dimensions $\quad 11$

4.1 A stack of $N$ M5-branes 12

4.2 M5-branes probing an ADE singularity 13

5 Examples in four dimensions $\quad 16$

$\begin{array}{lll}5.1 & S^{4} \text { fibrations over a smooth Riemann surface } & 16\end{array}$

$\begin{array}{ll}\text { 5.1.1 Topology and isometries of } M_{6} & 16\end{array}$

$\begin{array}{ll}\text { 5.1.2 Aside on terminology: twisting vs gauging } & 18\end{array}$

$\begin{array}{ll}5.1 .3 \text { Construction of } E_{4} & 18\end{array}$

$\begin{array}{ll}\text { 5.1.4 Computation of } X_{8} & 20\end{array}$

5.1.5 Inflow anomaly polynomial 21

$5.2 S^{2}$ fibrations over a product of Riemann surfaces 22

$\begin{array}{llr}6 & \text { Discussion } & 27\end{array}$

$\begin{array}{ll}\text { A Some useful identities } & 29\end{array}$

$\begin{array}{lll}\text { A.1 Identities for gauging of isometries } & 29\end{array}$

A.2 Identities for $\mathrm{SO}(5)$ isometry of $S^{4} \quad 30$

B The $G$-equivariant cohomology class defined by $E_{4} \quad 31$

B.1 Relation between $V_{4}^{\mathrm{eq}}, \omega_{\alpha}^{\mathrm{eq}}$ and $G$-equivariant polyforms 31

B.2 Deformations of $E_{4}$ and $G$-equivariant cohomology 32

C (In)dependence of $I_{d+2}^{\text {inflow }}$ on $\gamma_{4}$

$\begin{array}{lll}\text { C.1 The case } d=6 & 35\end{array}$

$\begin{array}{lll}\text { C.2 } & \text { The case } d=4 & 36\end{array}$

$\begin{array}{lll}\text { C.3 The case } d=2 & 38\end{array}$

$\begin{array}{ll}\text { D Details on branes at an orbifold singularity } & 39\end{array}$

E Details on the BBBW setup $\quad 42$ 
$\begin{array}{lll}\text { F Details on the GMSW setup } & 46\end{array}$

F.1 Brief review of the solutions 46

F.2 Details on the construction of $E_{4}$ for GMSW 48

\section{Introduction}

Geometric engineering is a powerful way to study Quantum Field Theories (QFTs) and their various dynamics. Many interesting QFTs can be explored by studying the low energy limit of branes in string theory backgrounds. Some of the most interesting and yet mysterious QFTs have such a definition: for instance, the $A_{N-1} \mathcal{N}=(2,0)$ superconformal field theories in six dimensions have a description as the worldvolume theories on a stack of $N$ flat M5-branes [1, 2]; and a class of $6 \mathrm{~d}$ theories with reduced $(1,0)$ supersymmetry are obtained from M5-branes probing an orbifold singularity [3]. Further wrapping the branes on a compact manifold yields a large class of lower dimensional QFTs. For example, by reducing the $(2,0)$ theories on a Riemann surface we obtain a large class of generically strongly coupled 4d QFTs of varying amounts of supersymmetry known as Class $\mathcal{S}$, first analyzed for $\mathcal{N}=2$ theories in $[4,5]$, and studied for $\mathcal{N}=1$ cases in [6-10]. Embedding these systems in string theory provides an organizing principle and geometric toolset for exploring their properties, especially in their strong coupling regimes.

An important problem in these constructions is to compute the anomalies of the field theories. ${ }^{1}$ 't Hooft anomalies provide a robust set of observables that are useful for probing the dynamics of QFTs. They are preserved under renormalization group flow, and then can be used to constrain the IR phases of quantum systems via anomaly matching. For a superconformal field theory, the 't Hooft anomalies involving R-symmetry are related to central charges by the superconformal algebra [11, 12]. Anomalies are also naturally geometric quantities. For the case of continuous symmetries in even $d$-dimensional QFTs, they are encoded in the anomaly polynomial, a $(d+2)$-form polynomial in curvature forms associated to gauge and gravity fields [13-15]. For QFTs obtained by dimensional reduction of a higher dimensional field theory, anomaly matching is implemented by integrating the upstairs anomaly polynomial over the compact manifold in the reduction. However, this prescription only gives the contribution to the lower dimensional anomaly polynomial that derives from symmetries manifest in the higher dimensional theory — it is not sensitive to decoupled sectors, accidental symmetries, and other subtleties.

The primary objective of this work is to provide a systematic way of computing the anomalies of geometrically engineered QFTs in $d$ dimensions from M5-branes. Our main tool is anomaly inflow in the M-theory background, first studied for M5-branes in [16-19]. The M5-branes act as a singular magnetic source for the M-theory 4-form flux $G_{4}$. In the supergravity description we excise a small neighborhood of the stack, thus inducing

\footnotetext{
${ }^{1}$ Throughout we restrict to the case of anomalies in background rather than dynamical gauge symmetries ('t Hooft anomalies), so that their existence does not render the theory inconsistent.
} 
a boundary for 11d spacetime. For the geometries under consideration, this boundary is a fibration of an internal space $M_{10-d}$ over the low energy QFT worldvolume $W_{d}$. The global symmetries of the QFT are fixed by isometries of the internal space, as well as gauge symmetries of the three-form potential. In inflow, the anomalies for degrees of freedom on the branes must cancel the classical anomalous variation of the effective 11d supergravity action localized on the branes. The anomalous variation of the action is related by descent relations to a 12 -form characteristic class $\mathcal{I}_{12}$. Reducing $\mathcal{I}_{12}$ along the transverse directions to the QFT worldvolume yields the inflow anomaly polynomial $I_{d+2}^{\text {inflow }}$ associated to the QFT. Then, the anomalies of the QFT are equal to those computed via inflow up to decoupling modes, such that $I_{d+2}^{\text {inflow }}+I_{d+2}^{\text {QFT }}+I_{d+2}^{\text {decoupl }}=0$.

We provide a general prescription for computing $\mathcal{I}_{12}$, and describe its properties and uniqueness. The essential point is that $\mathcal{I}_{12}$ is determined entirely by topological properties of the internal space $M_{10-d}$, and equivariant classes constructed from the boundary data of the 4-form flux. We see in examples of dimensionally reduced theories that the procedure of directly integrating $\mathcal{I}_{12}$ to compute $I_{d+2}^{\text {inflow }}$ can contain more information than the reduction of the anomaly polynomial of the parent theory. Further, the embedding in M-theory may allow for a geometric interpretation of the decoupling modes.

We will then examine the implications of this machinery for holography, i.e. when the $d$-dimensional QFT is a conformal field theory with a large- $N A d S_{d+1}$ gravity dual $(N$ being the number of M5-branes). The dual geometry in M-theory consists of a warped product of $A d S_{d+1}$ times $M_{10-d}^{\text {hol }}$, supported by a $G_{4}^{\text {hol }}$ flux configuration on the internal space. The transverse directions $M_{10-d}$ to the QFT worldvolume are identified with the internal space $M_{10-d}^{\text {hol }}$. The main observation is that we can use a known solution of $11 \mathrm{~d}$ supergravity to infer $\mathcal{I}_{12}$. This is because (1) the topology of the internal space $M_{10-d}^{\text {hol }}$ is the same as that of $M_{10-d}$, and (2) the holographic $G_{4}^{\text {hol }}$ flux configuration can be identified with our seed boundary $G_{4}$-flux utilized in the inflow machinery.

The power of our method in the context of holography is twofold. First, since the seed topological data for inflow can be read off of a known supergravity solution, we can obtain the CFT anomalies even if we don't know the probe M5-brane configuration. We demonstrate this below by applying our method to the $A d S_{5} \times M_{6}$ Gauntlett-MartelliSparks-Waldram (GMSW) solutions [20]. Second, this prescription provides a path from the classical solution of 2-derivative supergravity which is valid at large- $N$, to the exact anomaly polynomial via inflow. Since our $\mathcal{I}_{12}$ involves higher derivative terms inherited from the M-theory action, our prescription captures contributions to the anomalies at finite $N$. In examples, we correctly produce all $N$-dependent anomaly coefficients excluding $\mathcal{O}\left(N^{0}\right)$ terms which we can identify with decoupling modes.

We demonstrate our method in several examples, some of which have an explicit brane construction, and some cases in which only the holographic solution is known. In section 4, we focus on QFTs in six dimensions. First we exemplify our method in the case of flat M5-branes. Of course, anomaly inflow for this setup is well-known $[18,19],{ }^{2}$ and we use this

\footnotetext{
${ }^{2}$ The anomaly polynomials for general $6 \mathrm{~d}(2,0)$ ADE theories can be computed using anomaly matching on the tensor branch [21], while inflow for the $D_{N}$ series is studied in [22]. Also note that the holographic computation of the $c$ central charge is given in [23].
} 
analysis mainly to set notation for subsequent examples. We then apply our method to the case of M5-branes probing a $\mathbb{C}^{2} / \Gamma$ singularity, for $\Gamma$ an ADE subgroup of $\mathrm{SU}(2)$. We match the anomaly polynomial for these $\mathcal{N}=(1,0)$ theories as given in [24], where an analysis via anomaly matching on the tensor branch as well as an inflow analysis appears. One comment is that our analysis produces an additional term relative to the inflow results from [24] that corresponds precisely to the contribution of the Green-Schwarz term associated to the center of mass mode for the branes. For the case of $\Gamma=\mathbb{Z}_{k}$, we reproduce the full result of [25].

In section 5, we consider two classes of four-dimensional $\mathcal{N}=1$ QFTs. First, we reproduce the anomalies of $4 \mathrm{~d} \mathcal{N}=1$ SCFTs for which the internal space $M_{6}$ is an $S^{4}$ fibration over a smooth Riemann surface, analyzed by Bah-Beem-Bobev-Wecht (BBBW) [9, 10]. We then apply our method to a class of $4 \mathrm{~d}$ SCFTs where $M_{6}$ is an $S^{2}$ fibration over a product of smooth Riemann surfaces, corresponding to the GMSW supergravity solutions [20]. The M5-brane probe description is generally not known for these solutions, but we are nonetheless able to compute the inflow anomaly polynomial, and show that we match the holographic computation of the central charge [26]. Our results are the first computation of the subleading-in- $N$ corrections to the central charges of this class of QFTs.

We conclude with a discussion of our results, as well as several appendices that contain the details of computations that appear in the main text.

\section{General aspects of anomaly inflow in M-theory}

In this section we discuss general aspects of anomaly inflow for M-theory setups with wrapped M5-branes. We establish a connection to holography and outline a general recipe for obtaining the inflow anomaly polynomial. The latter is governed by a 4 -form $E_{4}$ that encodes topological information about the $G_{4}$-flux configuration. In this section we discuss general properties of $E_{4}$ and all the necessary ingredients for its construction.

\subsection{Anomaly inflow for wrapped M5-branes}

Let us consider a stack of $N$ M5-branes with worldvolume $W_{6}$. The tangent bundle of the 11d ambient space $M_{11}$, restricted to the worldvolume $W_{6}$, decomposes according to

$$
\left.T M_{11}\right|_{W_{6}}=T W_{6} \oplus N W_{6},
$$

where $T W_{6}$ is the tangent bundle to the stack and $N W_{6}$ is the normal bundle to the stack. The latter has structure group $\mathrm{SO}(5)$ and encodes the five transverse directions of the M5-branes.

We are interested in setups in which

$$
W_{6}=W_{d} \times \mathcal{S}_{6-d},
$$

where $W_{d}$ is external $d$-dimensional spacetime and $\mathcal{S}_{6-d}$ is a smooth compact evendimensional internal space. At low energies the system is described by a QFT living on $W_{d}$. For $d=6$, the internal space is understood to be absent. For $d<6$, in order to specify the setup we have to describe the topology of the normal bundle $N W_{6}$ over the internal space 
$\mathcal{S}_{6-d}$. This amounts to specifying a partial topological twist of the worldvolume theory living on the M5-branes upon compactification on $\mathcal{S}_{6-d}$. The topological twist is essential in preserving some supersymmetry in the $d$ non-compact directions.

Given the internal space $\mathcal{S}_{6-d}$ wrapped by the M5-branes and the $S^{4}$ that surrounds the stack in its five transverse directions, there is a compact $(10-d)$-dimensional space $M_{10-d}$ that encodes the topological twist and governs the anomalies of the QFT on $W_{d}$. The space $M_{10-d}$ is an $S^{4}$ fibration over $\mathcal{S}_{6-d}$,

$$
S^{4} \hookrightarrow M_{10-d} \rightarrow \mathcal{S}_{6-d}
$$

The structure group of the fibration (2.3) is a subgroup of the $\mathrm{SO}(5)$ structure group of the normal bundle $N W_{6}$.

The QFT living on $W_{d}$ at low energies can admit 't Hooft anomalies for the global symmetries of the theory. In this work we focus on continuous symmetries. Their anomalies are diagnosed by coupling the QFT on $W_{d}$ to background gauge connections and metric.

Since the full M-theory setup is anomaly-free, the 't Hooft anomalies in $d$ dimensions must be counterbalanced by anomaly inflow from the M-theory bulk, which is analyzed using the methods of $[18,19]$. The M5-brane stack acts as a singular magnetic source for the M-theory $G_{4}$ flux. To describe the setup in supergravity, the singularity is removed by excising a small tubular neighborhood of the M5-brane stack. As a result, the 11d spacetime $M_{11}$ acquires a boundary $\partial M_{11}=M_{10}$. If $r$ denotes the radial coordinate away from the M5-brane stack, $M_{10}$ is located at $r=\epsilon$, where $\epsilon$ is a small positive constant. The space $M_{10}$ is a fibration of $M_{10-d}$ over $W_{d}$,

$$
M_{10-d} \hookrightarrow M_{10} \rightarrow W_{d} .
$$

The fibration (2.4) is specified by the background gauge connections for the symmetries of the QFT that originate from continuous isometries of $M_{10-d}$. Let us stress that the fibration (2.4) encodes the gauging of the $d$-dimensional theory with background gauge fields on $W_{d}$, while the fibration (2.3) describes the topological twist that defines the theory on $W_{d}$.

The magnetic source for $G_{4}$ is modeled by imposing suitable boundary conditions at $r=\epsilon$. More precisely, we have

$$
\left.\frac{G_{4}}{2 \pi}\right|_{r=\epsilon}=E_{4}
$$

where $E_{4}$ is a closed 4 -form on the space $M_{10}$. The relation (2.5) is written in conventions in which $G_{4}$-flux quantization reads

$$
\int_{\mathcal{C}_{4}}\left(\frac{G_{4}}{2 \pi}-\frac{\lambda}{2}\right) \in \mathbb{Z}
$$

where $\mathcal{C}_{4}$ is a 4 -cycle and $\lambda=p_{1}\left(T M_{11}\right) / 2[27]$. (In all setups we consider $\lambda / 2$ is integral.) The 4-form $E_{4}$ has to be globally defined and invariant under the structure group of the fibration (2.4), i.e. invariant under all symmetries of the $d$-dimensional theory. Moreover, we have

$$
\int_{S^{4}} E_{4}=N
$$


where $S^{4}$ is the 4 -sphere surrounding the stack. Depending on the choice of $\mathcal{S}_{6-d}$ and the topology of the fibration (2.3), the 4 -form $E_{4}$ might have additional non-trivial fluxes through 4-cycles in $M_{10}$, besides (2.7).

As explained in $[18,19]$, in the presence of the boundary $M_{10}$ the topological terms in the low-energy effective action of M-theory $S_{11 d}$ are no longer invariant under gauge transformations in the background connections on $W_{d}$. In fact, we have

$$
\frac{\delta S_{11 d}}{2 \pi}=\int_{M_{10}} \mathcal{I}_{10}^{(1)}
$$

where $\mathcal{I}_{10}^{(1)}$ is a 10 -form on $M_{10}$, linear in the gauge parameters. In accordance with the Wess-Zumino consistency conditions, $\mathcal{I}_{10}^{(1)}$ is related by descent to a 12 -form characteristic class $\mathcal{I}_{12}$,

$$
d \mathcal{I}_{10}^{(1)}=\delta \mathcal{I}_{11}^{(0)}, \quad d \mathcal{I}_{11}^{(0)}=\mathcal{I}_{12}
$$

The class $\mathcal{I}_{12}$ is given by

$$
\mathcal{I}_{12}=-\frac{1}{6} E_{4}^{3}-E_{4} X_{8}
$$

where we have suppressed wedge products. The 8 -form $X_{8}$ is given by

$$
X_{8}=\frac{1}{192}\left[p_{1}\left(T M_{11}\right)^{2}-4 p_{2}\left(T M_{11}\right)\right],
$$

where the quantities $p_{1,2}\left(T M_{11}\right)$ are the first and second Pontryagin classes of the $11 \mathrm{~d}$ tangent bundle, implicitly pulled back to the boundary at $r=\epsilon$.

If we integrate the class $\mathcal{I}_{12}$ on the $M_{10-d}$ fibers of (2.4), we obtain the $(d+2)$-form inflow anomaly polynomial of the $d$-dimensional theory on $W_{d}$,

$$
I_{d+2}^{\text {inflow }}=\int_{M_{10-d}} \mathcal{I}_{12}
$$

The inflow anomaly polynomial (2.12) cancels against the 't Hooft anomalies of the interacting QFT living on $W_{d}$ at low energies, and of the decoupling modes related to the center-of-mass of the M5-brane stack. We thus write

$$
I_{d+2}^{\text {inflow }}+I_{d+2}^{\mathrm{QFT}}+I_{d+2}^{\text {decoupl }}=0 .
$$

\subsection{Applications to holography}

One of the main interests of this work is the case in which the interacting QFT on $W_{d}$ is a CFT with a gravity dual. The dual geometry in M-theory is a warped product of $A d S_{d+1}$ with an internal $(10-d)$-dimensional space,

$$
M_{11}=A d S_{d+1} \times_{w} M_{10-d}^{\text {hol }} .
$$

This $A d S_{d+1}$ solution is supported by a non-trivial $G_{4}$-flux configuration $G_{4}^{\text {hol }}$ on the internal space $M_{10-d}^{\text {hol }}{ }^{3}$

\footnotetext{
${ }^{3}$ For $d=2, G_{4}^{\text {hol }}$ can have additional terms with three external legs, proportional to the volume form on $A d S_{3}$. Such terms in $G_{4}^{\text {hol }}$ do not play a role in the following discussion.
} 
The main observation is that, for $A d S_{d+1}$ solutions that correspond to wrapped M5branes, the topology of the internal space $M_{10-d}^{\text {hol }}$ is the same as the topology of the space $M_{10-d}$ defined in (2.3) [28]. By a similar token, the holographic $G_{4}$-flux configuration $G_{4}^{\text {hol }} /(2 \pi)$ lies in the same cohomology class as $E_{4}$ after all external connections are turned off,

$$
\frac{G_{4}^{\mathrm{hol}}}{2 \pi}=\left.E_{4}\right|_{\text {external connections }=0} \quad \text { in cohomology of } M_{10-d} .
$$

Let us emphasize that the topological properties of $M_{10-d}$ and $E_{4}$ are the main ingredients in the implementation of anomaly inflow for wrapped M5-branes. The discussion above indicates that these topological features can be equivalently extracted from the probe setup or from the holographic solution.

There exists a larger set of $A d S_{d+1}$ solutions in M-theory for which the probe M5-brane configuration is not known. In these solutions the internal space $M_{10-d}^{\text {hol }}$ is not necessarily an $S^{4}$ fibration over some $(6-d)$-dimensional space, as in (2.3). We expect that our method for the computation of $I_{d+2}^{\text {inflow }}$ applies to such setups. The anomaly is governed by the topological properties of $M_{10-d}^{\text {hol }}$ and $G_{4}^{\text {hol }}$, which determine $E_{4}$.

The general task at hand is the construction of $E_{4}$ given the topology of the space $M_{10-d}$. Recall that $E_{4}$ is a 4 -form on the total space $M_{10}$ of the fibration (2.4). Crucially, we do not assume that $M_{10-d}$ is an $S^{4}$ fibration over a $(6-d)$-dimensional space. As a result, the following considerations apply beyond setups that are realized by wrapping M5-branes on a smooth compact internal space.

A local representative for the class $E_{4}$ is constrained by the following properties:

- $E_{4}$ is globally defined,

- $E_{4}$ is closed,

- $E_{4}$ is invariant under all symmetries of $M_{10-d}$.

The 4 -form $E_{4}$ is constructed by combining the curvatures of the background connections on $W_{d}$ with $p$-forms in the internal space $M_{10-d}$. Crucially, since $M_{10-d}$ is fibered over $W_{d}$, see (2.4), the internal $p$-forms on $M_{10-d}$ must be appropriately "gauged", i.e. coupled to the background connections on $W_{d}$.

The constraints listed above may not completely fix the expression of $E_{4}$. In section 3 we present a general recipe for the construction of $E_{4}$, we characterize its ambiguities, and we argue that they do not affect the inflow anomaly polynomial.

\section{Construction of $\boldsymbol{E}_{4}$}

In this section we introduce a convenient formalism for the parametrization of the 4 -form $E_{4}$. We show how to construct a good representative for $E_{4}$ in terms on $p$-forms in $M_{10-d}$. The natural language to describe this construction is that of $G$-equivariant cohomology. While $E_{4}$ is generically non-unique, we argue that the inflow anomaly polynomial can be extracted unambiguously. 


\subsection{Parametrization of $E_{4}$}

A local representative for the class $E_{4}$ is a closed, gauge-invariant, globally-defined 4-form on the total space $M_{10}$ of the fibration of $M_{10-d}$ over external spacetime $W_{d}$, see (2.4). The 4-form $E_{4}$ is constructed using internal $p$-forms on the $M_{10-d}$ fibers, together with external curvatures with legs on $W_{d}$.

We suppose that $M_{10-d}$ admits a collection of Killing vectors $k_{I}^{m}$, with $m$ a curved index on $M_{10-d}$, and $I$ labeling a basis of Killing vectors. The latter obey the Lie algebra

$$
£_{I} k_{J} \equiv £_{k_{I}} k_{J}=\left[k_{I}, k_{J}\right]=f_{I J}{ }^{K} k_{K},
$$

where $£$ denotes Lie derivative. The non-trivial fibration of $M_{10-d}$ over $W_{d}$ is encoded by the gauging of the isometries of $M_{10-d}$. In what follows, we adopt a notation similar to the one of [29]. The gauging is conveniently described locally by the replacement

$$
d \xi^{m} \rightarrow D \xi^{m}=d \xi^{m}+k_{I}^{m} A^{I}
$$

where $A^{I}$ is the external connection associated to the Killing vector $k_{I}^{m}$. In our conventions, the field strength $F^{I}$ of the connection $A^{I}$ reads

$$
F^{I}=d A^{I}-\frac{1}{2} f_{J K}^{I} A^{J} A^{K} .
$$

Let $\omega$ be a $p$-form on $M_{10-d}$,

$$
\omega=\frac{1}{p !} \omega_{m_{1} \ldots m_{p}} d \xi^{m_{1}} \ldots d \xi^{m_{p}}
$$

where the components $\omega_{m_{1} \ldots m_{p}}$ depend only on the coordinates $\xi$ on $M_{10-d}$. We use the symbol $\omega^{\mathrm{g}}$ for the gauged version of $\omega$, obtained by means of the replacement (3.2),

$$
\omega^{\mathrm{g}}=\frac{1}{p !} \omega_{m_{1} \ldots m_{p}} D \xi^{m_{1}} \ldots D \xi^{m_{p}} .
$$

Further details about this gauging procedure are collected in appendix A.

If we turn off all external connections, $E_{4}$ reduces to a 4 -form denoted $V_{4}$. The latter must be closed and invariant under all the isometries of $M_{10-d}$. Furthermore, $G_{4}$-flux quantization requires the class of $V_{4}$ to be integral. Let $\mathcal{V}^{\Lambda}, \Lambda=1, \ldots, b^{4}\left(M_{10-d}\right)$ be a basis of the integral cohomology group $H^{4}\left(M_{10-d}, \mathbb{Z}\right) .{ }^{4}$ We can expand the cohomology class of $V_{4}$ as

$$
V_{4}=N_{\Lambda} \mathcal{V}^{\Lambda}
$$

with the integers $N_{\Lambda}$ specifying the topology of the $G_{4}$-flux configuration, which is part of the input data that defines the setup and is held fixed throughout the construction of $E_{4}$.

The first step in the parametrization of $E_{4}$ is promoting $V_{4}$ to a closed and gaugeinvariant object. The completion of $V_{4}$ is denoted $V_{4}^{\text {eq }}$ and is given by

$$
V_{4}^{\mathrm{eq}}=V_{4}^{\mathrm{g}}+F^{I} \omega_{I}^{\mathrm{g}}+F^{I} F^{J} \sigma_{I J} .
$$

\footnotetext{
${ }^{4}$ For $d=4$, the internal space is six dimensional and 4-cycles are dual to 2-cycles and harmonic 2-forms; the label $\Lambda$ coincides with $\alpha$ in this case.
} 
In the previous expression, $\omega_{I}$ are 2 -forms on $M_{10-d}$, while $\sigma_{I J}$ are 0 -forms. The superscript 'g' refers to the gauging prescription defined in (3.5), while 'eq' stands for equivariant, for reasons outlined below. The 4 -form $V_{4}^{\text {eq }}$ must be invariant under the gauge transformations associated to the isometries of $M_{10-d}$. Let $\delta_{\lambda} V_{4}^{\text {eq }}$ denote the gauge variation of $V_{4}^{\text {eq }}$, with $\lambda^{I}$ denoting the gauge parameters. In computing $\delta_{\lambda} V_{4}^{\text {eq }}$, we encounter variations of external curvatures, and variations of gauged internal forms. The former are given by the usual expression,

$$
\delta_{\lambda} F^{I}=-f_{J K}{ }^{I} \lambda^{J} F^{K} .
$$

The gauge variations of gauged internal forms are given in terms of the Lie derivative with respect to the Killing vector fields. For example,

$$
\delta_{\lambda}\left(\omega_{I}^{\mathrm{g}}\right)=\lambda^{J}\left(£_{J} \omega_{I}\right)^{\mathrm{g}} .
$$

We refer the reader to appendix A for a more detailed explanation of this point. The requirement $\delta_{\lambda} V_{4}^{\text {eq }}=0$ translates into the conditions

$$
£_{I} V_{4}=0, \quad £_{I} \omega_{J}=f_{I J}{ }^{K} \omega_{K}, \quad £_{I} \sigma_{J_{1} J_{2}}=f_{I J_{1}}{ }^{K} \sigma_{K J_{2}}+f_{I J_{2}}{ }^{K} \sigma_{J_{1} K} .
$$

We also have to demand $d\left(V_{4}^{\mathrm{eq}}\right)=0$. In computing the external derivative of $V_{4}^{\text {eq }}$, the following identity is useful,

$$
d\left(\omega_{I}^{\mathrm{g}}\right)+A^{J}\left(£_{J} \omega_{I}\right)^{\mathrm{g}}=\left(d \omega_{I}\right)^{\mathrm{g}}+F^{J}\left(\iota_{J} \omega_{I}\right)^{\mathrm{g}} .
$$

The quantity on the 1.h.s. is the natural covariant derivative acting on a gauged internal form, since the gauge algebra acts via Lie derivative along Killing vector fields. Accordingly, the r.h.s. is a manifestly gauge invariant quantity. Even though we have written (3.11) for $\omega_{I}$, a similar identity holds for any internal gauged form. Further details on this point can be found in appendix A. Making use of (3.11) and similar identities, together with the Bianchi identity for $F^{I}$, we verify that requirement $d\left(V_{4}^{\text {eq }}\right)=0$ implies the following conditions,

$$
d V_{4}=0, \quad \iota_{I} V_{4}+d \omega_{I}=0, \quad \iota_{(I} \omega_{J)}+d \sigma_{I J}=0 .
$$

The symbol $\iota_{I}$ is a shorthand notation for the interior product $\iota_{k_{I}}$ of a $p$-form with the Killing vector $k_{I}^{m}$.

So far, we have only discussed the external connections associated to isometries of $M_{10-d}$. Another class of external connections is related to harmonic 2-forms on $M_{10-d}$. If $\omega_{\alpha}, \alpha=1, \ldots, b^{2}\left(M_{10-d}\right)$, is a basis of harmonic 2-forms, expansion of the M-theory 3 -form potential onto $\omega_{\alpha}$ yields external vectors $A^{\alpha}$, according to the schematic relation $C_{3}=A^{\alpha} \omega_{\alpha}$. The connections $A^{\alpha}$ are Abelian and have field strength $F^{\alpha}=d A^{\alpha}$. Notice that, for $d=4$, one linear combination of the vectors $A^{\alpha}$ is massive and does not correspond to a symmetry of the system. This point is addressed in greater detail in appendix C.2.

Each harmonic 2-form $\omega_{\alpha}$ is closed and invariant under the action of all isometries of $M_{10-d} \cdot{ }^{5}$ As a result, we can complete $\omega_{\alpha}$ to a closed and gauge-invariant object, denoted

\footnotetext{
${ }^{5}$ The fact that $£_{I} \omega_{\alpha}=0$ can be seen as follows. From $d \omega_{\alpha}=0$ we derive $£_{I} \omega_{\alpha}=d\left(\iota_{I} \omega_{\alpha}\right)$. Making use of $\nabla_{(m} k_{I \mid n)}=0$ and $\nabla^{m} \omega_{\alpha m n}=0$, we verify $\left(£_{I} \omega_{\alpha}\right)_{m n}=\nabla^{p}\left(k_{I} \wedge \omega_{\alpha}\right)_{p m n}$. We have thus established that the 2 -form $£_{I} \omega_{\alpha}$ is both exact and co-exact. It follows that $\int_{M_{10-d}}\left(£_{I} \omega_{\alpha}\right) *\left(£_{I} \omega_{\alpha}\right)=0$ (no sum over $\alpha$, $I$ ), which in turn guarantees $£_{I} \omega_{\alpha}=0$.
} 
$\omega_{\alpha}^{\mathrm{eq}}$. It is given by

$$
\omega_{\alpha}^{\mathrm{eq}}=\omega_{\alpha}^{\mathrm{g}}+2 F^{I} \sigma_{I \alpha},
$$

where $\sigma_{I \alpha}$ are suitable 0 -forms on $M_{10-d}$, and the factor of 2 is inserted for later convenience. We demand $\delta_{\lambda} \omega_{\alpha}^{\mathrm{eq}}=0$, such that

$$
£_{I} \omega_{\alpha}=0, \quad £_{I} \sigma_{J \alpha}=f_{I J}{ }^{K} \sigma_{K \alpha} .
$$

Moreover, we need $d\left(\omega_{\alpha}^{\mathrm{eq}}\right)=0$, which is equivalent to

$$
d \omega_{\alpha}=0, \quad \frac{1}{2} \iota_{I} \omega_{\alpha}+d \sigma_{I \alpha}=0 .
$$

We are now in a position to write down the most general parametrization of $E_{4}$. It reads

$$
E_{4}=V_{4}^{\mathrm{eq}}+\frac{F^{\alpha}}{2 \pi} \omega_{\alpha}^{\mathrm{eq}}+\gamma_{4}
$$

where $V_{4}^{\text {eq }}$ is as in (3.7), $\omega_{\alpha}^{\text {eq }}$ is as in (3.13), while $\gamma_{4}$ denotes an arbitrary closed and gaugeinvariant 4 -form with purely external legs. In appendix $\mathrm{C}$ we show that for $d=2$ and $d=4$ the inflow anomaly polynomial is insensitive to the 4 -form $\gamma_{4}$, which may then be set to zero. For $d=6$, the inflow anomaly polynomial does depend on $\gamma_{4}$. In appendix $\mathrm{C}$ we argue that the correct value of $\gamma_{4}$ is obtained by extremizing the inflow anomaly polynomial. This prescription is equivalent to demanding that the 8 -form $E_{4}^{2}+2 X_{8}$ be trivial in the cohomology of $M_{4}$. We interpret this requirement on $E_{4}^{2}+2 X_{8}$ as a tadpole cancellation condition in M-theory, which must be satisfied in order to have a consistent setup. In section 4 we verify that our treatment of $\gamma_{4}$ gives the correct inflow anomaly in two examples, a flat stack of M5-branes, and M5-branes probing a $\mathbb{C}^{2} / \Gamma$ singularity.

The quantities $V_{4}^{\mathrm{eq}}$ and $\omega_{\alpha}^{\mathrm{eq}}$ introduced above admit a natural interpretation in terms of $G$-equivariant cohomology of $M_{10-d}$, where $G$ is the isometry group of $M_{10-d}$. This justifies the label 'eq'. In appendix B we show how the objects $V_{4}^{\text {eq }}, \omega_{\alpha}^{\text {eq }}$ can be identified with $G$-equivariantly closed (poly)forms on $M_{10-d}$, which specify non-trivial $G$-equivariant cohomology classes.

Let us close this section by introducing a more compact notation, which is sometimes convenient in what follows. Let $X=(I, \alpha)$ be a collective index that unifies external connections originating from isometries of $M_{10-d}$ and external connections associated to harmonic 2 -forms on $M_{10-d}$. We can cast $E_{4}$ in (3.16) in the form

$$
E_{4}=V_{4}^{\mathrm{g}}+F^{X} \omega_{X}^{\mathrm{g}}+F^{X} F^{Y} \sigma_{X Y}+\gamma_{4},
$$

with the identifications

$$
F^{X}=\left(F^{I}, \frac{1}{2 \pi} F^{\alpha}\right), \quad \omega_{X}=\left(\omega_{I}, \omega_{\alpha}\right), \quad \sigma_{X Y}=\left(\begin{array}{cc}
\sigma_{I J} & \sigma_{I \beta} \\
\sigma_{J \alpha} & 0
\end{array}\right) .
$$

By a similar token, we can summarize (3.10) and (3.14) by writing

$$
£_{X} V_{4}=0, \quad £_{X} \omega_{Y}=f_{X Y}{ }^{Z} \omega_{Z}, \quad £_{X} \sigma_{Y_{1} Y_{2}}=f_{X Y_{1}}{ }^{Z} \sigma_{Z Y_{2}}+f_{X Y_{2}}{ }^{Z} \sigma_{Y_{1} Z},
$$


with the understanding that $£_{\alpha} \equiv 0$, and that the only non-zero entry of $f_{X Y}{ }^{Z}$ are $f_{I J}{ }^{K}$. In a similar fashion, we summarize (3.12), (3.15) as

$$
d V_{4}=0, \quad \iota_{X} V_{4}+d \omega_{X}=0, \quad \iota_{(X} \omega_{Y)}+d \sigma_{X Y}=0,
$$

with the convention $\iota_{\alpha} \equiv 0$.

It is interesting to note that the compact expression (3.17) is suggestive of a possible extension of the $G$-equivariant cohomology interpretation of appendix B. Indeed, we can formally augment the isometry group $G$ to a larger group $\widehat{G}$, by adding an extra $\mathrm{U}(1)$ factor for each curvature $F^{\alpha}$. The extra U(1) generators act trivially on $M_{6}$, because of $\iota_{\alpha} \equiv 0$. We can then interpret the quantity $V_{4}^{\mathrm{g}}+F^{X} \omega_{X}^{\mathrm{g}}+F^{X} F^{Y} \sigma_{X Y}$ as a $\widehat{G}$-equivariant (as opposed to $G$-equivariant) completion of $V_{4}$.

\subsection{Deformations of $E_{4}$}

An important question concerning the construction of $E_{4}$ is to determine how uniquely this object is fixed by the conditions (3.10), (3.12), (3.14), (3.15). We refer the reader to appendix B.2 for a detailed analysis of this problem, and here we discuss only some salient aspects.

A class of deformations of $E_{4}$ is of the form

$$
\begin{aligned}
V_{4} \rightarrow V_{4}+d W_{3}, & \omega_{I} \rightarrow \omega_{I}+\iota_{I} W_{3}+d \lambda_{I}, & \sigma_{I J} & \rightarrow \sigma_{I J}+\iota_{(I} \lambda_{J}, \\
\omega_{\alpha} & \rightarrow \omega_{\alpha}+d \lambda_{\alpha}, & \sigma_{I \alpha} & \rightarrow \sigma_{I \alpha}+\frac{1}{2} \iota_{I} \lambda_{\alpha} .
\end{aligned}
$$

where $W_{3}$ is a globally-defined 3-form on $M_{10-d}$, and $\lambda_{I}, \lambda_{\alpha}$ are globally-defined 1-forms on $M_{10-d}$. Gauge-invariance requires

$$
£_{I} W_{3}=0, \quad £_{I} \lambda_{J}=f_{I J}{ }^{K} \lambda_{K}, \quad £_{I} \lambda_{\alpha}=0,
$$

but $W_{3}, \lambda_{I}, \lambda_{\alpha}$ are otherwise arbitrary. It is easily checked that the new $E_{4}$ is still closed and gauge-invariant. Furthermore, we have checked that the integrals $\int_{M_{10-d}} E_{4}^{3}$ and $\int_{M_{10-d}} E_{4} X_{8}$ are invariant under the deformation (3.21), which implies that the inflow anomaly polynomial is unaffected by it. In the language of $G$-equivariant cohomology, the deformation (3.21) corresponds to adding $G$-equivariantly exact pieces to $E_{4}$, which does not change the $G$-equivariant cohomology class of $E_{4}$.

A more interesting class of ambiguities in the determination of $E_{4}$ is the following. For a given $V_{4}$, we can construct equivariant completions $V_{4}^{\text {eq }}$ that correspond to different $G$-equivariant cohomology classes. More explicitly, we can consider the modification

$$
\omega_{I} \rightarrow \omega_{I}+c_{I}^{\alpha} \omega_{\alpha}, \quad \sigma_{I J} \rightarrow \sigma_{I J}+c_{(I}^{\alpha} \sigma_{J) \alpha}+u_{I J} .
$$

The quantities $c_{I}^{\alpha}, u_{I J}$ are constants. Compatibility with (3.10) requires

$$
f_{I J}{ }^{K} c_{K}^{\alpha}=0, \quad f_{I J_{1}}{ }^{K} u_{K J_{2}}+f_{I J_{2}}{ }^{K} u_{J_{1} K}=0 .
$$

In other words, $c_{I}^{\alpha}$ must be acted upon trivially by the adjoint representation, which means that $c_{I}^{\alpha}$ can be non-zero only if $I$ labels a generator of an Abelian factor of the isometry 
group. On the other hand, the constants $u_{I J}$ must be components of a symmetric invariant tensor in the adjoint representation of the isometry algebra. For instance, if the isometry group is simple, $u_{I J}$ must be a multiple of the Cartan-Killing form.

Contrary to (3.21), the modification (3.23) changes the class defined of $V_{4}^{\mathrm{eq}}$, and hence $E_{4}$. It should be noted, however, that the effect of the shift parametrized by the constants $c_{I}^{\alpha}$ can always be undone by a linear redefinition of the external curvatures $F^{I}, F^{\alpha}$,

$$
F^{I} \rightarrow F^{I}, \quad \frac{1}{2 \pi} F^{\alpha} \rightarrow \frac{1}{2 \pi} F^{\alpha}-c_{I}^{\alpha} F^{I} .
$$

In this sense, the only genuine ambiguity in (3.23) is the part parametrized by the constants $u_{I J}$. We notice that any shift of $\sigma_{I J}$ with $u_{I J}$ generates closed and gauge-invariant terms with purely external legs, which can always be reabsorbed in the 4 -form $\gamma_{4}$. The (in)dependence of the inflow anomaly polynomial on $\gamma_{4}$ is studied in appendix C.

In a completely similar way, we can choose inequivalent equivariant completions of $\omega_{\alpha}$. Pragmatically, we consider the shifts

$$
\sigma_{I \alpha} \rightarrow \sigma_{I \alpha}+u_{I \alpha}
$$

where the constants $u_{I \alpha}$ are constrained by (3.14),

$$
£_{I} u_{J \alpha}=f_{I J}{ }^{K} u_{K \alpha} .
$$

Once again, a shift parametrized by $u_{I \alpha}$ corresponds to a modification of $\gamma_{4}$.

We close this section by highlighting an important feature of the case $d=4$, which is explained in more detail in appendix C.2. For $d=4$, one of the vectors $A^{\alpha}$ originating from expansion of $C_{4}$ onto a basis $\omega_{\alpha}$ of harmonic 2-form is massive. This linear combination does not correspond to a symmetry of the system. The associated background curvature must then be set to zero. More precisely, this holds provided we choose the 2 -forms $\omega_{I}$ in such a way that

$$
\int_{M_{6}} V_{4} \omega_{I}=0
$$

This condition can always be achieved by shifting $\omega_{I}$ by an appropriate linear combination of $\omega_{\alpha}$ 's. If (3.28) holds, the linear combination of $F^{\alpha}$ curvatures that must be set to zero is

$$
N_{\alpha} F^{\alpha}=0, \quad N_{\alpha}=\int_{M_{6}} V_{4} \omega_{\alpha} .
$$

The constraint $N_{\alpha} F^{\alpha}=0$ is essential in order to get the correct anomaly in four dimensions. It plays a particularly non-trivial role in the example studied in section 5.2.

\section{Examples in six dimensions}

In this section we exemplify the method of section 3.1 for the construction of $E_{4}$ in two examples involving a stack of $N$ M5-branes in six uncompactified dimensions. In the first example, the branes sit at a smooth point in the transverse directions, while in the second example they probe an orbifold singularity. 


\subsection{A stack of $N$ M5-branes}

Anomaly inflow for this setup is well-known $[18,19]$. The analysis of this section is useful for the rest of this paper, since it allows us to introduce the objects (4.5) which are later used for various applications.

The 4-form $V_{4}$, which encodes the background $G_{4}$-flux configuration, must be proportional to the volume form on the internal space $M_{4}=S^{4}$. We write

$$
V_{4}=N \operatorname{vol}_{S^{4}} .
$$

In our normalization, $\int_{S^{4}} \operatorname{vol}_{S^{4}}=1$. The constant $N$ is an integer by virtue of $G_{4}$-flux quantization in M-theory, and counts the number of M5-branes in the stack.

The internal space $S^{4}$ admits an $\mathrm{SO}(5)$ isometry group. Let us introduce constrained coordinates $y^{A}, A=1, \ldots, 5$ with $y^{A} y_{A}=1$. The gauging of the $\mathrm{SO}(5)$ isometry is given by

$$
\left(d y^{A}\right)^{\mathrm{g}}=d y^{A}-A^{A B} y_{B},
$$

where $A^{[A B]}$ is the external $\mathrm{SO}(5)$ connection, with field strength

$$
F^{A B}=d A^{A B}-A^{A C} A_{C}^{B} .
$$

Notice that $S^{4}$ does not admit any non-trivial harmonic 2-form. As a result, we do not have any additional external connections.

The 4 -form $E_{4}$ is determined by solving (3.10), (3.12). The result of the analysis takes the form

$$
E_{4}=\bar{V}_{4}+F^{A B} \bar{\omega}_{A B}^{\mathrm{g}}+F^{A B} F^{C D} \bar{\sigma}_{A B, C D}+\gamma_{4},
$$

where $\gamma_{4}$ is an arbitrary closed, gauge-invariant 4 -form with external legs, and we have defined the following forms on $S^{4}$,

$$
\begin{aligned}
\bar{V}_{4} & =\frac{3 N}{8 \pi^{2}} \cdot \frac{1}{4 !} \epsilon_{A_{1} \ldots A_{5}} d y^{A_{1}} d y^{A_{2}} d y^{A_{3}} d y^{A_{4}} y^{A_{5}}, \\
\bar{\omega}_{A B} & =\frac{3 N}{8 \pi^{2}} \cdot \frac{-2}{4 !} \epsilon_{A B C_{1} C_{2} C_{3}} d y^{C_{1}} d y^{C_{2}} y^{C_{3}}, \\
\bar{\sigma}_{A B, C D} & =\frac{3 N}{8 \pi^{2}} \cdot \frac{1}{4 !} \epsilon_{A B C D E} y^{E} .
\end{aligned}
$$

If we set $\gamma_{4}=0$, the 4 -form $E_{4}$ in (4.4) is proportional to the global angular form of $\mathrm{SO}(5)$, which appears in the original analysis of $[18,19]$.

The Pontryagin classes of $T M_{11}$ can be computed exploiting the decomposition of the $11 \mathrm{~d}$ tangent bundle restricted on the worldvolume $W_{6}$ of the brane,

$$
T M_{11} \rightarrow T W_{6} \oplus N_{\mathrm{SO}(5)},
$$

where $N_{\mathrm{SO}(5)}$ is the bundle encoding the $\mathrm{SO}(5)$ gauging. We have

$$
\begin{aligned}
& p_{1}\left(T M_{11}\right)=p_{1}\left(T W_{6}\right)+p_{1}(\mathrm{SO}(5)), \\
& p_{2}\left(T M_{11}\right)=p_{2}\left(T W_{6}\right)+p_{2}(\mathrm{SO}(5))+p_{1}\left(T W_{6}\right) p_{1}(\mathrm{SO}(5)),
\end{aligned}
$$


and hence

$$
X_{8}=-\frac{1}{48}\left[p_{2}\left(T W_{6}\right)+p_{2}(\mathrm{SO}(5))\right]+\frac{1}{192}\left[p_{1}\left(T W_{6}\right)-p_{1}(\mathrm{SO}(5))\right]^{2} .
$$

Notice that $X_{8}$ has no legs along the internal $S^{4}$ directions.

We can now compute the inflow anomaly polynomial, using (4.4) and (4.8). The result reads

$$
\begin{aligned}
I_{8}^{\text {inflow }}= & -\frac{1}{24} N^{3} p_{2}(\mathrm{SO}(5))-\frac{1}{2} N \gamma_{4}^{2} \\
& +\frac{1}{48} N\left[p_{2}\left(T W_{6}\right)+p_{2}(\mathrm{SO}(5))\right]-\frac{1}{192} N\left[p_{1}\left(T W_{6}\right)-p_{1}(\mathrm{SO}(5))\right]^{2} .
\end{aligned}
$$

The first line collects the contribution of the $E_{4}^{3}$ term, while the second line contains the contribution of the $E_{4} X_{8}$ term. To verify (4.9) we can make use of the identities (A.17), (A.18). In our conventions,

$$
p_{1}(\mathrm{SO}(5))=-\frac{1}{2} \frac{1}{(2 \pi)^{2}} \operatorname{tr} F^{2}, \quad p_{2}(\mathrm{SO}(5))=\frac{1}{8} \frac{1}{(2 \pi)^{2}}\left[\left(\operatorname{tr} F^{2}\right)^{2}-2 \operatorname{tr} F^{4}\right]
$$

where the trace is in the fundamental representation of $\mathrm{SO}(5)$.

In appendix $\mathrm{C}$ we argue that $\gamma_{4}$ is fixed by extremizing $I_{8}^{\text {inflow }}$ with respect to an arbitrary variation in $\gamma_{4}$. In the present situation, we obtain simply

$$
\gamma_{4}=0
$$

For this value of $\gamma_{4}$, the result (4.9) agrees with the original inflow polynomial of [19].

\subsection{M5-branes probing an ADE singularity}

Let us now analyze a setup in which a stack of M5-branes probes a $\mathbb{C}^{2} / \Gamma$ singularity, where $\Gamma$ is an ADE subgroup of $\mathrm{SU}(2)[3,30-34]$. The probe picture in the UV is as follows.

Let us consider the transverse $\mathbb{R}^{5}$ to the M5-branes, with coordinates $y^{1}, \ldots, y^{5}$. The group $\Gamma$ acts on $\mathbb{R}^{5}$ by leaving $y^{5}$ invariant, and acting on $\mathbb{R}^{4}$ parametrized by $y^{1}, \ldots, y^{4}$. More precisely, the action of $\Gamma$ is embedded in the factor $\mathrm{SU}(2)_{L}$ of the isometry group $\mathrm{SO}(4) \cong \mathrm{SU}(2)_{L} \times \mathrm{SU}(2)_{R}$ of the $\mathbb{R}^{4}$ spanned by $y^{1,2,3,4}$. All points on the $y^{5}$ axis are fixed points under the action of $\Gamma$. In the probe picture, the stack is positioned at the origin $y^{1,2,3,4,5}=0$. Because of the $\Gamma$ quotient, supersymmetry is reduced from $(2,0)$ to $(1,0)$.

Before performing the quotient, the stack is surrounded by the round sphere $S^{4} \subset \mathbb{R}^{5}$. After acting with $\Gamma, S^{4}$ is replaced with $S^{4} / \Gamma$. The north and south poles of $S^{4}$, located at $y^{5}= \pm 1, y^{1,2,3,4}=0$, are both fixed points for the $\Gamma$ action. Locally near each pole we have an orbifold singularity $\mathbb{C}^{2} / \Gamma$. The orbifold singularities at each pole can be resolved preserving $6 \mathrm{~d}(1,0)$ supersymmetry. If $\mathfrak{g}_{\Gamma}$ is the ADE Lie algebra associated to $\Gamma$, we write $r_{\Gamma}=\operatorname{rank}\left(\mathfrak{g}_{\Gamma}\right)$. The resolution introduces a number $r_{\Gamma}$ of $\mathbb{C P}^{1}$ curves, whose intersection pattern reproduces the Dynkin diagram of $\mathfrak{g}_{\Gamma}$. We use the symbol $M_{4}$ for the smooth space obtained from $S^{4} / \Gamma$ by resolving the singularities at the north and south poles.

The isometry group $\mathrm{SO}(5)$ of $S^{4}$ is reduced by the action of $\Gamma$. More precisely, the isometry group of $S^{4} / \Gamma$ is the subgroup of $\mathrm{SU}(2)_{L} \times \mathrm{SU}(2)_{R}$ that commutes with the action 
of $\Gamma$. If $\Gamma=\mathbb{Z}_{k}$ with $k \geq 3$, the isometry group is $\mathrm{U}(1)_{L} \times \mathrm{SU}(2)_{R}$, with $\mathrm{U}(1)_{L}$ the Cartan of $\mathrm{SU}(2)_{L}$. If $k=1,2$ the isometry group is the full $\mathrm{SU}(2)_{L} \times \mathrm{SU}(2)_{R}$. Finally, if $\Gamma$ is of type $\mathrm{D}, \mathrm{E}$, the isometry group is $\mathrm{SU}(2)_{R}$ only.

To treat all cases uniformly, we formally introduce external connections for the full $\mathrm{SU}(2)_{L} \times \mathrm{SU}(2)_{R}$. It is understood that the external $\mathrm{SU}(2)_{L}$ connection is zero for $\Gamma$ of $\mathrm{D}$, E type, it is along the Cartan for $\Gamma=\mathbb{Z}_{k}, k \geq 3$, and it is a full non-Abelian connection for $k=1,2$.

The resolved space $M_{4}$ admits non-trivial 2-cycles, and hence harmonic 2-forms. They are dual to the resolution $\mathbb{C P}^{1}$ 's at the north and south poles. We write these harmonic 2 -forms as $\omega_{\mathrm{N} i}, \omega_{\mathrm{S} i}$, with the index $i=1, \ldots, r_{\Gamma}$ labelling the Cartan generators of the ADE Lie algebra $\mathfrak{g}_{\Gamma}$. These harmonic 2 -forms correspond to additional flavor symmetries of the setup. In the resolved phase, the flavor symmetry associated to the harmonic 2-forms is $\left(\mathrm{U}(1)^{r_{\Gamma}}\right)_{\mathrm{N}} \times\left(\mathrm{U}(1)^{r_{\Gamma}}\right)_{\mathrm{S}}$. If we shrink the resolution cycles to zero size, we have enhancement to the non-Abelian symmetry $\left(G_{\Gamma}\right)_{\mathrm{N}} \times\left(G_{\Gamma}\right)_{\mathrm{S}}$, where $G_{\Gamma}$ is the Lie group associated to $\Gamma$.

We are now in a position to discuss the 4 -form $E_{4}$ for the setup under examination. It can be written as

$$
E_{4}=\bar{V}_{4}^{\mathrm{g}}+F^{A B} \bar{\omega}_{A B}^{\mathrm{g}}+F^{A B} F^{C D} \bar{\sigma}_{A B, C D}+\frac{F^{\mathrm{N} i}}{2 \pi} \omega_{\mathrm{N} i}+\frac{F^{\mathrm{S} i}}{2 \pi} \omega_{\mathrm{S} i}+\gamma_{4} .
$$

Several comments are in order. The above expression is written in terms of the $\mathrm{SO}(5)$ curvature $F^{A B}$. It is understood, however, that $F^{A B}$ is only non-zero along the generators of the subgroup $\mathrm{SU}(2)_{L} \times \mathrm{SU}(2)_{R} \subset \mathrm{SO}(5)$. The quantities $\bar{V}_{4}, \bar{\omega}_{A B}, \bar{\sigma}_{A B, C D}$ are as in (4.5), but with the replacement $N \rightarrow N|\Gamma|$, where $|\Gamma|$ is the order of the finite group $\Gamma$. The extra factor $|\Gamma|$ is needed to compensate for the fact that the $\Gamma$ action introduces a factor $1 /|\Gamma|$ in all integrals over $S^{4} / \Gamma$. The curvatures $F^{\mathrm{N} i}, F^{\mathrm{S} i}$ are associated to the flavor symmetry at the poles in the resolved phase. We stress that the harmonic 2-forms $\omega_{\mathrm{N} i}, \omega_{\mathrm{S} i}$ are invariant under the isometry group of $S^{4} / \Gamma$, because they are localized at the poles, which are fixed under $\mathrm{SU}(2)_{L} \times \mathrm{SU}(2)_{R}$. This is why we do not have to gauge $\omega_{\mathrm{N} i}, \omega_{\mathrm{S} i}$ in (4.12). Finally, $\gamma_{4}$ is an arbitrary closed, gauge-invariant, external 4 -form.

The derivation of the inflow anomaly polynomial for this setup was discussed in [24], without the $\gamma_{4}$ term. We review the derivation, including $\gamma_{4}$, in appendix D. The result reads

$$
\begin{aligned}
-I_{8}^{\text {inflow }}= & \frac{N^{3}|\Gamma|^{2}}{24}\left[c_{2}(L)-c_{2}(R)\right]^{2}+\frac{1}{2} N \gamma_{4}^{2}+\frac{1}{4} \gamma_{4}\left[\frac{\operatorname{tr}\left(F^{\mathrm{N}}\right)^{2}}{(2 \pi)^{2}}-\frac{\operatorname{tr}\left(F^{\mathrm{S}}\right)^{2}}{(2 \pi)^{2}}\right] \\
& +\frac{N|\Gamma|}{8}\left[c_{2}(L)-c_{2}(R)\right]\left[\frac{\operatorname{tr}\left(F^{\mathrm{N}}\right)^{2}}{(2 \pi)^{2}}+\frac{\operatorname{tr}\left(F^{\mathrm{S}}\right)^{2}}{(2 \pi)^{2}}\right] \\
& +\frac{N|\Gamma| \chi_{\Gamma}}{48}\left[c_{2}(L)-c_{2}(R)\right]\left[p_{1}\left(T W_{6}\right)+4 c_{2}(R)\right]+\frac{N}{48} c_{2}(L)\left[p_{1}\left(T W_{6}\right)+4 c_{2}(R)\right] \\
& +\frac{N}{192}\left[p_{1}\left(T W_{6}\right)^{2}-4 p_{2}\left(T W_{6}\right)\right]+\frac{N}{48} c_{2}(R) p_{1}\left(T W_{6}\right)
\end{aligned}
$$

The quantities $c_{2}(L, R)$ are the second Chern classes of $\mathrm{SU}(2)_{L, R}$, while $p_{1,2}\left(T W_{6}\right)$ are the Pontryagin classes of the external $6 \mathrm{~d}$ background metric. We have written the result in terms of the full non-Abelian flavor symmetry curvatures $F^{\mathrm{N}}, F^{\mathrm{S}}$, even though only the 


\begin{tabular}{|r|c|c|c|c|c|}
\hline Lie algebra $\mathfrak{g}_{\Gamma}$ & $\mathrm{SU}(k)$ & $\mathrm{SO}(2 k)$ & $E_{6}$ & $E_{7}$ & $E_{8}$ \\
\hline $\operatorname{rank} r_{\Gamma}$ & $k-1$ & $k$ & 6 & 7 & 8 \\
\hline $\operatorname{order}|\Gamma|$ & $k$ & $4 k-8$ & 24 & 48 & 120 \\
\hline
\end{tabular}

Table 1. The rank $r_{\Gamma}$ and order $|\Gamma|$ for $\operatorname{ADE}$ subgroups $\Gamma$ of $\mathrm{SU}(2)$.

Cartan curvatures $F^{\mathrm{N} i}, F^{\mathrm{S} i}$ are directly accessible in the supergravity approximation. The quantity $\chi_{\Gamma}$ is the Euler characteristic of the ALE space that resolves the $\mathbb{C}^{2} / \Gamma$ orbifold. It is given by

$$
\chi_{\Gamma}=r_{\Gamma}+1-\frac{1}{|\Gamma|} .
$$

The ranks $r_{\Gamma}$ and orders $|\Gamma|$ for all ADE groups are summarized in table 1.

According to the general discussion of appendix C.1, the external 4 -form $\gamma_{4}$ is fixed by extremizing $I_{8}^{\text {inflow }}$, which is equivalent to imposing the tadpole cancellation condition in M-theory. In the present situation, we obtain

$$
\gamma_{4}=-\frac{1}{4 N}\left[\frac{\operatorname{tr}\left(F^{\mathrm{N}}\right)^{2}}{(2 \pi)^{2}}-\frac{\operatorname{tr}\left(F^{\mathrm{S}}\right)^{2}}{(2 \pi)^{2}}\right] .
$$

Plugging this back into (4.13), we obtain

$$
\begin{aligned}
-I_{8}^{\text {inflow }}= & \frac{N^{3}|\Gamma|^{2}}{24}\left[c_{2}(L)-c_{2}(R)\right]^{2}-\frac{1}{32 N}\left[\frac{\operatorname{tr}\left(F^{\mathrm{N}}\right)^{2}}{(2 \pi)^{2}}-\frac{\operatorname{tr}\left(F^{\mathrm{S}}\right)^{2}}{(2 \pi)^{2}}\right]^{2} \\
& +\frac{N|\Gamma|}{8}\left[c_{2}(L)-c_{2}(R)\right]\left[\frac{\operatorname{tr}\left(F^{\mathrm{N}}\right)^{2}}{(2 \pi)^{2}}+\frac{\operatorname{tr}\left(F^{\mathrm{S}}\right)^{2}}{(2 \pi)^{2}}\right] \\
& +\frac{N|\Gamma| \chi_{\Gamma}}{48}\left[c_{2}(L)-c_{2}(R)\right]\left[p_{1}\left(T W_{6}\right)+4 c_{2}(R)\right]+\frac{N}{48} c_{2}(L)\left[p_{1}\left(T W_{6}\right)+4 c_{2}(R)\right] \\
& +\frac{N}{192}\left[p_{1}\left(T W_{6}\right)^{2}-4 p_{2}\left(T W_{6}\right)\right]+\frac{N}{48} c_{2}(R) p_{1}\left(T W_{6}\right) .
\end{aligned}
$$

This result agrees with the analysis of [24]. It is interesting to point out that, in the computation of [24], the term $-\frac{1}{32 N}(2 \pi)^{-2}\left[\operatorname{tr}\left(F^{\mathrm{N}}\right)^{2}-\operatorname{tr}\left(F^{\mathrm{S}}\right)^{2}\right]^{2}$ is interpreted as a GreenSchwarz term associated to the center of mass mode of the M5-brane stack, and is included by hand. In our derivation, it is automatically generated by $\gamma_{4}$-extremization.

Let us consider the case $\Gamma=\mathbb{Z}_{k}$. Using the full anomaly polynomial recorded in [25] for the interacting $6 \mathrm{~d}(1,0) \mathrm{SCFT}$, we can extract the contribution of decoupling modes related to the center of mass of the M5-brane stack. To compare with [25], we replace

$$
c_{2}(L) \rightarrow-c_{1}(s)^{2},
$$

where we are using the notation of [25] for the first Chern class $c_{1}(s)$ of the Cartan $\mathrm{U}(1)_{L}$ of $\mathrm{SU}(2)_{L}$. Comparing (4.16) to the results of [25], we infer

$$
\begin{aligned}
I_{8}^{\text {decoupl }} & =-I_{8}^{\text {inflow }}-I_{8}^{\mathrm{SCFT}} \\
& =I_{8}^{\text {tensor }}+\frac{1}{2} I_{8}^{\mathrm{vec}, \mathrm{N}}+\frac{1}{2} I_{8}^{\mathrm{vec}, \mathrm{S}}-\frac{1}{6} k c_{1}(s)\left[\frac{\operatorname{Tr}_{\text {fund }}\left(F^{\mathrm{N}}\right)^{3}}{(2 \pi)^{3}}-\frac{\operatorname{Tr}_{\text {fund }}\left(F^{\mathrm{S}}\right)^{3}}{(2 \pi)^{3}}\right] .
\end{aligned}
$$


The quantities $I_{8}^{\text {tensor }}, I_{8}^{\mathrm{vec}, \mathrm{N}}$, are given by ${ }^{6}$

$$
\begin{aligned}
I_{8}^{\mathrm{vec}, \mathrm{N}}= & -\frac{k^{2}-1}{24} c_{2}(R)^{2}-\frac{k^{2}-1}{48} c_{2}(R) p_{1}\left(T W_{6}\right)-\frac{k^{2}-1}{5760}\left[7 p_{1}\left(T W_{6}\right)^{2}-4 p_{2}\left(T W_{6}\right)\right] \\
& -\frac{k}{4} c_{2}(R) \frac{\operatorname{tr}\left(F^{N}\right)^{2}}{(2 \pi)^{2}}-\frac{k}{48} p_{1}\left(T W_{6}\right) \frac{\operatorname{tr}\left(F^{N}\right)^{2}}{(2 \pi)^{2}}-\frac{1}{16}\left[\frac{\operatorname{tr}\left(F^{N}\right)^{2}}{(2 \pi)^{2}}\right]^{2}-\frac{k}{12} \frac{\operatorname{Tr}_{\text {fund }}\left(F^{N}\right)^{4}}{(2 \pi)^{4}}, \\
I_{8}^{\mathrm{tensor}}= & \frac{1}{24} c_{2}(R)^{2}+\frac{1}{48} c_{2}(R) p_{1}\left(T W_{6}\right)+\frac{23}{5760} p_{1}\left(T W_{6}\right)^{2}-\frac{29}{1440} p_{2}\left(T W_{6}\right) .
\end{aligned}
$$

The quantity $I_{8}^{\mathrm{vec}, \mathrm{S}}$ is completely analogous to $I_{8}^{\mathrm{vec}, \mathrm{N}}$ given above.

\section{$5 \quad$ Examples in four dimensions}

In this section we examine two $4 \mathrm{~d}$ setups to exemplify our prescription for the computation of $I_{6}^{\text {inflow }}$. In a first class of examples, the space $M_{6}$ is an $S^{4}$ fibration over a smooth Riemann surface. This case corresponds to the setups analyzed in BBBW [9, 10]. Next, we analyze the geometry $M_{6}$ that is read off from the GMSW [20] $A d S_{5}$ solution to $11 \mathrm{~d}$ supergravity.

\section{1 $S^{4}$ fibrations over a smooth Riemann surface}

Let us consider a stack of M5-branes wrapping a genus- $g$ Riemann surface without punctures $\Sigma_{g}$. In this setup, the internal space $M_{6}$ is an $S^{4}$ fibration over $\Sigma_{g}$. Upon including external connections, $M_{6}$ is fibered over external spacetime $W_{4}$. The relevant fibrations are thus

$$
M_{6} \hookrightarrow M_{10} \rightarrow W_{4}, \quad S^{4} \hookrightarrow M_{6} \rightarrow \Sigma_{g} .
$$

In order to implement anomaly inflow, we need to study the topology and isometries of $M_{6}$.

\subsubsection{Topology and isometries of $M_{6}$}

In this work we study a class of fibrations $S^{4} \hookrightarrow M_{6} \rightarrow \Sigma_{g}$ that preserve $4 \mathrm{~d} \mathcal{N}=1$ supersymmetry $[9,10]$. In terms of the ambient space $\mathbb{R}^{5} \supset S^{4}$, we refer to the decomposition $\mathbb{R}^{5}=\mathbb{C}_{1} \times \mathbb{C}_{2} \times \mathbb{R}$. The topology of $M_{6}$ is then encoded in the two line bundles $\mathcal{L}_{1}, \mathcal{L}_{2}$ that describe the twisting of the two $\mathbb{C}_{1}, \mathbb{C}_{2}$ factors on the Riemann surface. Let $q_{1}, q_{2}$ be the degrees of the line bundles. In order to preserve supersymmetry, the total space $\mathcal{L}_{1} \oplus \mathcal{L}_{2} \rightarrow \Sigma_{g}$ has to be a Calabi-Yau threefold, which amounts to the requirement

$$
q_{1}+q_{2}=-\chi\left(\Sigma_{g}\right)=2(g-1) .
$$

Setups in which $g=1$, i.e. the Riemann surface is a torus, require special care, because of the presence of emergent symmetries. For this reason, we restrict ourselves to the cases

\footnotetext{
${ }^{6}$ Following [25], the traces of $F^{N}$ are defined in such a way that

$$
\frac{\operatorname{tr}\left(F^{\mathrm{N}}\right)^{2}}{(2 \pi)^{2}}=-2 \sum_{i}\left(n_{i}^{\mathrm{N}}\right)^{2}, \quad \frac{\operatorname{Tr}_{\text {fund }}\left(F^{\mathrm{N}}\right)^{3}}{(2 \pi)^{3}}=\sum_{i}\left(n_{i}^{\mathrm{N}}\right)^{3}, \quad \frac{\operatorname{Tr}_{\text {fund }}\left(F^{\mathrm{N}}\right)^{4}}{(2 \pi)^{4}}=\sum_{i}\left(n_{i}^{\mathrm{N}}\right)^{4}
$$

where $n_{i}^{\mathrm{N}}$ are the Chern roots of $\mathrm{SU}(k)_{\mathrm{N}}, i=1, \ldots, k$ and $\sum_{i} n_{i}^{\mathrm{N}}=0$. The same conventions hold for $\mathrm{SU}(k)_{\mathrm{S}}$.
} 
of a higher-genus Riemann surface, $g \geq 2$, or a sphere, $g=0$. In our discussion $q_{1}$ and $q_{2}$ cannot therefore be simultaneously zero. If $q_{1}=0$ or $q_{2}=0$, supersymmetry enhances to $\mathcal{N}=2$.

The topology of $M_{6}$ can be equivalently described in terms of the background value of a non-zero background value for the $\mathrm{SO}(5)$ field strength $F^{A B}$, which is proportional to the volume form on $\Sigma_{g}$. For the setups under examination, the background $F^{A B}$ takes the form

$$
F_{\Sigma}^{A B}=q^{A B} V_{\Sigma}, \quad q^{A B}=\left(\begin{array}{ccccc}
0 & q_{1} & & \\
-q_{1} & 0 & & \\
& & 0 & q_{2} \\
& -q_{2} & 0 & \\
& & & & 0
\end{array}\right)
$$

The subscript $\Sigma$ on $F$ is a reminder that this is the background part, or twist part, of the $\mathrm{SO}(5)$ field strength, as opposed to the external $4 \mathrm{~d}$ gauge part. The 2 -form $V_{\Sigma}$ is proportional to the volume form on $\Sigma_{g}$, and is normalized according to

$$
\int_{\Sigma_{g}} V_{\Sigma}=2 \pi
$$

In order to apply the recipe of section 3.1 for the construction of $E_{4}$, we need to identify the isometries of $M_{6}$ that we intend to couple to $4 \mathrm{~d}$ gauge fields. We have two distinct classes of isometries:

(i) For any genus $g$, a subgroup $\mathrm{SO}(2)_{1} \times \mathrm{SO}(2)_{2}$ of the $\mathrm{SO}(5)$ isometry group of the $S^{4}$ fiber is preserved by the twist described by (5.3). We therefore introduce two Abelian external connections $A^{1}, A^{2}$, with field strengths $F^{1}=d A^{1}, F^{2}=d A^{2}$, to gauge this residual isometry. The embedding of $A^{1}, A^{2}$ into the full $\mathrm{SO}(5)$ connection, and the analogous relation for the field strengths, read

$$
A_{\mathrm{ext}}^{A B}=\left(\begin{array}{ccccc}
0 & -A^{1} & & & \\
A^{1} & 0 & & & \\
& & & -A^{2} & \\
& & A^{2} & 0 & \\
& & & & 0
\end{array}\right), \quad F_{\mathrm{ext}}^{A B}=\left(\begin{array}{ccccc}
0 & -F^{1} & & \\
F^{1} & 0 & & & \\
& & & -F^{2} \\
& & F^{2} & 0 & \\
& & & & 0
\end{array}\right),
$$

with the subscript "ext" standing for external. If $q_{2}=0$, the $\mathrm{SO}(2)_{2}$ factor enhances to $\mathrm{SO}(3)_{2}$, and $F^{2}$ is replaced by the suitable non-Abelian $\mathrm{SO}(3)_{2}$ field strength. Similar remarks apply if $q_{1}=0$.

(ii) In the special case $g=0, M_{6}$ possesses additional isometries that originate from the isometry group $\mathrm{SO}(3)_{S^{2}}$ of the Riemann surface, which is a 2-sphere endowed with its standard round metric. As explained in appendix E, the Killing vectors of the base $\Sigma_{g=0}=S^{2}$ considered in isolation extend to bona fide Killing vectors of the entire space $M_{6}$, for any value of $q_{1}, q_{2}$. We use the notation $A^{a}$, with $a=1,2,3$, for the external $\mathrm{SO}(3)_{S^{2}}$ connection that gauges this additional isometry, and $F^{a}$ for the corresponding field strength. 


\subsubsection{Aside on terminology: twisting vs gauging}

The nested fibration structure (5.1) of the setups under examination allows us to define two distinct operations on differential forms, which we refer to as twisting and gauging.

The twisting operation is defined with reference to the fibration $S^{4} \hookrightarrow M_{6} \rightarrow \Sigma_{g}$. It makes use of the internal field strength (5.3), but it does not involve any external $4 \mathrm{~d}$ gauge connection. Twisting affects forms on $M_{6}$ with legs along the $S^{4}$ fiber directions, while it has no effect on legs along the Riemann surface. Operationally, in terms of the constrained coordinates $y^{A} y_{A}=1$ of the $S^{4}$ fibers, the twisting operation amounts to the replacement

$$
d y^{A} \rightarrow\left(d y^{A}\right)^{\mathrm{t}}=d y^{A}-q^{A B} y_{B} A_{\Sigma}, \quad d A_{\Sigma}=V_{\Sigma} .
$$

The 1-form $A_{\Sigma}$ on the Riemann surface is an antiderivative of the volume form and is only locally defined. Because of the non-trivial fibration, the untwisted 1-forms $d y^{A}$ are not well-defined on $M_{6}$. Their twisted counterparts $\left(d y^{A}\right)^{\mathrm{t}}$, however, are good objects in $M_{6}$.

Let us now turn to the gauging operation. This is the same operation discussed in section 3.1, and is based on the isometries of $M_{6}$. Let us first consider a higher-genus Riemann surface. The only isometries are then of the class (i) above. Since isometries of class (i) originate from the $S^{4}$ fiber of $M_{6}$, the gauging procedure has no effect on $V_{\Sigma}$. We can write

$$
\text { higher-genus } \Sigma_{g}: \quad\left\{\begin{aligned}
\left(d y^{A}\right)^{\mathrm{t}} & \rightarrow\left(d y^{A}\right)^{\mathrm{tg}}=d y^{A}-q^{A B} y_{B} A_{\Sigma}-A_{\mathrm{ext}}^{A B} y_{B} \\
V_{\Sigma} & \rightarrow V_{\Sigma}^{\mathrm{g}}=V_{\Sigma}
\end{aligned}\right.
$$

Notice that, since the untwisted $d y^{A} 1$-forms are not well-defined on $M_{6}$, it does not make sense to consider $\left(d y^{A}\right)^{\mathrm{g}}$.

If the Riemann surface is a sphere, we have both isometries of class (i) and of class (ii). To proceed, it is convenient to describe the 2 -sphere by means of three constrained coordinates $z^{a}, a=1,2,3$, satisfying $z^{a} z_{a}=1$. The gauging operation in this case satisfies

$$
\text { two-sphere : } \quad\left\{\begin{aligned}
\left(d y^{A}\right)^{\mathrm{t}} & \rightarrow\left(d y^{A}\right)^{\mathrm{tg}}=d y^{A}-q^{A B} y_{B} A_{\Sigma}-A_{\mathrm{ext}}^{A B} y_{B}+\frac{1}{2} q^{A B} y_{B} z_{a} A^{a}, \\
d z^{a} & \rightarrow\left(d z^{a}\right)^{\mathrm{g}}=d z^{a}+\epsilon^{a b c} A_{b} z_{c}, \\
V_{\Sigma} & \rightarrow V_{\Sigma}^{\mathrm{g}}=\frac{1}{4} \epsilon_{a b c}\left(d z^{a}\right)^{\mathrm{g}}\left(d z^{b}\right)^{\mathrm{g}} z^{c} .
\end{aligned}\right.
$$

Crucially, gauging of the additional $\mathrm{SO}(3)_{S^{2}}$ isometry of class (ii) involves legs along the Riemann surface. In appendix E we collect some useful formulae that are helpful in checking the above relations.

\subsubsection{Construction of $E_{4}$}

The first task in the construction of $E_{4}$ is the identification of the 4-form $V_{4}$, to which $E_{4}$ reduces if we turn off all external $4 \mathrm{~d}$ connections. We claim that

$$
V_{4}=\bar{V}_{4}^{\mathrm{t}}+q^{A B} V_{\Sigma} \bar{\omega}_{A B}^{\mathrm{t}}
$$

The forms $\bar{V}_{4}$ and $\bar{\omega}_{A B}$ have legs on the $S^{4}$ fibers and were introduced in (4.5). The subscript " $t$ " signals the twisting operation discussed in the previous subsection. The untwisted 
4-form $\bar{V}_{4}$ is closed, but it is not well-defined in the total internal space $M_{6}$. Its twisted counterpart $\bar{V}_{4}^{\mathrm{t}}$ is a good object in $M_{6}$, but it is not closed. This explains the necessity of the other term in (5.8). Indeed, to see that $V_{4}$ is closed, we simply observe that it is proportional to the global angular form of $\mathrm{SO}(5)$, provided we replace the $\mathrm{SO}(5)$ field strength $F^{A B}$ with $F_{\Sigma}^{A B}$ as in (5.3). (The term with two $F_{\Sigma}^{A B}$ factors is then zero because $V_{\Sigma} V_{\Sigma}=0$.)

We claim that $V_{4}$ can be taken to be as in (5.8) without any loss of generality. This can be motivated as follows. Firstly, we know from section 3.2 that any modification of $V_{4}$ by an exact 4-form $d W_{3}$ (compatible with the isometries of $M_{6}$ ) does not have any effect on the inflow anomaly polynomial. Secondly, we observe that we do not have any other closed but not exact 4 -form in $M_{6}$.

The space $M_{6}$ admits one non-trivial 4-cycle, given by the $S^{4}$ fiber over a generic point on $\Sigma_{g}$. We then have one harmonic 2-form $\omega$, Poincaré dual to this 4-cycle. We can write

$$
\int_{S^{4}} V_{4}=\int_{M_{6}} V_{4} \omega=N
$$

As discussed in section C.2, one linear combination of the vectors associated to harmonic 2 -forms is massive. Since in this case we only have one harmonic 2-form, its associated vector is massive, and we can simply ignore it in the following discussion. As a result, all external connections are associated to the isometries of class (i) and (ii) discussed above.

We are now in a position to apply the recipe of section 3.1 for the construction of $E_{4}$. We refer the reader to appendix $\mathrm{E}$ for the derivation of $E_{4}$. The result takes the form

$$
E_{4}=\bar{V}_{4}^{\mathrm{tg}}+\mathcal{F}^{A B} \bar{\omega}_{A B}^{\mathrm{tg}}+\mathcal{F}^{A B} \mathcal{F}^{C D} \bar{\sigma}_{A B, C D}+\left(C_{1} F^{1}+C_{2} F^{2}\right)\left(V_{\Sigma}^{\mathrm{g}}-\frac{1}{2} F^{a} z_{a}\right)+\gamma_{4} .
$$

In the previous expression, we have introduced the 2 -forms

$$
\mathcal{F}^{A B}=F_{\mathrm{ext}}^{A B}+q^{A B}\left(V_{\Sigma}^{\mathrm{g}}-\frac{1}{2} F^{a} z_{a}\right) .
$$

The quantities $C_{1,2}$ are constants, while $\gamma_{4}$ is an arbitrary closed, gauge-invariant 4 -form with external legs only.

The values of $C_{1}, C_{2}$ are actually fixed by the following considerations. In section C.2 we derived that one linear combination of the vectors associated to harmonic 2-forms is massive. This results holds under the assumption that a basis of connections is chosen, such that (3.28) holds. In order to check whether $E_{4}$ in (5.10) satisfies (3.28), we need to extract the terms linear in the isometry curvatures $F^{1}, F^{2}$, and $F^{a}$,

$$
E_{4}=V_{4}^{\mathrm{g}}+F^{1} \omega_{1}^{\mathrm{g}}+F^{2} \omega_{2}^{\mathrm{g}}+F^{a} \omega_{a}^{\mathrm{g}}+\ldots
$$

Comparison with (5.10), keeping (5.5) into account, leads to the identifications

$$
\begin{aligned}
& \omega_{1}=-2\left(\bar{\omega}_{12}^{\mathrm{t}}+2 q^{C D} V_{\Sigma} \bar{\sigma}_{12, C D}\right)+C_{1} V_{\Sigma}, \\
& \omega_{2}=-2\left(\bar{\omega}_{34}^{\mathrm{t}}+2 q^{C D} V_{\Sigma} \bar{\sigma}_{34, C D}\right)+C_{2} V_{\Sigma}, \\
& \omega_{a}=-\frac{1}{2} z_{a} q^{A B}\left(\bar{\omega}_{A B}^{\mathrm{t}}+2 q^{C D} V_{\Sigma} \bar{\sigma}_{A B, C D}\right) .
\end{aligned}
$$


Equivalently, the above equations can be read off from (E.29)-(E.33). Making use of the identities (A.17), we verify that $\int_{M_{6}} V_{4} \omega_{a}=0$, while, in order to achieve $\int_{M_{6}} V_{4} \omega_{1,2}=0$, we must set

$$
C_{1}=0, \quad C_{2}=0 .
$$

As a final remark, we would like to point out that, if the Riemann surface is a 2-sphere, the quantity

$$
\frac{V_{\Sigma}^{\mathrm{g}}}{2 \pi}-\frac{1}{2} \frac{F^{a}}{2 \pi} z_{a}
$$

is equal to the global angular form $e_{2}^{S^{2}}$ of $\mathrm{SO}(3)$. The definition and properties of the latter are reviewed in appendix F.

\subsubsection{Computation of $X_{8}$}

To compute $X_{8}$, we adopt the following point of view on the setup under consideration. Let $\widetilde{W}_{6}$ denote the space obtained by combining external spacetime $W_{4}$ with the Riemann surface $\Sigma_{g}$. The Pontryagin classes of $\widetilde{W}_{6}$ detect the curvature of the background metric on $W_{4}$. If the Riemann surface is a sphere, they also detect the gauging of its $\mathrm{SO}(3)_{S^{2}}$ isometries, i.e. the gauging via the connections $A^{a}$. The total space may be thought of as an $S^{4}$ fibration over $\widetilde{W}_{6}$. This fibration is encoded in an $\mathrm{SO}(5)$ bundle. Its connection consists of two parts: one describes the twist of $S^{4}$ over the Riemann surface, the other corresponds to the gauging of the isometries related to the $A_{\text {ext }}^{A B}$ vectors.

The considerations of the previous paragraph lead us to write

$$
\begin{aligned}
& p_{1}\left(T M_{11}\right)=p_{1}\left(T \widetilde{W}_{6}\right)+p_{1}(\mathrm{SO}(5)), \\
& p_{2}\left(T M_{11}\right)=p_{2}\left(T \widetilde{W}_{6}\right)+p_{2}(\mathrm{SO}(5))+p_{1}\left(T \widetilde{W}_{6}\right) p_{1}(\mathrm{SO}(5)) .
\end{aligned}
$$

To proceed, we notice that

$$
p_{1}\left(T \widetilde{W}_{6}\right)=p_{1}\left(T W_{4}\right)+p_{1}\left(\mathrm{SO}(3)_{S^{2}}\right), \quad p_{2}\left(T \widetilde{W}_{6}\right)=0,
$$

with $p_{1}\left(\mathrm{SO}(3)_{S^{2}}\right)$ only present if the Riemann surface is a sphere. It is given by

$$
p_{1}\left(\mathrm{SO}(3)_{S^{2}}\right)=\frac{1}{(2 \pi)^{2}} F_{a} F^{a}
$$

Notice that any form with more than six legs on external spacetime can be discarded.

The final task is the computation of the Pontryagin classes $p_{1,2}(\mathrm{SO}(5))$. They can be written in terms of traces of powers of the $\mathrm{SO}(5)$ field strength,

$$
p_{1}(\mathrm{SO}(5))=-\frac{1}{2} \frac{1}{(2 \pi)^{2}} \operatorname{tr} F_{\mathrm{SO}(5)}^{2}, \quad p_{2}(\mathrm{SO}(5))=\frac{1}{8} \frac{1}{(2 \pi)^{4}}\left[\left(\operatorname{tr} F_{\mathrm{SO}(5)}^{2}\right)^{2}-2 \operatorname{tr} F_{\mathrm{SO}(5)}^{4}\right] .
$$

In the present situation, $F_{\mathrm{SO}(5)}^{A B}$ contains two pieces,

$$
F_{\mathrm{SO}(5)}^{A B}=F_{\Sigma}^{A B}+F_{\mathrm{ext}}^{A B},
$$

which are given in (5.3), (5.5) respectively. 
We are now in a position to compute $X_{8}$. We only need to collect terms linear in $V_{\Sigma}$. The result reads

$$
\begin{aligned}
X_{8}= & \frac{1}{48}\left(q_{1} \frac{F^{1}}{2 \pi}+q_{2} \frac{F^{2}}{2 \pi}\right)\left[p_{1}\left(T W_{4}\right)+p_{1}\left(\mathrm{SO}(3)_{S^{2}}\right)\right] \frac{V_{\Sigma}}{2 \pi} \\
& -\frac{1}{48}\left[\left(\frac{F^{1}}{2 \pi}\right)^{2}-\left(\frac{F^{2}}{2 \pi}\right)^{2}\right]\left(q_{1} \frac{F^{1}}{2 \pi}-q_{2} \frac{F^{2}}{2 \pi}\right) \frac{V_{\Sigma}}{2 \pi}+\ldots
\end{aligned}
$$

\subsubsection{Inflow anomaly polynomial}

Our first task is the computation of $\int_{M_{6}} E_{4}^{3}$. Notice that in (5.10) the only objects with legs along the $S^{4}$ fibers are $\bar{V}_{4}^{\mathrm{tg}}, \bar{\omega}_{A B}^{\mathrm{tg}}$. The integration of $E_{4}^{3}$ along $S^{4}$ can then be performed using the identities (A.17), (A.18). The integration along the $S^{4}$ fibers yields

$$
\int_{S^{4}} E_{4}^{3}=\frac{1}{4} N^{3} \cdot \frac{1}{8} \frac{1}{(2 \pi)^{4}}\left[\left(\operatorname{tr} \mathcal{F}^{2}\right)^{2}-2 \operatorname{tr} \mathcal{F}^{4}\right]+3 N \gamma_{4}^{2} .
$$

We now have to integrate over the Riemann surface. The term $\gamma_{4}$, however, has no legs along $\Sigma_{g}$, and drops out. The integral over $\Sigma_{g}$ is performed recalling the definition of $\mathcal{F}^{A B}$ in (5.11). The result reads

$$
\int_{M_{6}} E_{4}^{3}=-\frac{1}{8} N^{3}\left(q_{1} \frac{F^{2}}{2 \pi}+q_{2} \frac{F^{1}}{2 \pi}\right)\left[4 \frac{F^{1}}{2 \pi} \frac{F^{2}}{2 \pi}+q_{1} q_{2} \frac{F^{a} F_{a}}{(2 \pi)^{2}}\right] .
$$

The terms with $F^{a}$ are only present is the Riemann surface is a sphere.

Combining the $E_{4}^{3}$ contribution and the $E_{4} X_{8}$ contribution, we get the total inflow anomaly polynomial. In order to facilitate comparison with the CFT expectation, we introduce the notation

$$
\frac{F^{1}}{2 \pi}=-2 n_{1}, \quad \frac{F^{2}}{2 \pi}=-2 n_{2}
$$

We then have

$$
\begin{aligned}
I_{6}^{\text {inflow }}= & -\frac{1}{6} N\left(q_{1} n_{1}^{3}+q_{2} n_{2}^{3}\right)-\frac{2}{3}\left(N^{3}-\frac{1}{4} N\right)\left(q_{1} n_{1} n_{2}^{2}+q_{2} n_{2} n_{1}^{2}\right) \\
& +\frac{1}{24} N\left(q_{1} n_{1}+q_{2} n_{2}\right) p_{1}\left(T W_{4}\right) \\
& -\frac{1}{24}\left[\left(N^{3} q_{2}^{2}-N\right) q_{1} n_{1}+\left(N^{3} q_{1}^{2}-N\right) q_{2} n_{2}\right] p_{1}\left(\mathrm{SO}(3)_{S^{2}}\right) .
\end{aligned}
$$

The first two lines of the previous expression are in accordance with the results quoted in $[10,35]$. The last line is only present when the Riemann surface is a sphere, and at present has not appeared in field-theoretic analyses of this scenario. The decoupling modes that have to be subtracted to obtain the anomaly of the interacting SCFT are given by dimensional reduction on $\Sigma_{g}$ of a free $6 \mathrm{~d}(2,0)$ tensor multiplet, which corresponds to the center of mass mode of the branes. 


\section{2 $\quad S^{2}$ fibrations over a product of Riemann surfaces}

In this subsection we apply the methods of section 3.1 to construct the inflow anomaly polynomial associated to a class of $A d S_{5}$ solutions of 11d supergravity first discussed in [20], which we refer to as GMSW solutions in this work. The input data for the construction of the inflow anomaly polynomial are the geometry of the internal space $M_{6}$ and the closed, gauge-invariant 4-form $V_{4}$ which we use as seed for the construction of $E_{4}$. Both $M_{6}$ and $V_{4}$ are read off from the supergravity solution. The geometry of $M_{6}$ can be directly inferred from the $11 \mathrm{~d}$ line element, while $V_{4}$ is identified, up to normalization, with the $G_{4}$-flux of the solution.

Salient features of the solutions. Let us now discuss some basic properties of $M_{6}$ and $V_{4}$ in the GMSW solutions. We refer the reader to appendix $\mathrm{F}$ for a more detailed review.

The line element of $M_{6}$ is of the form

$$
d s^{2}\left(M_{6}\right)=h_{S^{2}}^{2} d s^{2}\left(S^{2}\right)+h_{\Sigma}^{2} d s^{2}\left(\Sigma_{g}\right)+h_{y}^{2} d y^{2}+h_{\psi}^{2} D \psi^{2} .
$$

Some comments on our notation are in order. The coordinate $y$ parametrizes an interval, $y \in\left[y_{\min }, y_{\max }\right]$, and the metric functions $h_{S^{2}}, h_{\Sigma}, h_{y}, h_{\psi}$ are functions of $y$ only. Their explicit expressions can be extracted from (F.1). The symbol $d s^{2}\left(S^{2}\right)$ denotes the line element on a round $S^{2}$ with unit radius, while $d s^{2}\left(\Sigma_{g}\right)$ is the line element on a Riemann surface of genus $g$ equipped with a constant curvature metric. We only consider the cases $g=0$ or $g \geq 2$, and we normalize the metric in such a way that the Ricci scalar is $R= \pm 2$. The angle $\psi$ has periodicity $2 \pi$. The circle $S_{\psi}^{1}$ is twisted over $S^{2}$ and $\Sigma_{g}$, with ${ }^{7}$

$$
d D \psi=-2 V_{S^{2}}-\chi V_{\Sigma} .
$$

The quantity $\chi$ is the Euler characteristic of $\Sigma_{g}$. The 2-form $V_{S^{2}}$ is proportional to the volume form of $S^{2}$, while $V_{\Sigma}$ is proportional to the volume form on $\Sigma_{g}$. We use the normalization conventions

$$
\int_{S^{2}} V_{S^{2}}=2 \pi, \quad \int_{\Sigma_{g}} V_{\Sigma}=2 \pi
$$

The metric functions $h_{S^{2}}, h_{\Sigma}$ are smooth and strictly positive on the entire $y$ interval. The metric function $h_{y}^{2}$ is everywhere positive on the interior of the $y$ interval, with simple poles at the endpoints. The function $h_{\psi}^{2}$, on the other hand, is everywhere positive on the interior of the $y$ interval, with simple zeros at the endpoints. The $2 \mathrm{~d}$ space obtained combining the $y$ interval with the $\psi$ circle is topologically a 2 -sphere, which we denote $S_{y \psi}^{2}$. The behavior of $h_{y}, h_{\psi}$ at the endpoints of the $y$ interval is such that $S_{y \psi}^{2}$ is free of conical singularities.

The angle $\psi$ is an isometry direction for $M_{6}$. The dual 1 -form reads

$$
k_{\psi}=h_{\psi}^{2} D \psi .
$$

The space $M_{6}$ admits additional $\mathrm{SO}(3)$ isometries originating from $S^{2}$. These isometries are preserved by the $S_{y \psi}^{2}$ fibration on top of $S^{2}$. The corresponding Killing 1-forms are

$$
k_{a}=h_{S^{2}}^{2} \epsilon_{a b c} z^{b} d z^{c}+z_{a} h_{\psi}^{2} D \psi .
$$

\footnotetext{
${ }^{7}$ Compared to [20], we have flipped the sign of $\psi$.
} 
The scalars $z^{a}, a=1,2,3$ are constrained coordinates on $S^{2}$, satisfying $z^{a} z_{a}=1$. If the Riemann surface $\Sigma_{g}$ is also a 2 -sphere, it give rise to a completely analogous set of Killing vectors, generating an extra $\mathrm{SO}(3)$ factor in the isometry group. For simplicity, in the rest of this section we focus on the isometries associated to the angle $\psi$ and the $S^{2}$, and we do not consider the additional isometries that emerge if $\Sigma_{g}$ is also a 2 -sphere.

The form $V_{4}$, which is going to be used as seed in the construction of $E_{4}$ below, is extracted from the expression of the $G_{4}$-flux in the GMSW solution. The form $V_{4}$ can be written as

$$
V_{4}=\left[d \gamma_{\Sigma} \frac{V_{\Sigma}}{2 \pi}+d \gamma_{S^{2}} \frac{V_{S^{2}}}{2 \pi}\right] \frac{D \psi}{2 \pi}-\left[2 \gamma_{\Sigma}+\chi \gamma_{S^{2}}\right] \frac{V_{\Sigma}}{2 \pi} \frac{V_{S^{2}}}{2 \pi} .
$$

In the previous equation, $\gamma_{\Sigma}$ and $\gamma_{S^{2}}$ are functions of $y$ only. The expressions for $\gamma_{\Sigma}, \gamma_{S^{2}}$ can be extracted from in (F.10), (F.5).

Let us stress that the presentation (5.31) of $V_{4}$ in terms of $\gamma_{S^{2}}, \gamma_{\Sigma}$ is subject to a redundancy. More precisely, there is a 1-parameter family of redefinitions of the functions $\gamma_{\Sigma}, \gamma_{S^{2}}$ that leave $V_{4}$ invariant,

$$
\gamma_{S^{2}} \rightarrow \gamma_{S^{2}}+2 K, \quad \gamma_{\Sigma} \rightarrow \gamma_{\Sigma}-\chi K
$$

where $K$ is an arbitrary constant. This redundancy will be fixed below when we construct $E_{4}$ and impose the condition (3.28).

Flux quantization. We can extract the flux quantum numbers of the setup by integrating $V_{4}$ on suitable 4-cycles in $M_{6}$ [26]. If we integrate $V_{4}$ along the Riemann surface $\Sigma_{g}$ and $S_{y \psi}^{2}$, we obtain

$$
N_{\Sigma}:=\int_{\Sigma_{g} \times S_{y \psi}^{2}} V_{4}=\left[\gamma_{\Sigma}\right]_{y=y_{\min }}^{y=y_{\max }}=\gamma_{\Sigma}^{\mathrm{N}}-\gamma_{\Sigma}^{\mathrm{S}} .
$$

The superscripts ' $\mathrm{N}$ ', 'S' denote evaluation at $y=y_{\max }$ min, respectively. We can also integrate $V_{4}$ along $S_{y \psi}^{2}$ and $S^{2}$,

$$
N_{S^{2}}:=\int_{S^{2} \times S_{y \psi}^{2}} V_{4}=\left[\gamma_{S^{2}}\right]_{y=y_{\min }}^{y=y_{\max }}=\gamma_{S^{2}}^{\mathrm{N}}-\gamma_{S^{2}}^{\mathrm{S}} .
$$

Finally, we can integrate $V_{4}$ over $\Sigma_{g} \times S^{2}$ at $y=y_{\max }$ or $y=y_{\min }$,

$$
\begin{aligned}
& N_{\mathrm{N}}:=\int_{y=y_{\max }} V_{4}=-\left[2 \gamma_{\Sigma}^{\mathrm{N}}+\chi \gamma_{S^{2}}^{N}\right], \\
& N_{\mathrm{S}}:=\int_{y=y_{\min }} V_{4}=-\left[2 \gamma_{\Sigma}^{\mathrm{S}}+\chi \gamma_{S^{2}}^{S}\right] .
\end{aligned}
$$

The four quantities $N_{\Sigma}, N_{S^{2}}, N_{\mathrm{N}}, N_{\mathrm{S}}$ are all integers, but they are not independent, since

$$
N_{\mathrm{N}}-N_{\mathrm{S}}+2 N_{\Sigma}+\chi N_{S^{2}}=0 .
$$

Since $\chi$ is an even integer, the difference $N_{\mathrm{N}}-N_{\mathrm{S}}$ is an even integer. It follows that the sum $N_{\mathrm{N}}+N_{\mathrm{S}}$ is also an even integer, and we can thus define the integer $M$ via

$$
M=\frac{1}{2}\left(N_{\mathrm{N}}+N_{\mathrm{S}}\right) .
$$

The integers $\left(N_{S^{2}}, N_{\Sigma}, M\right)$ can be taken to be the independent quanta specifying the $G_{4^{-}}$ flux configuration. 
Harmonic 2-forms on $\boldsymbol{M}_{\mathbf{6}}$. The space $M_{6}$ admits three independent harmonic 2-forms. This is in accordance with the fact that we have three independent flux quanta, associated to the three independent 4-cycles of $M_{6}$. The harmonic 2-forms are denoted $\omega_{\alpha}$ and can be parametrized as

$$
\omega_{\alpha}=d H_{\alpha} \frac{D \psi}{2 \pi}+\left(t_{\alpha S^{2}}-2 H_{\alpha}\right) \frac{V_{S^{2}}}{2 \pi}+\left(t_{\alpha \Sigma}-\chi H_{\alpha}\right) \frac{V_{\Sigma}}{2 \pi},
$$

where $H_{\alpha}$ is a function of $y$ and $t_{\alpha S^{2}}, t_{\alpha \Sigma}$ are suitable constants. This parametrization is subject to a 1-parameter family of redefinitions, corresponding to shifts of $H_{\alpha}$ by an arbitrary constant. For definiteness, we fix this ambiguity by demanding that

$$
H_{\alpha}^{\mathrm{N}}+H_{\alpha}^{\mathrm{S}}=0
$$

The quantities $H_{\alpha}, t_{\alpha S^{2}}, t_{\alpha \Sigma}$ may be fixed in terms of the metric functions in (5.26) by requiring that $\omega_{\alpha}$ be co-closed. This would require solving and ODE for $H_{\alpha}$. To proceed, however, we do not need to find the explicit form of the function $H_{\alpha}$. It is sufficient to demand that the three $\omega_{\alpha}$ 's be Poincaré dual to the three 4-cycles associated to the flux quanta $\left(N_{\Sigma}, N_{S^{2}}, M\right)$. More precisely, we require

$$
\int_{M_{6}} V_{4} \omega_{1}=N_{S^{2}}, \quad \int_{M_{6}} V_{4} \omega_{2}=N_{\Sigma}, \quad \int_{M_{6}} V_{4} \omega_{3}=M .
$$

We compute

$$
\begin{aligned}
\int_{M_{6}} V_{4} \omega_{\alpha} & =\left[t_{\alpha S^{2}} \gamma_{\Sigma}+t_{\alpha \Sigma} \gamma_{S^{2}}-2 \gamma_{\Sigma} H_{\alpha}-\chi \gamma_{S^{2}} H_{\alpha}\right]_{\mathrm{S}}^{\mathrm{N}} \\
& =N_{\Sigma} t_{\alpha S^{2}}+N_{S^{2}} t_{\alpha \Sigma}+2 M H_{\alpha}^{\mathrm{N}}
\end{aligned}
$$

where we have expressed $\gamma_{S^{2}, \Sigma}^{\mathrm{N}, \mathrm{S}}$ in terms of the flux quanta. From (5.41) we see that (5.40) implies

\begin{tabular}{|c|c|c|c|}
\hline & $H_{\alpha}^{\mathrm{N}}$ & $t_{\alpha S^{2}}$ & $t_{\alpha \Sigma}$ \\
\hline$\alpha=1$ & 0 & 0 & 1 \\
$\alpha=2$ & 0 & 1 & 0 \\
$\alpha=3$ & $\frac{1}{2}$ & 0 & 0 \\
\hline
\end{tabular}

The above table contains all information we need about $\omega_{\alpha}$ to compute the inflow anomaly polynomial.

Construction of $\boldsymbol{E}_{4}$. In the construction of $E_{4}$ we introduce background connections for the $\mathrm{U}(1)_{\psi}$ isometry as well as the $\mathrm{SO}(3)$ isometry of $S^{2}$. We also have three background connections $A^{\alpha}$ associated to the three harmonic 2 -forms $\omega_{\alpha}$, even though one combination of these vectors is massive, as discussed in more detail later. The construction of $E_{4}$ proceeds according to the general recipe of section 3.1. The details of the derivation can be found in appendix F. 
The 4 -form $E_{4}$ can be written as

$$
\begin{aligned}
E_{4} & =V_{4}^{\mathrm{eq}}+\frac{F^{\alpha}}{2 \pi} \omega_{\alpha}^{\mathrm{eq}}, \\
V_{4}^{\mathrm{eq}} & =\left(d \gamma_{\Sigma} \frac{V_{\Sigma}}{2 \pi}+d \gamma_{S^{2}} e_{2}^{S^{2}}\right) \frac{(D \psi)^{\mathrm{g}}}{2 \pi}+\left(\gamma_{\Sigma} \frac{V_{\Sigma}}{2 \pi}+\gamma_{S^{2}} e_{2}^{S^{2}}\right)\left(-2 e_{2}^{S^{2}}-\chi \frac{V_{\Sigma}}{2 \pi}+2 \frac{F^{\psi}}{2 \pi}\right), \\
\omega_{\alpha}^{\mathrm{eq}} & =d H_{\alpha} \frac{(D \psi)^{\mathrm{g}}}{2 \pi}+\left(t_{\alpha S^{2}}-2 H_{\alpha}\right) e_{2}^{S^{2}}+\left(t_{\alpha \Sigma}-\chi H_{\alpha}\right) \frac{V_{\Sigma}}{2 \pi}+2 H_{\alpha} \frac{F^{\psi}}{2 \pi} .
\end{aligned}
$$

The 1-form $(D \psi)^{\mathrm{g}}$ is the gauged version of $D \psi$. It is computed in appendix $\mathrm{F}$, and satisfies the property

$$
\frac{d(D \psi)^{\mathrm{g}}}{2 \pi}=-2 e_{2}^{S^{2}}-\chi \frac{V_{\Sigma}}{2 \pi}+2 \frac{F^{\psi}}{2 \pi} .
$$

The quantity $F^{\psi}=d A^{\psi}$ is the external connection associated to the $\mathrm{U}(1)_{\psi}$ isometry. The 2 -form $e_{2}^{S^{2}}$ is the closed and $\mathrm{SO}(3)$-invariant completion of $V_{S^{2}} /(2 \pi)$,

$$
d e_{2}^{S^{2}}=0, \quad \int_{S^{2}} e_{2}^{S^{2}}=1
$$

The explicit expression of $e_{2}^{S^{2}}$ can be found in appendix F.

Recall from section 3.2 that we must impose the relation (3.28) in order to be able to set to zero the combination $N_{\alpha} F^{\alpha}$ of background field strengths associated to harmonic 2 -forms. As detailed in appendix F, imposing (3.28) allows us to write down the values of the functions $\gamma_{S^{2}}, \gamma_{\Sigma}$ at the endpoints of the $y$ interval in terms of the three flux quanta $N_{S^{2}}, N_{\Sigma}, M$,

$$
\gamma_{S^{2}}^{\mathrm{N}, \mathrm{S}}=\frac{M N_{S^{2}}}{2 N_{\Sigma}-\chi N_{S^{2}}} \pm \frac{1}{2} N_{S^{2}}, \quad \gamma_{\Sigma}^{\mathrm{N}, \mathrm{S}}=-\frac{M N_{\Sigma}}{2 N_{\Sigma}-\chi N_{S^{2}}} \pm \frac{1}{2} N_{\Sigma}
$$

Computation of $\boldsymbol{X}_{\mathbf{8}}$. The first Pontryagin class $p_{1}\left(T M_{11}\right)$ takes the form

$$
p_{1}\left(T M_{11}\right)=p_{1}\left(T W_{4}\right)+p_{1}(\mathrm{SO}(3))+\left[-2 e_{2}^{S^{2}}-\chi \frac{V_{\Sigma}}{2 \pi}+2 \frac{F^{\psi}}{2 \pi}\right]^{2} .
$$

The above relation is justified as follows. The internal space $M_{6}$ is an $S_{\psi}^{1}$ fibration over a $5 \mathrm{~d}$ space. Moreover, $M_{6}$ is in turn fibered over external spacetime $W_{4}$. The terms $p_{1}\left(T W_{4}\right)+p_{1}(\mathrm{SO}(3))$ capture the first Pontryagin class of the $5 \mathrm{~d}$ space fibered over $W_{4}$. The class $p_{1}(\mathrm{SO}(3))$ is associated to the $\mathrm{SO}(3)$ isometry of $S^{2}$. The final contribution is equal to $\left[d(D \psi)^{\mathrm{g}} /(2 \pi)\right]^{2}$. It accounts for the Chern root associated to the $S_{\psi}^{1}$ fibration, whose connection has both internal legs (on $S^{2}$ and $\Sigma_{g}$ ) as well as external legs on $W_{4}$. By a similar token, the second Pontryagin class of the total geometry reads

$$
p_{2}\left(T M_{11}\right)=\left[p_{1}\left(T W_{4}\right)+p_{1}(\mathrm{SO}(3))\right]\left[-2 e_{2}^{S^{2}}-\chi \frac{V_{\Sigma}}{2 \pi}+2 \frac{F^{\psi}}{2 \pi}\right]^{2} .
$$

Notice that we can drop any term in $p_{2}\left(T M_{11}\right)$ with more than six external legs. In summary, the class $X_{8}$ for the setup under examination takes the form

$$
X_{8}=\frac{1}{192}\left\{p_{1}\left(T W_{4}\right)+p_{1}(\mathrm{SO}(3))-\left[-2 e_{2}^{S^{2}}-\chi \frac{V_{\Sigma}}{2 \pi}+2 \frac{F^{\psi}}{2 \pi}\right]^{2}\right\}^{2} .
$$


Inflow anomaly polynomial. We can now compute $\int_{M_{6}} E_{4}^{3}$ and $\int_{M_{6}} E_{4} X_{8}$ and extract the inflow anomaly polynomial. Integrals over $S^{2}$ are conveniently performed with the help of the Bott-Cattaneo formula, reviewed in appendix F. We also need (5.42) and (5.46).

The curvatures associated to the three harmonic 2 -forms $\omega_{\alpha}$ are subject to the constraint

$$
N_{\alpha} F^{\alpha}=N_{S^{2}} F^{1}+N_{\Sigma} F^{2}+M F^{3}=0 .
$$

We choose to give the result in terms of $F^{2}$ and $F^{3}$, solving the above constraint for $F^{1}$.

The inflow anomaly polynomial reads

$$
\begin{aligned}
(2 \pi)^{3} I_{6}^{\text {inflow }}= & -\frac{1}{24}\left(\chi N_{S^{2}}+2 N_{\Sigma}\right) p_{1}\left(T W_{4}\right) F^{\psi}+\frac{1}{24} \chi p_{1}\left(T W_{4}\right) F^{3} \\
& +\left[\frac{N_{S^{2}}^{2}\left(12 M^{2}+4 \chi N_{S^{2}} N_{\Sigma}+\chi^{2} N_{S^{2}}^{2}-12 N_{\Sigma}^{2}\right)}{24\left(\chi N_{S^{2}}-2 N_{\Sigma}\right)}+\frac{1}{12} \chi N_{S^{2}}\right] p_{1}(\mathrm{SO}(3)) F^{\psi} \\
& -\frac{1}{4} M N_{S^{2}} p_{1}(\mathrm{SO}(3)) F^{2}-\frac{1}{8} N_{S^{2}}\left(\chi N_{S^{2}}+2 N_{\Sigma}\right) p_{1}(\mathrm{SO}(3)) F^{3} \\
& +\frac{1}{6}\left(\chi N_{S^{2}}+2 N_{\Sigma}\right)\left(F^{\psi}\right)^{3} \\
& +\left[-\frac{N_{S^{2}} N_{\Sigma}\left(-2 M-\chi N_{S^{2}}+2 N_{\Sigma}\right)\left(2 M-\chi N_{S^{2}}+2 N_{\Sigma}\right)}{\left(2 N_{\Sigma}-\chi N_{S^{2}}\right)^{2}}-\frac{1}{2} \chi\right]\left(F^{\psi}\right)^{2} F^{3} \\
& +\frac{4 M N_{\Sigma}}{2 N_{\Sigma}-\chi N_{S^{2}}} F^{\psi} F^{2} F^{3}+\frac{4 M^{2}-\chi^{2} N_{S^{2}}^{2}+4 N_{\Sigma}^{2}}{2\left(2 N_{\Sigma}-\chi N_{S^{2}}\right)} F^{\psi}\left(F^{3}\right)^{2} \\
& +\frac{N_{\Sigma}}{N_{S^{2}}}\left(F^{2}\right)^{2} F^{3}+\frac{M}{N_{S^{2}}} F^{2}\left(F^{3}\right)^{2}-\frac{1}{6} \chi\left(F^{3}\right)^{3} .
\end{aligned}
$$

An alternative presentation is based on a different choice of basis of harmonic 2-forms, which we denote $\omega_{\mathrm{C}}, \omega_{\mathrm{N}}, \omega_{\mathrm{S}}$. These combinations of the three $\omega_{\alpha}$ 's are defined by

$$
\int V_{4} \omega_{\mathrm{C}}=N_{S^{2}}, \quad \int V_{4} \omega_{\mathrm{N}, \mathrm{S}}=N_{\mathrm{N}, \mathrm{S}},
$$

where $N_{\mathrm{N}, \mathrm{S}}$ were defined in (5.34). More explicitly,

$$
\omega_{\mathrm{C}}=\omega_{1}, \quad \omega_{\mathrm{N}, \mathrm{S}}=\mp \frac{\chi}{2} \omega_{1} \mp \omega_{2}+\omega_{3} .
$$

Correspondingly, we have the identifications

$$
F^{2}=-F^{\mathrm{N}}+F^{\mathrm{S}}, \quad F^{3}=F^{\mathrm{N}}+F^{\mathrm{S}} .
$$

If desired, it is straightforward to rewrite the anomaly polynomial (5.51) in terms of $F^{\mathrm{N}, \mathrm{S}}$.

Exact superconformal R-symmetry and central charge at large $\boldsymbol{N}$. To identify the superconformal R-symmetry we use $a$-maximization [36]. The non-Abelian flavor symmetry $\mathrm{SO}(3)$ cannot participate to $a$-maximization. As a result, we simply turn off the associated background curvature. At the level of the anomaly polynomial, we perform the replacements

$$
F^{\psi} \rightarrow F^{\mathrm{R}}, \quad F^{2} \rightarrow s^{2} F^{\mathrm{R}}, \quad F^{3} \rightarrow s^{3} F^{\mathrm{R}},
$$


with unspecified coefficients $s^{2,3}$. For simplicity, we work in the large $N$ limit, with the scalings

$$
N_{S^{2}} \sim N_{\Sigma} \sim M \sim F^{2} \sim F^{3} \sim \mathcal{O}(N) .
$$

In the large $N$ approximation, our task is to maximize the coefficient of $\left(F^{\mathrm{R}}\right)^{3}$. There are four branches of solutions for $s^{2,3}$. In two branches, the coefficient of $\left(F^{\mathrm{R}}\right)^{3}$ attains the value 0 ; these branches are not acceptable. On the other two branches, we find

$$
(2 \pi)^{3} I_{6}^{\mathrm{CFT}}=(2 \pi)^{3}\left(-I_{6}^{\text {inflow }}\right)=\frac{1}{6} \operatorname{tr} \mathrm{R}^{3}\left(F^{\mathrm{R}}\right)^{3},
$$

with

$$
\begin{aligned}
\operatorname{tr} \mathrm{R}^{3}= & \pm \frac{8 N_{S^{2}}^{2} N_{\Sigma}^{2}\left(4 N_{\Sigma}^{2}+2 \chi N_{S^{2}} N_{\Sigma}+\chi^{2} N_{S^{2}}^{2}-3 M^{2}\right)^{3 / 2}}{\left(2 \chi N_{S^{2}} N_{\Sigma}+3 M^{2}\right)^{2}} \\
& -\frac{4 N_{S^{2}}^{2} N_{\Sigma}^{2}\left(2 N_{\Sigma}+\chi N_{S^{2}}\right)\left(8 N_{\Sigma}^{2}+2 \chi N_{S^{2}} N_{\Sigma}+2 \chi^{2} N_{S^{2}}^{2}-9 M^{2}\right)}{\left(2 \chi N_{S^{2}} N_{\Sigma}+3 M^{2}\right)^{2}}
\end{aligned}
$$

At large $N$,

$$
a=c=\frac{9}{32} \operatorname{tr} \mathrm{R}^{3} .
$$

If we select the branch with the plus sign, we find

$$
\begin{aligned}
c= & \frac{9 N_{S^{2}}^{2} N_{\Sigma}^{2}\left(4 N_{\Sigma}^{2}+2 \chi N_{S^{2}} N_{\Sigma}+\chi^{2} N_{S^{2}}^{2}-3 M^{2}\right)^{3 / 2}}{4\left(2 \chi N_{S^{2}} N_{\Sigma}+3 M^{2}\right)^{2}} \\
& -\frac{9 N_{S^{2}}^{2} N_{\Sigma}^{2}\left(2 N_{\Sigma}+\chi N_{S^{2}}\right)\left(8 N_{\Sigma}^{2}+2 \chi N_{S^{2}} N_{\Sigma}+2 \chi^{2} N_{S^{2}}^{2}-9 M^{2}\right)}{8\left(2 \chi N_{S^{2}} N_{\Sigma}+3 M^{2}\right)^{2}} .
\end{aligned}
$$

We verify in appendix $\mathrm{F}$ that this result agrees with the holographic central charge computed in [26]. More precisely, the explicit formula given in [26] applies to solutions with $M=0$. The formula (5.60) can be regarded as the generalization to the case $M \neq 0$, which is harder to tackle directly in holography.

Here we focused on a large- $N$ test of our result. Nonetheless, we expect the inflow anomaly polynomial (5.51) to be exact in $N$, but to also contain contributions from decoupled modes. A field-theoretic understanding of the latter would allow us to repeat the $a$-maximization analysis to obtain corrections to the central charge of the CFT (5.60).

\section{Discussion}

We have presented a systematic method of computing anomalies of QFTs that are geometrically engineered in M-theory, using anomaly inflow in the M-theory background. As we have described, there are two main pieces of data which determine the inflow analysis (i.e., ingredients of $\mathcal{I}_{12}$ ): the value of $G_{4}$ on the boundary of $11 \mathrm{~d}$ spacetime, which we denote by $E_{4}$, and the topology of the space $M_{10-d}$ corresponding to the transverse directions to the $d$-dimensional QFT worldvolume. We presented a general recipe for constructing $E_{4}$ in terms of forms in $M_{10-d}$, and characterized its ambiguities. This is naturally done using the language of $G$-equivariant cohomology, where $G$ is the isometry group of $M_{10-d}$. 
We have argued that the inflow anomaly polynomial can be extracted unambiguously in $d=2,4,6$. For the remainder of this discussion, we elaborate on some of the results we have obtained by applying this formalism.

All the ambiguity in our construction of $E_{4}$ can be encapsulated by a single external 4 -form $\gamma_{4}$. We argue in appendix $\mathrm{C}$ that inflow anomalies in two and four dimensions are independent of $\gamma_{4}$. For $d=6$, the inflow anomaly polynomial $I_{8}^{\text {inflow }}$ depends on $\gamma_{4}$, which is fixed by extremizing $I_{8}^{\text {inflow }}$. This prescription is equivalent to imposing that the 8-form $E_{4}^{2}+2 X_{8}$ be trivial in the cohomology of the internal space $M_{4}$. We interpret this requirement on $E_{4}^{2}+2 X_{8}$ as a consequence of tadpole cancellation, which is necessary to have a well-defined M-theory setup. Let us stress that our prescription for fixing $\gamma_{4}$ in $d=6$ is such that we obtain the correct answer for the anomaly polynomial for M5-branes probing $\mathbb{C}^{2} / \Gamma$ (given in (4.16)). In particular, the inclusion of $\gamma_{4}$ generates an additional term relative to the inflow analysis of [24], which provides precisely the contribution of the Green-Schwarz term of the center of mass mode for the M5-branes. Previously this term had only been fixed via anomaly matching on the tensor branch.

Turning to the 4d SCFTs corresponding to BBBW solutions, we have noted that our inflow analysis yields a new set of terms in the anomaly polynomials for the case of M5-branes compactified on a sphere (the last line of (5.25)). These terms are due to the additional $\mathfrak{s u}(2)$ isometry algebra of the sphere. This has not previously been discussed in the literature - such a symmetry does not appear from reducing the anomaly polynomial from $6 \mathrm{~d}[9$, 10], and is missing in the analysis of the flow to these theories from closing punctures on the surface [37-39]. From the latter point of view, this $\mathfrak{s u}(2)$ is an accidental symmetry in the IR. It would be interesting to understand if some of the subtleties regarding decoupled operators in these theories are clarified with the knowledge of this IR symmetry enhancement.

We have also outlined a connection between the data of the holographic supergravity solutions and the input to the inflow anomaly polynomial, and we demonstrated the utility of this observation by computing the anomaly polynomial of the $4 \mathrm{~d}$ field theories dual to the GMSW solutions (with result given in (5.51)). Let us contrast our method to the standard application of the AdS/CFT dictionary. In the latter, anomalies are extracted by computing Chern-Simons coefficients in the bulk; subleading terms in $N$ require computing higher derivative corrections to the supergravity action and loops in AdS. In our approach, the inflow anomaly polynomial is expected to be exact in $N$, but it contains contributions both from the interacting CFT of interest and from decoupled sectors. Our analysis of GMSW solutions gives us strong hints for a UV realization of the dual SCFTs in terms of M5-branes probing a $\mathbb{C}^{2} / \mathbb{Z}_{2}$ singularity, compactified on a Riemann surface with a suitable flavor twist [40]. The field theory picture will shed light on decoupling modes for these setups.

There are many interesting future directions to explore. We expect our methods to be applicable to a wider class of $6 \mathrm{~d}$ theories constructed in M-theory, including $(2,0)$ theories of type $D_{N}$ and $(1,0)$ E-string theories. We also believe that our approach can be extended to setups with M5-branes wrapped on a Riemann surface with defects. This analysis has been performed in $[41,42]$ for regular punctures in $4 \mathrm{~d} \mathcal{N}=2$ theories, and it would be interesting to study $\mathcal{N}=2$ irregular punctures and $\mathcal{N}=1$ punctures for general compactifications of $6 \mathrm{~d}$ theories on a Riemann surface. From a broader perspective, it would be useful to 
develop systematic geometric tools for the computation of 't Hooft anomalies of theories engineered in type IIA, type IIB string theories and F-theory. Moreover, the methods of this work can be straightforwardly generalized to include anomalies for continuous higherform symmetries [43, 44].

\section{Acknowledgments}

We would like to thank Fabio Apruzzi, Nikolay Bobev, Simone Giacomelli, Ken Intriligator, Craig Lawrie, Raffaele Savelli, Sakura Schäfer-Nameki, Alessandro Tomasiello for interesting conversations and correspondence. The work of IB and FB is supported in part by NSF grant PHY-1820784. RM is supported in part by ERC Grant 787320 - QBH Structure. We gratefully acknowledge the Aspen Center for Physics, supported by NSF grant PHY-1607611, for hospitality during part of this work.

\section{A Some useful identities}

\section{A.1 Identities for gauging of isometries}

Let $k_{I}^{m}$ denote the Killing vectors of the internal space $M_{10-d}$, satisfying $£_{I} k_{J}=f_{I J}{ }^{K} k_{K}$. Given a $p$-form $\omega$ on $M_{10-d}$, its gauged counterpart is denoted $\omega^{\mathrm{g}}$ and is defined by

$$
\omega=\frac{1}{p !} \omega_{m_{1} \ldots m_{p}} d \xi^{m_{1}} \ldots d \xi^{m_{p}} \quad \Rightarrow \quad \omega^{\mathrm{g}}=\frac{1}{p !} \omega_{m_{1} \ldots m_{p}} D \xi^{m_{1}} \ldots D \xi^{m_{p}}
$$

where $D \xi^{m}=d \xi^{m}+k_{I}^{m} A^{I}$. An alternative equivalent presentation of $\omega^{\mathrm{g}}$ is

$$
\omega^{\mathrm{g}}=\sum_{M=0}^{p} \frac{1}{M !} A^{I_{1}} \ldots A^{I_{M}} \iota_{I_{M}} \ldots \iota_{I_{1}} \omega
$$

where $\iota_{I}$ denotes interior product with the Killing vector $k_{I}^{m}$,

$$
\iota_{I} \omega=\frac{1}{(p-1) !} k_{I}^{n} \omega_{n m_{1} \ldots m_{p-1}} d \xi^{m_{1}} \ldots d \xi^{m_{p-1}} .
$$

A natural notion of gauge transformation on $\omega^{\mathrm{g}}$ can be defined as follows. Let $\lambda^{I}$ be a set of scalar functions depending on the external coordinates only, and consider the vector field $\Xi(\lambda)$ in the total space $M_{10}$ specified by $\Xi(\lambda)=\lambda^{I} k_{I}^{m} \partial_{\xi^{m}}$. We may then define ${ }^{8}$

$$
\delta_{\lambda}\left(\omega^{\mathrm{g}}\right)=£_{\Xi(\lambda)}\left(\omega^{\mathrm{g}}\right)+\delta_{\lambda} A^{I} \frac{\delta}{\delta A^{I}}\left(\omega^{\mathrm{g}}\right),
$$

where $\delta_{\lambda} A^{I}$ denotes the standard gauge transformation of a connection,

$$
\delta_{\lambda} A^{I}=-D \lambda^{I}, \quad D \lambda^{I}=d \lambda^{I}-f_{J K}{ }^{I} A^{J} \lambda^{K} .
$$

${ }^{8}$ With reference to (A.2), we have explicitly

$$
\delta_{\lambda} A^{I} \frac{\delta}{\delta A^{I}}\left(\omega^{\mathrm{g}}\right)=\sum_{M=1}^{p} \frac{1}{(M-1) !} \delta_{\lambda} A^{I_{1}} A^{I_{2}} \ldots A^{I_{M}} \iota_{I_{M}} \ldots \iota_{I_{1}} \omega .
$$


Making use of the identity

$$
£_{I} \iota_{J}-\iota_{J} £_{I}=f_{I J}{ }^{K} \iota_{K},
$$

we verify the relation

$$
\delta_{\lambda}\left(\omega^{\mathrm{g}}\right)=\lambda^{I}\left(£_{I} \omega\right)^{\mathrm{g}} .
$$

Let us now suppose that the form $\omega$ is invariant under Lie derivative with respect to all isometry directions,

$$
£_{I} \omega=0 .
$$

Under this assumption, the following identity holds,

$$
d\left(\omega^{\mathrm{g}}\right)=(d \omega)^{\mathrm{g}}+F^{I}\left(\iota_{I} \omega\right)^{\mathrm{g}},
$$

where

$$
F^{I}=d A^{I}-\frac{1}{2} f_{J K}{ }^{I} A^{J} A^{K} .
$$

We may now consider a collection of $p$-forms $\omega_{I}$ that satisfies

$$
£_{I} \omega_{J}=f_{I J}{ }^{K} \omega_{K} .
$$

In other words, $\omega_{I}$ transform in the adjoint representation of the isometry algebra. For such a collection of $p$-forms, one has

$$
d\left(\omega_{I}^{\mathrm{g}}\right)=\left(d \omega_{I}\right)^{\mathrm{g}}+F^{J}\left(\iota_{J} \omega_{I}\right)^{\mathrm{g}}+f_{I J}{ }^{K} A^{J} \omega_{K}^{\mathrm{g}} .
$$

A similar formula holds for a two-indexed collection of $p$-forms: under the assumption that

$$
£_{I} \omega_{J_{1} J_{2}}=f_{I J_{1}}{ }^{K} \omega_{K J_{2}}+f_{I J_{2}}{ }^{K} \omega_{K_{1} K},
$$

one has the identity

$$
d\left(\omega_{I_{1} I_{2}}^{\mathrm{g}}\right)=\left(d \omega_{I_{1} I_{2}}\right)^{\mathrm{g}}+F^{J}\left(\iota_{J} \omega_{I_{1} I_{2}}\right)^{\mathrm{g}}+f_{I_{1} J}{ }^{K} A^{J} \omega_{K I_{2}}^{\mathrm{g}}+f_{I_{2} J} K A^{J} \omega_{I_{1} K}^{\mathrm{g}} .
$$

The relations (A.9), (A.12), (A.14) are all examples of the general identity

$$
d\left(\Lambda_{\mathrm{g}}\right)+A^{I}\left(£_{I} \Lambda^{\mathrm{g}}\right)=(d \Lambda)^{\mathrm{g}}+F^{I}\left(\iota_{I} \Lambda\right)^{\mathrm{g}},
$$

where $\Lambda$ is a $p$-form on $M_{10-d}$ in an arbitrary representation of the isometry algebra.

\section{A.2 Identities for $\mathrm{SO}(5)$ isometry of $S^{4}$}

The forms $\bar{V}_{4}, \bar{\omega}_{A B}, \bar{\sigma}_{A B, C D}$ are defined in (4.5), repeated here for convenience,

$$
\begin{aligned}
\bar{V}_{4} & =\frac{3 N}{8 \pi^{2}} \cdot \frac{1}{4 !} \epsilon_{A_{1} \ldots A_{5}} d y^{A_{1}} d y^{A_{2}} d y^{A_{3}} d y^{A_{4}} y^{A_{5}}, \\
\bar{\omega}_{A B} & =\frac{3 N}{8 \pi^{2}} \cdot \frac{-2}{4 !} \epsilon_{A B C_{1} C_{2} C_{3}} d y^{C_{1}} d y^{C_{2}} y^{C_{3}}, \\
\bar{\sigma}_{A B, C D} & =\frac{3 N}{8 \pi^{2}} \cdot \frac{1}{4 !} \epsilon_{A B C D E} y^{E} .
\end{aligned}
$$


Some useful integral identities involving $\bar{V}_{4}, \bar{\omega}_{A B}, \bar{\sigma}_{A B, C D}$ are

$$
\int_{S_{4}} \bar{\omega}_{A B} \bar{\omega}_{C D}=0, \quad \int_{S^{4}} \bar{V}_{4} \bar{\sigma}_{A B, C D}=0,
$$

as well as

$$
\begin{gathered}
\alpha^{A_{1} A_{2} A_{3} A_{4}} \beta^{B_{1} B_{2} B_{3} B_{4}} \int_{S^{4}} \bar{V}_{4} \bar{\sigma}_{A_{1} A_{2}, A_{3} A_{4}} \bar{\sigma}_{B_{1} B_{2} B_{3} B_{4}}= \\
=N^{3}\left[\frac{3}{8 \pi^{2}}\right]^{2}\left\{\frac{1}{360} \alpha^{A B C D} \beta_{A B C D}-\frac{1}{180} \alpha^{A B C D} \beta_{A C B D}\right\} \\
\alpha^{A_{1} A_{2}} \beta^{B_{1} B_{2}} \gamma^{C_{1} C_{2} C_{3} C_{4}} \int_{S^{4}} \bar{\omega}_{A_{1} A_{2}} \bar{\omega}_{B_{1} B_{2}} \bar{\sigma}_{C_{1} C_{2}, C_{3} C_{4}} \\
=N^{3}\left[\frac{3}{8 \pi^{2}}\right]^{2}\left\{\frac{1}{540} \alpha^{A B} \beta^{C D} \gamma_{A B C D}-\frac{1}{270} \alpha^{A B} \beta^{C D} \gamma_{A C B D}\right\} .
\end{gathered}
$$

In the last expressions, the quantities $\alpha, \beta, \gamma$ are arbitrary tensors used as placeholders for $\mathrm{SO}(5)$ indices.

In the main text we described $S^{4}$ in terms of embedding coordinates $y^{A}, A=1, \ldots, 5$. We can also describe $S^{4}$ in terms of four local coordinates $\xi^{m}, m=1, \ldots, 4$. We can write

$$
d y^{A}=\partial_{m} y^{A} d \xi^{m}, \quad\left(d y^{A}\right)^{\mathrm{g}}=\partial_{m} y^{A} D \xi^{m}, \quad D \xi^{m}=d \xi^{m}+k_{A B}^{m} A^{A B},
$$

where $k_{A B}^{m}$ are the Killing vectors of the $\mathrm{SO}(5)$ isometries. They are given by

$$
k^{m A B}=g^{m n} y^{[A} \partial_{n} y^{B]},
$$

where $g_{m n}$ is the round metric on $S^{4}$ induced from the flat metric on $\mathbb{R}^{5}$,

$$
g_{m n}=\partial_{m} y^{A} \partial_{n} y_{A} .
$$

Let us record the useful identities

$$
g^{m n} \partial_{m} y^{A} \partial_{n} y^{B}=\delta^{A B}-y^{A} y^{B}, \quad \iota_{A B} d y^{C}=y_{[A} \delta_{B]}^{C} .
$$

\section{B The $G$-equivariant cohomology class defined by $E_{4}$}

\section{B.1 Relation between $V_{4}^{\mathrm{eq}}, \omega_{\alpha}^{\mathrm{eq}}$ and $G$-equivariant polyforms}

The discussion of section 3.1 fits naturally into the language of $G$-equivariant cohomology, see e.g. [45] for a review. The group $G$ in our discussion is the isometry group of $M_{10-d}$, acting on $M_{10-d}$ infinitesimally via Lie derivative. The objects of interest are maps from the Lie algebra $\mathfrak{g}$ of $G$ into polyforms on $M_{10-d}$, i.e. formal linear combinations of differential forms of various degrees,

$$
\begin{aligned}
f: \mathfrak{g} & \rightarrow \Omega^{*}\left(M_{10-d}\right) \\
\mathcal{X} & \mapsto \alpha(\mathcal{X}) .
\end{aligned}
$$

We fix a basis $\left\{t_{I}\right\}$ of $\mathfrak{g}$, so that we can write

$$
\mathfrak{g} \ni \mathcal{X}=\mathcal{X}^{I} t_{I}
$$


The map $f$ must be $G$-equivariant, which, at the infinitesimal level, amounts to the property

$$
£_{I} f(\mathcal{X})=f_{I J}{ }^{K} \mathcal{X}^{J} \frac{\partial}{\partial \mathcal{X}^{K}} f(\mathcal{X}) .
$$

The equivariant differential acting on $\alpha$ is defined by

$$
\left(d_{\text {eq }} f\right)(\mathcal{X})=d(f(\mathcal{X}))+\iota \mathcal{X} f(\mathcal{X})
$$

where the operation $\iota \mathcal{X}$ amounts to $\mathcal{X}^{I} \iota_{I}$. Crucially, $\left(d_{\mathrm{eq}}\right)^{2}=0$. The $G$-equivariant cohomology of $M_{10-d}$ is then realized by considering the set of $d_{\mathrm{eq}}$-closed polyforms, modulo $d_{\text {eq-exact polyforms. }}$

Let us now revisit the expression (3.7) for the object $V_{4}^{\text {eq }}$. If we identify the external connections $F^{I}$ with the abstract variables $\mathcal{X}^{I}$ parametrizing $\mathfrak{g}$, we can reinterpret $V_{4}^{\text {eq }}$ as a map of the form (B.1),

$$
f_{V_{4}}: \mathcal{X}^{I} \mapsto f_{V_{4}}(\mathcal{X})=V_{4}+\mathcal{X}^{I} \omega_{I}+\mathcal{X}^{I} \mathcal{X}^{J} \sigma_{I J}
$$

We then verify that the conditions (3.10) are precisely equivalent to the equivariance of $f_{V_{4}}$ as in (B.3). Furthermore, the conditions (3.12) are equivalent to $d_{\text {eq }} f_{V_{4}}=0$. The object $V_{4}^{\text {eq }}$ thus amounts to an equivariantly closed form, hence the label 'eq'. In a completely analogous fashion, the object $\omega_{\alpha}^{\mathrm{eq}}$ in (3.13) corresponds to the map

$$
f_{\omega_{\alpha}}: \mathcal{X}^{I} \mapsto f_{\omega_{\alpha}}(\mathcal{X})=\omega_{\alpha}+\mathcal{X}^{I} 2 \sigma_{I \alpha} .
$$

We verify that (3.14) is equivalent to the equivariance of $f_{\omega_{\alpha}}$, and that (3.15) is equivalent to $d_{\mathrm{eq}} f_{\omega_{\alpha}}=0$.

Incidentally, we notice that both $f_{V_{4}}$ and $f_{\omega_{\alpha}}$ are polynomials in $\mathcal{X}^{I}$. The natural notion of degree for each monomial in $f_{V_{4}}$ or $f_{\omega_{\alpha}}$ is

$$
\text { (differential form degree) }+2 \text { (polynomial degree) } \text {. }
$$

It follows that $f_{V_{4}}$ is homogeneous of degree 4 , and $f_{\omega_{\alpha}}$ is homogeneous of degree 2.

\section{B.2 Deformations of $E_{4}$ and $G$-equivariant cohomology}

In section 3.1 we have demonstrated that constructing a good representative for $E_{4}$ amounts to solving the conditions (3.19) and (3.20), repeated here for convenience,

$$
\begin{aligned}
d V_{4} & =0, & £_{X} V_{4} & =0, \\
\iota_{X} V_{4}+d \omega_{X} & =0, & £_{X} \omega_{Y} & =f_{X Y}{ }^{Z} \omega_{Z}, \\
\left.\iota_{(X} \omega_{Y}\right)+d \sigma_{X Y} & =0, & £_{X} \sigma_{Y_{1} Y_{2}} & =f_{X Y_{1}}{ }^{Z} \sigma_{Z Y_{2}}+f_{X Y_{2}}{ }^{Z} \sigma_{Y_{1} Z} .
\end{aligned}
$$

We are using a collective index $X=(I, \alpha)$ that enumerates all external connections. By definition, $\iota_{\alpha}=£_{\alpha}=0$, and the only non-zero components of $f_{X Y} Z$ are $f_{I J}{ }^{K}$. In this appendix, we suppose to fix a reference solution $\left(V_{4}, \omega_{X}, \sigma_{X Y}\right)$ to (B.8), and we investigate the most general deformation to a different solution. 
The most general deformation. The outcome of the analysis is as follows. The new forms are given by

$$
\begin{aligned}
V_{4} & \rightarrow V_{4}+d W_{3} \\
\omega_{X} & \rightarrow \omega_{X}+\iota_{X} W_{3}-Z_{2 X}+d \lambda_{X}+H_{2 X}, \\
\sigma_{X Y} & \rightarrow \sigma_{X Y}+\iota_{(X} \lambda_{Y)}-Z_{0(X Y)}^{\prime}-Z_{0(X Y)}+u_{X Y} .
\end{aligned}
$$

The 3-form $W_{3}$ must be chosen in such a way that there exists a 2 -form $Z_{2 X}$ such that

$$
£_{X} W_{3}=d Z_{2 X}
$$

The 2-form $Z_{2 X}$ in turn determines the 1-forms $Z_{1[X Y]}$ and the harmonic 2-forms $H_{2[X Y]}^{\prime}$ via

$$
f_{X Y} Z_{2 Z}-£_{X} Z_{2 Y}+£_{Y} Z_{2 X}=d Z_{1 X Y}+H_{2 X Y}^{\prime}
$$

The harmonic forms $H_{2 X}$ must be chosen compatibly with the constraint

$$
f_{X Y}{ }^{Z} H_{2 Z}+H_{2 X Y}^{\prime}=0 .
$$

Once the harmonic 2-forms $H_{2 X}$ are chosen, they determine the 0 -forms $Z_{0 X Y}^{\prime}$ and the harmonic 1-forms $H_{1 X Y}^{\prime}$ via

$$
\iota_{X} H_{2 Y}=d Z_{0 X Y}^{\prime}+H_{1 X Y}^{\prime}
$$

The 1-forms $\lambda_{X}$ must be chosen in such a way that there exist a 0 -form $Z_{0 X Y}$ such that

$$
£_{X} \lambda_{Y}=f_{X Y}{ }^{Z} \lambda_{Z}+\iota_{Y} Z_{2 X}-Z_{1 X Y}+d Z_{0 X Y}-H_{1 X Y}^{\prime} .
$$

Finally, the constants $u_{X Y}$ must be chosen in such a way that

$$
\begin{aligned}
0= & £_{\left(Y_{1}\right.} Z_{\left.0 X \mid Y_{2}\right)}-£_{X} Z_{0\left(Y_{1} Y_{2}\right)}+f_{X Y_{1}}{ }^{Z} Z_{0\left(Z Y_{2}\right)}+f_{X Y_{2}}{ }^{Z} Z_{0\left(Z Y_{1}\right)} \\
& -£_{X} Z_{0\left(Y_{1} Y_{2}\right)}^{\prime}+f_{X Y_{1}}{ }^{Z} Z_{0\left(Z Y_{2}\right)}^{\prime}+f_{X Y_{2}}{ }^{Z} Z_{0\left(Z Y_{1}\right)}^{\prime} \\
& -\iota_{\left(Y_{1}\right.} H_{\left.1 X \mid Y_{2}\right)}^{\prime}-\iota_{\left(Y_{1}\right.} Z_{\left.1 X \mid Y_{2}\right)}-f_{X Y_{1}}{ }^{Z} u_{Z Y_{2}}-f_{X Y_{2}}{ }^{Z} u_{Z Y_{1}} .
\end{aligned}
$$

Manifestly symmetric deformations and $G$-equivariant cohomology. It is natural to consider deformations that are parametrized by quantities that are manifestly symmetric under the action of the isometry group $G$. More explicitly, we impose

$$
\begin{aligned}
£_{X} W_{3}=0, \quad £_{X} \lambda_{Y} & =f_{X Y}{ }^{Z} \lambda_{Z}, \quad £_{X} H_{2 Y}=f_{X Y}{ }^{Z} H_{2 Z}, \\
£_{X} u_{Y_{1} Y_{2}} & =f_{X Y_{1}}{ }^{Z} u_{Z Y_{2}}+f_{X Y_{2}}{ }^{Z} u_{Y_{1} Z} .
\end{aligned}
$$

We notice that, in the above equations, we actually have $£_{X} H_{2 Y}=0$ (because $H_{2 Y}$ is harmonic) and $£_{X} u_{Y_{1} Y_{2}}=0$ (because $u_{Y_{1} Y_{2}}$ is constant). Under the additional assumptions (B.16), the most general deformation described above takes a simpler form. Comparison of (B.16) with (B.10) shows that we can take $Z_{2 X}=0$, and therefore also $Z_{1 X Y}=0$, $H_{2 X Y}^{\prime}=0$. Contrasting (B.16) and (B.14) we infer $0=d Z_{0 X Y}-H_{1 X Y}^{\prime}$, which implies $Z_{0 X Y}=$ const and $H_{1 X Y}^{\prime}=0$. The constant $Z_{0 X Y}$ can be reabsorbed in $u_{X Y}$. 
After these simplifications, the deformations parametrized by $W_{3}$ and $\lambda_{I}$ take the form

$$
\begin{aligned}
& V_{4} \rightarrow V_{4}+d W_{3}, \\
& \omega_{I} \rightarrow \omega_{I}+\iota_{I} W_{3}+d \lambda_{I}, \quad \omega_{\alpha} \rightarrow \omega_{\alpha} \\
& \sigma_{I J} \rightarrow \iota_{(I} \lambda_{J)}, \quad \sigma_{I \alpha} \rightarrow 0, \quad \sigma_{\alpha \beta} \rightarrow 0 .
\end{aligned}
$$

This is equivalent to adding to the polyform $f_{V_{4}}(\mathcal{X})$ a $G$-equivariantly exact polyform,

$$
f_{V_{4}} \rightarrow f_{V_{4}}+d_{\text {eq }} f_{W_{3}}, \quad f_{W_{3}}(\mathcal{X})=W_{3}+\mathcal{X}^{I} \lambda_{I},
$$

while leaving the polyforms $f_{\omega_{\alpha}}$ unaffected.

If we focus instead on the deformation parametrized by $\lambda_{\alpha}$, we have

$$
\begin{aligned}
& V_{4} \rightarrow V_{4}, \\
& \omega_{I} \rightarrow \omega_{I}, \quad \omega_{\alpha} \rightarrow \omega_{\alpha}+d \lambda_{\alpha} \\
& \sigma_{I J} \rightarrow 0, \quad \sigma_{I \alpha} \rightarrow \frac{1}{2} \iota_{I} \lambda_{\alpha}, \quad \sigma_{\alpha \beta} \rightarrow 0 .
\end{aligned}
$$

In this case, the polyform $f_{V_{4}}$ is unaffected, while the polyform $f_{\omega_{\alpha}}$ are shifted by $G$ equivariantly exact terms,

$$
f_{\omega_{\alpha}} \rightarrow f_{\omega_{\alpha}}+d_{\mathrm{eq}} f_{\lambda_{\alpha}}, \quad f_{\lambda_{\alpha}}(\mathcal{X})=\lambda_{\alpha}
$$

Let us now discuss the deformation parametrized by $H_{2 I}$. Since this object is a harmonic 2-form, we must have

$$
H_{2 I}=c_{I}^{\alpha} \omega_{\alpha}
$$

with invariance under $G$ imposing $f_{I J}{ }^{K} c_{K}^{\alpha}=0$. This is a shift of $\omega_{I}$ by a combination of $\omega_{\alpha}$ 's, which is discussed in the main text around (3.23).

A deformation parametrized by $H_{2 \alpha}$ is a change of basis for the harmonic 2-forms, hence contains no interesting information.

Finally, constant shift by $u_{I J}, u_{I \alpha}$, are discussed around (3.23) and (3.26), while a shift by $u_{\alpha \beta}$ only affects the purely external, closed 4 -form $\gamma_{4}$.

\section{C (In)dependence of $I_{d+2}^{\text {inflow }}$ on $\gamma_{4}$}

Throughout this appendix we make use of the compact notation with collective index $X=(I, \alpha)$ introduced at the end of section 3.1. The most general $E_{4}$ in this language is given in (3.17), repeated here for convenience,

$$
E_{4}=V_{4}^{\mathrm{g}}+F^{X} \omega_{X}^{\mathrm{g}}+F^{X} F^{Y} \sigma_{X Y}+\gamma_{4} .
$$

Notice that in the main text we have set by definition $\sigma_{\alpha \beta}=0$. In this appendix, it is convenient to relax this assumption, and let $\sigma_{\alpha \beta}$ be an unspecified constant. Turning on $\sigma_{\alpha \beta}$ amounts to shifting $\gamma_{4}$ as

$$
\gamma_{4} \rightarrow \gamma_{4}+\frac{1}{(2 \pi)^{2}} F^{\alpha} F^{\beta} \sigma_{\alpha \beta}
$$

Since $\gamma_{4}$ is an arbitrary closed and gauge-invariant external 4 -form, this shift is immaterial.

The goal of this appendix is to analyze the dependence of $I_{d+2}^{\text {inflow }}=\int_{M_{10-d}} \mathcal{I}_{12}$ on $\gamma_{4}$ and on constant shifts in $\sigma_{X Y}$. 


\section{C.1 The case $d=6$}

Let us first focus on the contribution of the $E_{4}^{3}$ term in $\mathcal{I}_{12}$ to the inflow anomaly polynomial. Making use of the parametrization (3.17), we compute

$$
\begin{aligned}
-\frac{1}{6} \int_{M_{4}} E_{4}^{3}= & -\frac{1}{2} F^{X_{1}} F^{X_{2}} F^{X_{3}} F^{X_{4}} \int_{M_{4}}\left[\omega_{X_{1}} \omega_{X_{2}} \sigma_{X_{3} X_{4}}+V_{4} \sigma_{X_{1} X_{2}} \sigma_{X_{3} X_{4}}\right] \\
& -\frac{1}{2} F^{X} F^{Y} \gamma_{4} \int_{M_{4}}\left[\omega_{X} \omega_{Y}+2 V_{4} \sigma_{X Y}\right]-\frac{1}{2} \gamma_{4}^{2} \int_{M_{4}} V_{4} .
\end{aligned}
$$

Let us now turn to the term $E_{4} X_{8}$ in $\mathcal{I}_{12}$. We are only interested here in keeping track of terms with $\sigma_{X Y}, \gamma_{4}$. The quantity $E_{4} X_{8}$ is necessarily linear in these parameters. In order to have a $\sigma_{X Y}$ or $\gamma_{4}$ factor, we must select the part of $E_{4}$ with four external legs, which means that $X_{8}$ must saturate the integration over $M_{4}$. The relevant terms in $X_{8}$ can be written as

$$
X_{8}=Z V_{4}^{\mathrm{g}} p_{1}\left(T W_{6}\right)+Z_{X Y} V_{4}^{\mathrm{g}} F^{X} F^{Y}+\ldots,
$$

where $Z$ and $Z_{X Y}$ are constants. Notice that $X_{8}$ is not expected to receive any contribution proportional to the curvatures $F^{\alpha}$ associated to the harmonic 2-forms $\omega_{\alpha}$. As a result, the only non-zero components of $Z_{X Y}$ are $Z_{I J}$. Keeping nonetheless the collective indices $X$, $Y$, we have

$$
\begin{aligned}
-\int_{M_{4}} E_{4} X_{8}= & -\left[Z p_{1}\left(T W_{6}\right)+Z_{X Y} F^{X} F^{Y}\right] \int_{M_{4}}\left[\gamma_{4}+F^{X} F^{Y} \sigma_{X Y}\right] V_{4} \\
& + \text { terms without } \sigma_{X Y}, \gamma_{4} .
\end{aligned}
$$

We propose the following prescription to fix $\gamma_{4}$ : extremize $I_{8}^{\text {inflow }}$ with respect to arbitrary variations of $\gamma_{4}$. We compute

$$
\begin{aligned}
\delta I_{8}^{\text {inflow }}=\delta \gamma_{4}\{ & -\frac{1}{2} F^{X} F^{Y} \int_{M_{4}}\left[\omega_{X} \omega_{Y}+2 V_{4} \sigma_{X Y}\right] \\
& \left.-\left[\gamma_{4}+Z p_{1}\left(T W_{6}\right)+Z_{X Y} F^{X} F^{Y}\right] \int_{M_{4}} V_{4}\right\} .
\end{aligned}
$$

The quantity $\gamma_{4}$ is then be fixed to be

$$
\gamma_{4}=-\frac{1}{2} F^{X} F^{Y} \frac{\int_{M_{4}}\left(\omega_{X} \omega_{Y}+2 V_{4} \sigma_{X Y}\right)}{\int_{M_{4}} V_{4}}-Z p_{1}\left(T W_{6}\right)-Z_{X Y} F^{X} F^{Y} .
$$

To further elucidate the prescription (C.8), let us consider the 8-form $E_{4}^{2}+2 X_{8}$. The relevance of this combination stems from the fact that it corresponds to the combination $G_{4}^{2} /(2 \pi)^{2}+2 X_{8}$ that governs the M2-brane tadpole cancellation in M-theory compactifications. Let us focus on the part of $E_{4}^{2}+2 X_{8}$ with four legs on $M_{4}$,

$$
\left[E_{4}^{2}+2 X_{8}\right]_{4 \text { legs on } M_{4}}=F^{X} F^{Y}\left[\omega_{X} \omega_{Y}+2 V_{4} \sigma_{X Y}\right]+2 V_{4}\left[\gamma_{4}+Z p_{1}\left(T W_{6}\right)+Z_{X Y} F^{X} F^{Y}\right] \text {. }
$$


The r.h.s. is a sum of terms, each given by an external 4 -form wedge a 4 -form on $M_{4}$. Let us demand

$$
\left[E_{4}^{2}+2 X_{8}\right]_{4 \text { legs on } M_{4}}=0 \quad \text { in cohomology of } M_{4} \text {. }
$$

Since $h^{4}\left(M_{4}\right)=1$, the above requirement is equivalent to

$$
\int_{M_{4}}\left(E_{4}^{2}+2 X_{8}\right)=0
$$

Making use of (C.9), we see that this selects exactly the same $\gamma_{4}$ as in (C.8). This observation allows us to interpret the prescription (C.8) as an M2-brane tadpole cancellation condition.

\section{C.2 The case $d=4$}

In the case $d=4$, not all harmonic 2-forms $\omega_{\alpha}$ are associated to global symmetries of the setup. To clarify this point, let us consider the low-energy effective action for the compactification of M-theory on the internal space $M_{6}$. Assuming supersymmetry is not completely broken, the low-energy theory is a supergravity theory in five dimensions. One linear combination of the vectors $A^{\alpha}$ associated to $\omega_{\alpha}$ gets massive because of its coupling to a $5 \mathrm{~d}$ axion.

The 11d background metric for the compactification is of the form

$$
d s^{2}\left(M_{11}\right)=e^{2 \lambda} d s^{2}\left(W_{5}\right)+d s^{2}\left(M_{6}\right),
$$

where $\lambda$ is a warp factor and $W_{5}$ denotes the $5 \mathrm{~d}$ spacetime where the low-energy supergravity is defined. We refrain from a full analysis of the low-energy dynamics, and only focus on the relevant couplings. The $G_{4}$-flux consists of a background part, together with fluctuations. Let us write

$$
\frac{G_{4}}{2 \pi}=V_{4}^{\mathrm{g}}+F^{X} \omega_{X}^{\mathrm{g}}+F^{X} F^{Y} \sigma_{X Y}+g_{4}+\ldots
$$

In the previous expression, $V_{4}$ is the $G_{4}$-flux configuration in the background. The gauging procedure couples it to the $5 \mathrm{~d}$ vectors associated to isometries of $M_{6}$. The term $F^{X} \omega_{X}^{\mathrm{g}}$ contains both the vectors associated to isometries, and the vectors associated to harmonic 2-forms $\omega_{\alpha}$. The term $F^{X} F^{Y} \sigma_{X Y}$ is higher-order in external fluctuations, but we have included because it is needed for closure of $G_{4}$. Finally, $g_{4}$ is a $5 \mathrm{~d}$ field, independent of the internal coordinates. It is the zeromode in the Kaluza-Klein expansion of $G_{4}$ onto scalar harmonics on $M_{6}$. This $5 \mathrm{~d}$ field satisfies

$$
d g_{4}=0, \quad g_{4}=d c_{3}
$$

with $c_{3}$ a 3 -form potential in five dimensions. Notice that a 3 -form potential in five dimensions is dual to a 0 -form potential, i.e. an axion.

The topological couplings in the 11d action induce Chern-Simons couplings in the lowenergy 5d supergravity theory. A convenient way to perform the dimensional reduction is to write the Chern-Simons interactions in one dimension higher. We thus introduce 
$M_{12}$ with $\partial M_{12}=M_{11}$, as well as $W_{6}$ with $\partial W_{6}=W_{5}$. The $C_{3} G_{4} G_{4}$ term in $M_{11}$ is reformulated as $G_{4} G_{4} G_{4}$ in $M_{12}$, and upon reduction on $M_{6}$ yields the couplings

$$
\int_{M_{6}}-\frac{1}{6}\left[\frac{G_{4}}{2 \pi}\right]^{3}=-\frac{1}{2} g_{4} F^{X} \int_{M_{6}} V_{4} \omega_{X}-\frac{1}{6} F^{X} F^{Y} F^{Z} \int_{M_{6}}\left(\omega_{X} \omega_{Y} \omega_{Z}+3 V_{4} \omega_{X} \sigma_{Y Z}\right) .
$$

The second coupling is a Chern-Simons coupling in five dimensions, and is not instrumental for our analysis. The first coupling is the essential ingredient in what follows.

Recall from the discussion around (3.25) that we are free to shift $\omega_{I}$ with $\omega_{\alpha}$ 's, if we perform a compensating redefinition of the curvatures $F^{\alpha}$. In particular, we can always shift the forms $\omega_{I}$ with linear combinations of $\omega_{\alpha}$ in such a way as to obtain

$$
\int_{M_{6}} V_{4} \omega_{I}=0
$$

With this choice of basis, $g_{4}$ is only coupling to the vectors $F^{\alpha}$ associated to the harmonic 2-forms $\omega_{\alpha}$.

The considerations of the previous paragraph show that the terms in the $5 \mathrm{~d}$ low-energy effective action involving $g_{4}$ are

$$
S_{5 \mathrm{~d}}=\int_{W_{5}}\left[-\frac{1}{2} \mathcal{G} g_{4} * g_{4}-\mathcal{K}_{\alpha} g_{4} A^{\alpha}+\ldots\right], \quad \mathcal{K}_{\alpha}=\frac{1}{4 \pi} \int_{M_{6}} V_{4} \omega_{\alpha} .
$$

The Hodge star is computed with the external metric on $W_{5}$. The quantity $\mathcal{G}$ in the kinetic term for $g_{4}$ can be fixed by reducing $G_{4} *_{11} G_{4}$ on the background (C.12). We do not need the precise expression of $\mathcal{G}$ in what follows. In the action (C.17), $g_{4}$ is the field strength of the 3 -form potential $c_{3}$, which is regarded as dynamical field. We can alternatively dualize, by adding a 0 -form Lagrange multiplier $\Phi$ to impose the Bianchi identity for $g_{4}$,

$$
\Delta S_{5 \mathrm{~d}}=-\int_{W_{5}} g_{4} d \Phi
$$

If we eliminate $g_{4}$ using its equation of motion, we obtain

$$
S_{5 \mathrm{~d}}+\Delta S_{5 \mathrm{~d}}=\int_{W_{5}}-\frac{1}{2 \mathcal{G}} D \Phi * D \Phi+\ldots, \quad D \Phi=d \Phi+\mathcal{K}_{\alpha} A^{\alpha}
$$

The scalar $\Phi$ has a shift symmetry coupled to the combination $\mathcal{K}_{\alpha} A^{\alpha}$, which is thus rendered massive, as anticipated.

In the computation of the inflow anomaly polynomial, the connections $A^{I}, A^{\alpha}$ are background fields coupled to the global symmetries of the system. We have argued that the combination $\mathcal{K}_{\alpha} A^{\alpha}$ does not correspond to a symmetry. As a result, we must set it to zero in the computation of the anomaly,

$$
F^{\alpha} \int_{M_{6}} V_{4} \omega_{\alpha}=0 .
$$

Since we work in a basis such that (C.16) holds, the condition (C.20) is equivalent to

$$
F^{X} \int_{M_{6}} V_{4} \omega_{X}=0 .
$$


We are now in a position to analyze how ambiguities in $E_{4}$ affect the inflow anomaly polynomial. First of all, let us study the $E_{4}^{3}$ term in $\mathcal{I}_{12}$. We compute

$$
-\frac{1}{6} \int_{M_{6}} E_{4}^{3}=-\frac{1}{6} F^{X} F^{Y} F^{Z} \int_{M_{6}}\left[\omega_{X} \omega_{Y} \omega_{Z}+6 V_{4} \omega_{X} \sigma_{Y Z}\right]-F^{X} \gamma_{4} \int_{M_{6}} V_{4} \omega_{X} .
$$

The dependence on $\gamma_{4}$ immediately drops away thanks to (C.21). The same holds true for any dependence on shifts of $\sigma_{X Y}$. Indeed, we can write

$$
\sigma_{X Y}=\bar{\sigma}_{X Y}+u_{X Y}
$$

where $\bar{\sigma}_{X Y}$ is a reference choice for $\sigma_{X Y}$, and $u_{X Y}$ are arbitrary constant. The dependence on $u_{X Y}$ in (C.22) disappears, thanks to

$$
-\frac{1}{6} F^{X} F^{Y} F^{Z} \int_{M_{6}} 6 V_{4} \omega_{X} u_{Y Z}=-u_{Y Z} F^{Y} F^{Z}\left(F^{X} \int_{M_{6}} V_{4} \omega_{X}\right)=0 .
$$

In conclusion, the value of $\int_{M_{6}} E_{4}^{3}$ is insensitive to ambiguities in $E_{4}$.

The term $E_{4} X_{8}$ can be sensitive to ambiguities in $E_{4}$ if $X_{8}$ can saturate the integral over $M_{6}$. For this to be possible, $X_{8}$ must contain a term of the form

$$
X_{8}=Z_{6 I}^{\mathrm{g}} F^{I}+\ldots,
$$

where $Z_{6 I}$ is a 6 -form on $M_{6}$ and the label $I$ refers to a $\mathrm{U}(1)$ factor in the isometry group. In all examples we consider in this work, however, $X_{8}$ does not contain any terms of the form (C.25). While we do not have a general proof, we suspect that this feature should hold in general. As a result, the term $E_{4} X_{8}$ is insensitive to ambiguities in $E_{4}$, and the full inflow anomaly polynomial is determined unambiguously.

\section{C.3 The case $d=2$}

The contribution to the inflow anomaly polynomial coming from the $E_{4}^{3}$ term in $\mathcal{I}_{12}$ reads

$$
-\frac{1}{6} \int_{M_{8}} E_{4}^{3}=-\frac{1}{2} F^{X} F^{Y} \int_{M_{8}}\left[V_{4} \omega_{X} \omega_{Y}+V_{4}^{2} \sigma_{X Y}\right]-\frac{1}{2} \gamma_{4} \int_{M_{8}} V_{4}^{2} .
$$

As far as the $E_{4} X_{8}$ term is concerned, it can depend on ambiguities in $E_{4}$ only if $X_{8}$ can saturate the integration in the internal space $M_{8}$. The part of $X_{8}$ with 8 internal legs is the 8 -form

$$
Z_{8}=X_{8}^{\text {background }}=\frac{1}{192}\left[p_{1}\left(T M_{8}\right)^{2}-4 p_{2}\left(T M_{8}\right)\right]
$$

where the label "background" refers to the fact that $Z_{8}$ is the value of $X_{8}$ when all external curvatures are turned off. The terms in $E_{4} X_{8}$ that are potentially ambiguous are then

$$
-\int_{M_{8}} E_{4} X_{8}=-F^{X} F^{Y} \int_{M_{8}} Z_{8} \sigma_{X Y}-\gamma_{4} \int_{M_{8}} Z_{8}+\ldots
$$

In summary, the terms in the inflow anomaly polynomial containing $\gamma_{4}$ or $\sigma_{X Y}$ are

$$
I_{4}^{\text {inflow }}=-\frac{1}{2} F^{X} F^{Y} \int_{M_{8}}\left(V_{4}^{2}+2 Z_{8}\right) \sigma_{X Y}-\frac{1}{2} \gamma_{4} \int_{M_{8}}\left(V_{4}^{2}+2 Z_{8}\right)+\ldots
$$


We argue, however, that a good M-theory background necessarily requires

$$
\int_{M_{8}}\left(V_{4}^{2}+2 Z_{8}\right)=0
$$

This condition is the tadpole cancellation condition that must hold for any compactification of M-theory on an 8-manifold in absence of localized M2-brane sources. As we can see, thanks to (C.30) the inflow anomaly polynomial (C.29) is independent on $\gamma_{4}$ and on constant shifts of $\sigma_{X Y}$. As a result, it is completely determined.

\section{Details on branes at an orbifold singularity}

Preliminaries. When the M5-brane stack probes an orbifold singularity, the isometry of $S^{4}$ is reduced to a subgroup of $\mathrm{SU}(2)_{L} \times \mathrm{SU}(2)_{R}$ of $\mathrm{SO}(5)$. Under the reduction $\mathrm{SO}(5) \rightarrow$ $\mathrm{SU}(2)_{L} \times \mathrm{SU}(2)_{R}$, we find

$$
\begin{aligned}
p_{1}(\mathrm{SO}(5)) & =-2\left[c_{2}(L)-c_{2}(R)\right], \\
p_{2}(\mathrm{SO}(5)) & =\left[c_{2}(L)-c_{2}(R)\right]^{2}, \\
F^{A B} F^{C D} \bar{\sigma}_{A B, C D} & =\frac{1}{2} y^{5} N|\Gamma|\left[c_{2}(L)-c_{2}(R)\right] .
\end{aligned}
$$

In the above expressions $c_{2}(L, R) \equiv c_{2}\left(\mathrm{SU}(2)_{L, R}\right)$. We have used the expression for $\bar{\sigma}_{A B, C D}$ in (4.5), keeping in mind that $N$ is now replaced by $N|\Gamma|$.

The quantity $c_{2}(L)$ contains both internal and external contributions. The external contribution is related to isometries of $S^{4} / \Gamma$, and is only present if $\Gamma$ is of A type. The internal contributions are present for any $\Gamma$ and are localized at the north and south poles. They measure the curvature of the ALE spaces that resolve the orbifold singularities $\mathbb{C}^{2} / \Gamma$ at each pole. Since the two singularities are identical,

$$
\int_{\mathrm{N}} c_{2}(L)=\int_{\mathrm{S}} c_{2}(L)=\chi_{\Gamma}
$$

In the above relations the symbols $\int_{\mathrm{N}, \mathrm{S}}$ denote schematically integration on the ALE space at the north, south pole respectively. The Euler characteristic $\chi_{\Gamma}$ is given in (4.14).

The form $\boldsymbol{E}_{4}$. The 4-form $E_{4}$ is given in (4.12), repeated here for convenience,

$$
E_{4}=\bar{V}_{4}^{\mathrm{g}}+F^{A B} \bar{\omega}_{A B}^{\mathrm{g}}+F^{A B} F^{C D} \bar{\sigma}_{A B, C D}+\frac{F^{\mathrm{N} i}}{2 \pi} \omega_{\mathrm{N} i}+\frac{F^{\mathrm{S} i}}{2 \pi} \omega_{\mathrm{S} i}+\gamma_{4} .
$$

It is important to stress that all curvatures in $E_{4}$, including the curvature of $\mathrm{SU}(2)_{L}$, are understood to have purely external legs. In other words, the term $F^{A B} F^{C D} \bar{\sigma}_{A B, C D}$ does not contain the internal part of $c_{2}(L)$ that integrates to $\chi_{\Gamma}$ at the ALE spaces near the poles. This observation is crucial to obtain the correct result. The fact that $E_{4}$ only contains the external connections is due to the fact that it is built gauging the isometries of $S^{4} / \Gamma$. 
The form $\boldsymbol{X}_{\mathbf{8}}$. The Pontryagin classes of the total space can be written as

$$
\begin{aligned}
& p_{1}\left(T M_{11}\right)=p_{1}\left(T W_{6}\right)+p_{1}(\mathrm{SO}(5)), \\
& p_{2}\left(T M_{11}\right)=p_{2}\left(T W_{6}\right)+p_{2}(\mathrm{SO}(5))+p_{1}\left(T W_{6}\right) p_{1}(\mathrm{SO}(5)),
\end{aligned}
$$

where $p_{1,2}(\mathrm{SO}(5))$ are given as in (D.1). Expressing $X_{8}$ in terms of $p_{1,2}\left(T W_{6}\right), c_{2}(L, R)$, we arrive at

$$
X_{8}=\frac{1}{48} c_{2}(L)\left[p_{1}\left(T W_{6}\right)+4 c_{2}(R)\right]+\frac{1}{192}\left[p_{1}\left(T W_{6}\right)^{2}-4 p_{2}\left(T W_{6}\right)\right]+\frac{1}{48} c_{2}(R) p_{1}\left(T W_{6}\right) .
$$

Let us stress that here $c_{2}(L)$ contains both internal and external parts, while all other curvatures have legs along $W_{6}$ only.

Integral of $\boldsymbol{E}_{\mathbf{4}} \boldsymbol{X}_{\mathbf{8}}$. The 4-form $E_{4}$ is given in (4.12). The integral $\int_{M_{4}} E_{4} X_{8}$ receives two types of contributions. Firstly, we can consider the purely external part of $X_{8}$, and saturate the integral over $S^{4}$ using the part of $E_{4}$ with four internal legs, which is $\bar{V}_{4}$. This contribution is

$$
\begin{aligned}
\int_{M_{4}} E_{4} X_{8} \supset \frac{N|\Gamma|}{|\Gamma|}\left\{\frac{1}{48} c_{2}(L)^{\operatorname{ext}}\left[p_{1}\left(T W_{6}\right)+4 c_{2}(R)\right]+\frac{1}{192}\left[p_{1}\left(T W_{6}\right)^{2}-4 p_{2}\left(T W_{6}\right)\right]\right. \\
\left.+\frac{1}{48} c_{2}(R) p_{1}\left(T W_{6}\right)\right\}
\end{aligned}
$$

where the factor $N|\Gamma|$ originates from the new normalization of $\bar{V}_{4}$, the factor $1 /|\Gamma|$ originates from the integral over $S^{4} / \Gamma$ (as opposed to $S^{4}$ ). The superscript "ext" on $c_{2}(L)$ denotes its external part.

The other contribution to $\int_{M_{4}} E_{4} X_{8}$ is obtained by saturating the integration over $M_{4}$ with the internal part of $c_{2}(L)$ inside $X_{8}$. We then consider the purely external part of $E_{4}$. We find

$$
\int_{M_{4}} E_{4} X_{8} \supset \frac{1}{48}\left[p_{1}\left(T W_{6}\right)+4 c_{2}(R)\right] \int_{M_{4}} c_{2}(L)\left[F^{A B} F^{C D} \bar{\sigma}_{A B, C D}+\gamma_{4}\right] .
$$

The integral over $M_{4}$ localizes at the two poles. More precisely, we get integrals over the ALE spaces that resolve the orbifold singularities at each pole. Taking into account the opposite orientation of the two poles, we can write

$$
\begin{aligned}
\int_{M_{4}} c_{2}(L)\left[F^{A B} F^{C D} \bar{\sigma}_{A B, C D}+\gamma_{4}\right]= & \int_{\mathrm{N}} c_{2}(L)\left[F^{A B} F^{C D} \bar{\sigma}_{A B, C D}+\gamma_{4}\right]^{\mathrm{N}} \\
& -\int_{\mathrm{S}} c_{2}(L)\left[F^{A B} F^{C D} \bar{\sigma}_{A B, C D}+\gamma_{4}\right]^{\mathrm{S}} .
\end{aligned}
$$

Since $\gamma_{4}$ is independent on the coordinates on $S^{4}$, it drops away. In contrast, $F^{A B} F^{C D} \bar{\sigma}_{A B, C D}^{\mathrm{N}}=-F^{A B} F^{C D} \bar{\sigma}_{A B, C D}^{\mathrm{S}}$, and the two terms add to

$$
\int_{M_{4}} c_{2}(L)\left[F^{A B} F^{C D} \bar{\sigma}_{A B, C D}+\gamma_{4}\right]=N|\Gamma| \chi_{\Gamma}\left[c_{2}(L)^{\mathrm{ext}}-c_{2}(R)\right],
$$

where we have used (D.1) and (D.2). 
In conclusion, the integral of $E_{4} X_{8}$ is given by

$$
\begin{aligned}
\int_{M_{4}} E_{4} X_{8}= & \frac{N|\Gamma| \chi_{\Gamma}}{48}\left[c_{2}(L)^{\mathrm{ext}}-c_{2}(R)\right]\left[p_{1}\left(T W_{6}\right)+4 c_{2}(R)\right]+\frac{N}{48} c_{2}(L)^{\mathrm{ext}}\left[p_{1}\left(T W_{6}\right)+4 c_{2}(R)\right] \\
& +\frac{N}{192}\left[p_{1}\left(T W_{6}\right)^{2}-4 p_{2}\left(T W_{6}\right)\right]+\frac{N}{48} c_{2}(R) p_{1}\left(T W_{6}\right) .
\end{aligned}
$$

Integral of $\boldsymbol{E}_{4}^{\mathbf{3}}$. A first set of contributions to $\int_{M_{4}} E_{4}^{3}$ originates from the region away from the north and south poles. These contributions are given by

$$
\frac{1}{6} \int_{M_{4}} E_{4}^{3} \supset \int_{S^{4} / \Gamma}\left[\frac{1}{2}(F F \bar{\sigma})(F \bar{\omega})^{2}+\frac{1}{2}(F F \bar{\sigma})^{2} \bar{V}_{4}+\frac{1}{2}(F \bar{\omega})^{2} \gamma_{4}+(F F \bar{\sigma}) \bar{V}_{4} \gamma_{4}+\frac{1}{2} \bar{V}_{4} \gamma_{4}^{2}\right],
$$

where we are suppressing $\mathrm{SO}(5)$ indices for brevity. The integral over $S^{4} / \Gamma$ can be computed with the identities (A.17), (A.18). We must keep in mind, however, that the quotient by $\Gamma$ generates an additional factor $1 /|\Gamma|$. We then verify that the r.h.s. of (D.9) is equal to

$$
\frac{N^{3}|\Gamma|^{2}}{24} p_{2}(\mathrm{SO}(5))+\frac{1}{2} N \gamma_{4}^{2}
$$

Let us now discuss the contributions to $\int_{M_{4}} E_{4}^{3}$ coming from the harmonic 2-forms localized at the north and south poles. These terms are

$$
\frac{1}{6} \int_{M_{4}} E_{4}^{3} \supset \frac{1}{2} \int_{M_{4}}\left[(F F \bar{\sigma})+\gamma_{4}\right]\left[\frac{\left(F^{\mathrm{N} i} \omega_{\mathrm{N} i}\right)^{2}}{(2 \pi)^{2}}+\frac{\left(F^{\mathrm{S} i} \omega_{\mathrm{S} i}\right)^{2}}{(2 \pi)^{2}}\right]
$$

To proceed, we make use of

$$
\begin{aligned}
\int_{M_{4}}\left(F F \bar{\sigma}+\gamma_{4}\right) \frac{\left(F^{\mathrm{N}} \omega_{\mathrm{N}}\right)^{2}}{(2 \pi)^{2}} & =+\left(F F \bar{\sigma}^{\mathrm{N}}+\gamma_{4}\right) \frac{F^{\mathrm{N} i}}{2 \pi} \frac{F^{\mathrm{N} j}}{2 \pi} \int_{\mathrm{N}} \omega_{\mathrm{N} i} \omega_{\mathrm{N} j} \\
\int_{M_{4}}\left(F F \bar{\sigma}+\gamma_{4}\right) \frac{\left(F^{\mathrm{S}} \omega_{\mathrm{S}}\right)^{2}}{(2 \pi)^{2}} & =-\left(F F \bar{\sigma}^{\mathrm{S}}+\gamma_{4}\right) \frac{F^{\mathrm{S} i}}{2 \pi} \frac{F^{\mathrm{S} j}}{2 \pi} \int_{\mathrm{N}} \omega_{\mathrm{S} i} \omega_{\mathrm{S} j} .
\end{aligned}
$$

The relative sign is due to the different orientation of the two ALE spaces near the north and south poles. The integral $\int_{\mathrm{N}} \omega_{\mathrm{N} i} \omega_{\mathrm{N} j}$ is proportional to the entries of the Cartan matrix of the ADE Lie algebra $\mathfrak{g}_{\Gamma}$ associated to $\Gamma$, and similarly for $\int_{\mathrm{N}} \omega_{\mathrm{S} i} \omega_{\mathrm{S} j}$. We know that, at the conformal point where all resolution $\mathbb{C P}^{1}$ 's are shrunk to zero size, we have a nonAbelian enhancement of the flavor symmetry at each pole. In light of this observation, we make the replacements

$$
\frac{F^{\mathrm{N} i}}{2 \pi} \frac{F^{\mathrm{N} j}}{2 \pi} \int_{\mathrm{N}} \omega_{\mathrm{N} i} \omega_{\mathrm{N} j} \rightarrow \frac{1}{2} \frac{\operatorname{tr}\left(F^{\mathrm{N}}\right)^{2}}{(2 \pi)^{2}}, \quad \frac{F^{\mathrm{S} i}}{2 \pi} \frac{F^{\mathrm{S} j}}{2 \pi} \int_{\mathrm{S}} \omega_{\mathrm{S} i} \omega_{\mathrm{S} j} \rightarrow \frac{1}{2} \frac{\operatorname{tr}\left(F^{\mathrm{S}}\right)^{2}}{(2 \pi)^{2}} .
$$

Recalling (D.1), it follows that the r.h.s. of (D.11) is equal to

$$
\frac{N|\Gamma|}{8}\left[c_{2}(L)^{\mathrm{ext}}-c_{2}(R)\right]\left[\frac{\operatorname{tr}\left(F^{\mathrm{N}}\right)^{2}}{(2 \pi)^{2}}+\frac{\operatorname{tr}\left(F^{\mathrm{S}}\right)^{2}}{(2 \pi)^{2}}\right]+\frac{1}{4} \gamma_{4}\left[\frac{\operatorname{tr}\left(F^{\mathrm{N}}\right)^{2}}{(2 \pi)^{2}}-\frac{\operatorname{tr}\left(F^{\mathrm{S}}\right)^{2}}{(2 \pi)^{2}}\right] .
$$


In conclusion, the integral of $E_{4}^{3}$ yields

$$
\begin{aligned}
\frac{1}{6} \int_{M_{4}} E_{4}^{3}= & \frac{N^{3}|\Gamma|^{2}}{24}\left[c_{2}(L)^{\text {ext }}-c_{2}(R)\right]^{2}+\frac{1}{2} N \gamma_{4}^{2} \\
& +\frac{N|\Gamma|}{8}\left[c_{2}(L)^{\mathrm{ext}}-c_{2}(R)\right]\left[\frac{\operatorname{tr}\left(F^{\mathrm{N}}\right)^{2}}{(2 \pi)^{2}}+\frac{\operatorname{tr}\left(F^{\mathrm{S}}\right)^{2}}{(2 \pi)^{2}}\right] \\
& +\frac{1}{4} \gamma_{4}\left[\frac{\operatorname{tr}\left(F^{\mathrm{N}}\right)^{2}}{(2 \pi)^{2}}-\frac{\operatorname{tr}\left(F^{\mathrm{S}}\right)^{2}}{(2 \pi)^{2}}\right] .
\end{aligned}
$$

The sum of the above quantity with $\int_{M_{4}} E_{4} X_{8}$ given in (D.8) gives $-I_{8}^{\text {inflow }}$ as quoted in the main text in (4.13).

\section{E Details on the BBBW setup}

This appendix is devoted to some derivations regarding the setups studied in section 5.1. The relevant space $M_{6}$ is an $S^{4}$ fibration over $\Sigma_{g}$, specified by the background flux (5.3), repeated here for the reader's convenience,

$$
F_{\Sigma}^{A B}=q^{A B} V_{\Sigma}, \quad \int_{\Sigma_{g}} V_{\Sigma}=2 \pi .
$$

The matrix $q^{A B}$ is given in (5.3). All the following results, however, hold for any constant antisymmetric $q^{A B}$.

Additional isometries in the case $\boldsymbol{g}=\mathbf{0}$. In the case $g=0$, the line element of $M_{6}$ reads

$$
d s^{2}\left(M_{6}\right)=d s^{2}\left(S^{2}\right)+d s^{2}\left(S^{4}\right)^{\mathrm{t}}=d s^{2}\left(S^{2}\right)+\left(d y^{A}-q^{A B} y_{B} \mathcal{V}\right)\left(d y_{A}-q_{A C} y^{C} \mathcal{V}\right) .
$$

Recall that $y^{A}, A=1, \ldots, 5$ are constrained coordinates for the $S^{4}$ fiber, $y^{A} y_{A}=1$. The 1-form $\mathcal{V}$ is defined only locally, and is an antiderivative of $V_{\Sigma}$,

$$
d \mathcal{V}=V_{\Sigma}
$$

We find it convenient to parametrize the base $S^{2}$ in terms of three constrained coordinates $z^{a} z_{a}=1, a=1,2,3$. By means of a direct computation, one verifies that the following triplet of 1 -forms on $M_{6}$ are such that the dual contravariant vectors are Killing,

$$
k_{a}=\epsilon_{a b c} z^{b} d z^{c}-\frac{1}{2} z_{a} q^{A B} y_{A}\left(d y^{B}-q^{B C} y_{C} \mathcal{V}\right)
$$

The term $\frac{1}{2} \epsilon_{a b c} z^{b} d z^{c}$ is the expression of the Killing 1-forms of a round $S^{2}$ considered in isolation. The other terms in (E.4) demonstrate how these 1-forms are extended to the total space $M_{6}$, depending on the twist data $q^{A B}$.

The explicit expression (E.4) of the Killing 1-forms $k_{a}$ is useful in checking the following identities,

$$
\begin{aligned}
\iota_{a}\left(d y^{A}\right)^{\mathrm{t}} & =\frac{1}{2} z_{a} q^{A B} y_{B}, & \iota_{a} d z^{b} & =-\epsilon_{a}^{b c} z_{c} \\
V_{\Sigma} & =\frac{1}{2} \cdot \frac{1}{2} \epsilon_{a b c} d z^{a} d z^{b} z^{c}, & \iota_{a} V_{\Sigma} & =\frac{1}{2} d z_{a} .
\end{aligned}
$$


We can now compute the interior product of $k_{a}$ with $V_{4}$ given in (5.8). Two useful partial results are

$$
\begin{aligned}
\iota_{a}\left(\bar{V}_{4}\right)^{\mathrm{t}} & =\frac{3 N}{8 \pi^{2}} \cdot \frac{1}{12} z_{a} \epsilon_{A B C D E}\left(q^{A A^{\prime}} y_{A^{\prime}}\right)\left(d y^{B}\right)^{\mathrm{t}}\left(d y^{C}\right)^{\mathrm{t}}\left(d y^{D}\right)^{\mathrm{t}} y^{E} \\
& =\frac{3 N}{8 \pi^{2}} \cdot \frac{-1}{24} z_{a} \epsilon_{A B C D E} q^{A B}\left(d y^{C}\right)^{\mathrm{t}}\left(d y^{D}\right)^{\mathrm{t}}\left(d y^{E}\right)^{\mathrm{t}} \\
q^{A B} \iota_{a} \bar{\omega}_{A B}^{\mathrm{t}} & =\frac{3 N}{8 \pi^{2}} \cdot \frac{-1}{12} z_{a} \epsilon_{A B C D E} q^{A B}\left(q^{C C^{\prime}} y_{C^{\prime}}\right)\left(d y^{D}\right)^{\mathrm{t}} y^{E} \\
& =\frac{3 N}{8 \pi^{2}} \cdot \frac{1}{48} z_{a} \epsilon_{A B C D E} q^{A B} q^{C D}\left(d y^{E}\right)^{\mathrm{t}},
\end{aligned}
$$

where we used two Schouten identities deriving from $\delta_{B\left[A_{1}\right.} \epsilon_{\left.A_{2} A_{3} A_{4} A_{5} A_{6}\right]}=0$. Combining all elements, we verify the identity

$$
\begin{aligned}
\iota_{a} V_{4} & =\frac{3 N}{8 \pi^{2}} \cdot \frac{1}{24} d\left\{z_{a}\left[V_{\Sigma} \epsilon_{A B C D E} q^{A B} q^{C D} y^{E}-\epsilon_{A B C D E} q^{A B}\left(d y^{C}\right)^{\mathrm{t}}\left(d y^{D}\right)^{\mathrm{t}} y^{E}\right]\right\} \\
& =d\left\{z_{a}\left[V_{\Sigma} q^{A B} q^{C D} \bar{\sigma}_{A B, C D}+\frac{1}{2} q^{A B} \bar{\omega}_{A B}^{\mathrm{t}}\right]\right\} .
\end{aligned}
$$

Derivation of $\boldsymbol{E}_{4}$. The 4-form $E_{4}$ is constructed as

$$
E_{4}=V_{4}^{\mathrm{g}}+F^{I} \omega_{I}^{\mathrm{g}}+F^{I} F^{J} \sigma_{I J}+C p_{1}\left(T W_{4}\right) .
$$

Here we have used (3.17), combined with the observation that $M_{6}$ has no harmonic 2forms, so that the collective index $X$ reduces to the isometry label $I$. The latter refers both to isometries of class (i) and isometries of class (ii), in the terminology of section 5.1. Accordingly, we split the $I$ index as

$$
I=(\hat{I}, a), \quad \hat{I}=1,2, \quad a=1,2,3 .
$$

We have already introduced the $\mathrm{SO}(3)_{S^{2}}$ index $a$ above. The new index $\hat{I}$ refers to isometries of class (i). More precisely, we describe the external connections $A_{\text {ext }}^{A B}$ of (5.5) by writing

$$
A_{\mathrm{ext}}^{A B}=A^{\hat{I}} M_{\hat{I}}^{A B}
$$

where the index $\hat{I}$ labels the two generators of the class (i) isometry $\mathrm{SO}(2)_{1} \times \mathrm{SO}(2)_{2}$, $A^{\hat{I}}=\left(A_{1}, A_{2}\right)$. The matrices $M_{\hat{I}}^{A B}$ are constant and readily read off from (5.5),

$$
M_{1}{ }^{A B}=\left(\begin{array}{ccccc}
0 & -1 & & \\
1 & 0 & & \\
& & 0 & \\
& & 0 & \\
& & & 0
\end{array}\right), \quad M_{2}{ }^{A B}=\left(\begin{array}{ccccc}
0 & & & \\
0 & & & \\
& 0 & -1 & \\
& 1 & 0 & \\
& & & & 0
\end{array}\right) .
$$

The Killing vectors associated to $A^{\hat{I}}$ are linear combinations of the Killing vectors $k_{A B}$ of the round $S^{4}$,

$$
k_{\hat{I}}=M_{\hat{I}}^{A B} k_{A B},
$$

with $k_{A B}$ as in (A.20). 
Determining $E_{4}$ amounts to solving the following equations for $\omega_{I}, \sigma_{I J}$,

$$
\iota_{I} V_{4}+d \omega_{I}=0, \quad \iota_{(I} \omega_{J)}+d \sigma_{I J}=0
$$

where $I=(\hat{I}, a)$ and $V_{4}$ is given in (5.8).

Solution for $\omega_{I}$. Let us first discuss the forms $\omega_{\hat{I}}$ associated to isometries from the $S^{4}$ fiber. A natural ansatz for $\omega_{\hat{I}}$ is

$$
\omega_{\hat{I}}=\widetilde{\omega}_{\hat{I}}^{\mathrm{t}}+V_{\Sigma} g_{\hat{I}}
$$

where $\widetilde{\omega}_{\hat{I}}$ is a 2 -form with two legs along the $S^{4}$ fibers, while $g_{\hat{I}}$ are 0 -forms. The equation that determines $\omega_{\hat{I}}$ is $d \omega_{\hat{I}}+\iota_{\hat{I}} V_{4}=0$. Upon using (E.14) and separating terms with and without $V_{\Sigma}$, we find the relations

$$
d \widetilde{\omega}_{\hat{I}}+\iota_{\hat{I}} \bar{V}_{4}=0, \quad d g_{\hat{I}}+p^{A B} \iota_{A B} \widetilde{\omega}_{\hat{I}}+q^{A B} \iota_{\hat{I}} \bar{\omega}_{A B}=0 .
$$

The first equation is readily solved by setting

$$
\widetilde{\omega}_{\hat{I}}=M_{\hat{I}}^{A B} \bar{\omega}_{A B}
$$

Since by assumption $\widetilde{\omega}_{I}$ is a 2 -form in the $S^{4}$ fiber, there is no non-trivial closed but not exact form that we can add to it. Adding an exact piece would have no effect on the inflow anomaly polynomial. The second equation in (E.15) becomes

$$
0=d g_{\hat{I}}+q^{A B} M_{\hat{I}}^{C D}\left(\iota_{A B} \bar{\omega}_{C D}+\iota_{C D} \bar{\omega}_{A B}\right)=d g_{\hat{I}}-2 q^{A B} M_{\hat{I}}^{C D} d \bar{\sigma}_{A B, C D},
$$

where we have used (A.22). As we can see, $g_{\hat{I}}$ is fixed up to a constant. More precisely, the second equation in (E.15) only has to hold when wedged with $V_{\Sigma}$. In summary, $\omega_{\hat{I}}$ is given by

$$
\omega_{\hat{I}}=M_{\hat{I}}^{A B} \bar{\omega}_{A B}^{\mathrm{t}}+2 V_{\Sigma} q^{A B} M_{\hat{I}}^{C D} \bar{\sigma}_{A B, C D}+C_{\hat{I}} V_{\Sigma},
$$

where $C_{\hat{I}}$ are arbitrary functions depending on $\Sigma$ only. The term $C_{\hat{I}} V_{\Sigma}$ is thus closed but not necessarily exact. We can be more precise: since $C_{\hat{I}} V_{\Sigma}$ is a closed form on $S^{2}$, it can be decomposed as a sum of an exact form and a harmonic form. The exact piece can be disregarded. The harmonic piece must be a constant multiple of $V_{\Sigma}$. It follows that, without any loss of generality, we can take $C_{\hat{I}}$ to be constant.

In the case $g=0$ we also have to construct $\omega_{a}$, which must satisfy $d \omega_{a}+\iota_{a} V_{4}=0$. Thanks to (E.7), we know how to write $\iota_{a} V_{4}$ as a total derivative. As a result, $\omega_{a}$ is given by

$$
\omega_{a}=-z_{a}\left[V_{\Sigma} q^{A B} q^{C D} \bar{\sigma}_{A B, C D}+\frac{1}{2} q^{A B} \bar{\omega}_{A B}^{\mathrm{t}}\right]+C_{a} V_{\Sigma}
$$

Once again, we have not included an exact piece, because it would have no effect on the inflow anomaly polynomial. A priori, the 0 -form $C_{a}$ is allowed to have an arbitrary dependence on $S^{2}$. Using arguments similar to those of the previous paragraphs, however, we argue that we can take $C_{a}$ constant without loss of generality. A constant $C_{a}$, however, is incompatible with the fact that $\omega_{a}$ must be covariant with respect to its $\mathrm{SO}(3)_{S^{2}}$ index. In other words, there is no invariant tensor of $\mathrm{SO}(3)_{S^{2}}$ with one index. We conclude $C_{a}=0$. 
Solution for $\sigma_{I J}$. Let us now turn to the determination of $\sigma_{I J}$. We first focus on the components $\sigma_{\hat{I} \hat{J}}$. The equation to solve is

$$
0=d \sigma_{\hat{I} \hat{J}}+\iota_{(\hat{I}} \omega_{\hat{J})}=d \sigma_{\hat{I} \hat{J}}+M_{(I}^{A B} \iota_{J} \bar{\omega}_{A B}^{\mathrm{t}}=d \sigma_{\hat{I} \hat{J}}+M_{(\hat{I}}^{A B} M_{\hat{J})}^{C D} \iota_{C D} \bar{\omega}_{A B}^{\mathrm{t}} .
$$

Making use of (A.22), we see that

$$
\sigma_{\hat{I} \hat{J}}=M_{(\hat{I}}^{A B} M_{\hat{J})}^{C D} \bar{\sigma}_{A B, C D}+u_{\hat{I} \hat{J}},
$$

where $u_{\hat{I} \hat{J}}=u_{\hat{J} \hat{I}}$ are constants.

For $g=0$, we also have to determine $\sigma_{\hat{I} a}$ and $\sigma_{a b}$. The former is determined by the requirement

$$
0=d \sigma_{\hat{I} a}+\frac{1}{2} \iota_{a} \omega_{\hat{I}}+\frac{1}{2} M_{\hat{I}}^{A B} \iota_{A B} \omega_{a} .
$$

Using the formulae for $\omega_{\hat{I}}, \omega_{a}$ given above, as well as the expression of $\stackrel{\circ}{\omega}_{A B}$ and $\stackrel{\circ}{\sigma}_{A B, C D}$ given in (4.5), one verifies the identity

$$
\frac{1}{2} \iota_{a} \omega_{\hat{I}}+\frac{1}{2} M_{\hat{I}}^{A B} \iota_{A B} \omega_{a}=\frac{1}{4} C_{\hat{I}} d z_{a}+d\left[\frac{3 N}{8 \pi^{2}} \cdot \frac{1}{48} z_{a} \epsilon_{A B C D E} M_{\hat{I}}^{A B} q^{C D} y^{E}\right] .
$$

It follows that we can write

$$
\sigma_{\hat{I} a}=-\frac{3 N}{8 \pi^{2}} \cdot \frac{1}{48} z_{a} \epsilon_{A B C D E} M_{\hat{I}}^{A B} q^{C D} y^{E}-\frac{1}{4} C_{\hat{I}} z_{a}+u_{\hat{I} a}
$$

where $u_{\hat{I} a}$ is an arbitrary constant. Once again, however, we must conclude $u_{\hat{I} a}=0$, because there is no $\mathrm{SO}(3)_{S^{2}}$ invariant object with one index $a$. Our final task is the determination of $\sigma_{a b}$ in

$$
0=d \sigma_{a b}+\iota_{(a} \omega_{b)}
$$

We have the identity

$$
\iota_{(a} \omega_{b)}=-d\left[\frac{1}{4} z_{a} z_{b} q^{A B} q^{C D} \bar{\sigma}_{A B, C D}\right]
$$

which implies

$$
\sigma_{a b}=\frac{1}{4} z_{a} z_{b} q^{A B} q^{C D} \bar{\sigma}_{A B, C D}+u_{a b}
$$

for some constant $u_{a b}$. This time there is a natural candidate for a constant $u_{a b}$ compatible with $\mathrm{SO}(3)_{S^{2}}$ symmetry,

$$
u_{a b}=u \delta_{a b}
$$

Summary. The solution for all components of $\omega_{I}, \sigma_{I J}$ is summarized as follows,

$$
\begin{aligned}
\omega_{\hat{I}} & =M_{\hat{I}}^{A B} \bar{\omega}_{A B}^{\mathrm{t}}+2 V_{\Sigma} q^{A B} M_{\hat{I}}^{C D} \bar{\sigma}_{A B, C D}+C_{\hat{I}} V_{\Sigma}, \\
\omega_{a} & =-z_{a}\left[V_{\Sigma} q^{A B} q^{C D} \bar{\sigma}_{A B, C D}+\frac{1}{2} q^{A B} \bar{\omega}_{A B}^{\mathrm{t}}\right], \\
\sigma_{\hat{I} \hat{J}} & =M_{(\hat{I}}^{A B} M_{\hat{J})}^{C D} \bar{\sigma}_{A B, C D}+u_{\hat{I} \hat{J}} \\
\sigma_{\hat{I} a} & =-\frac{1}{2} z_{a} M_{\hat{I}}^{A B} q^{C D} \bar{\sigma}_{A B, C D}-\frac{1}{4} C_{\hat{I}} z_{a}, \\
\sigma_{a b} & =\frac{1}{4} z_{a} z_{b} q^{A B} q^{C D} \bar{\sigma}_{A B, C D}+u \delta_{a b} .
\end{aligned}
$$


If we plug the above relations into (E.8), after some manipulations we recover the expression (5.10) for $E_{4}$ given in the main text. The constants $C_{1}, C_{2}$ in (5.10) are identified with the components of $C_{\hat{I}}$. All terms with $u_{\hat{I} \hat{J}}, u$ are absorbed into $\gamma_{4}$ in (5.10).

\section{F Details on the GMSW setup}

\section{F.1 Brief review of the solutions}

In this appendix we review a class of M-theory solutions with $4 \mathrm{~d} \mathcal{N}=1$ superconformal symmetry, first described in GMSW [20]. The 11d metric reads

$$
\begin{aligned}
d s_{11}^{2} & =L^{2} e^{2 \lambda}\left[d s^{2}\left(A d S_{5}\right)+d s^{2}\left(M_{6}\right)\right], \\
d s^{2}\left(M_{6}\right) & =e^{-6 \lambda}\left[F_{1} d s^{2}\left(C_{1}\right)+F_{2} d s^{2}\left(C_{2}\right)\right]+\frac{e^{-6 \lambda}}{\cos ^{2} \zeta} d y^{2}+\frac{\cos ^{2} \zeta}{9} D \psi^{2}, \\
d D \psi & =-\chi_{1} V_{C_{1}}-\chi_{2} V_{C_{2}} .
\end{aligned}
$$

The constant $L$ is the overall length scale of the solution. The metric on $A d S_{5}$ is normalized in such a way that the Ricci scalar is $R_{\mathrm{AdS}_{5}}=-20$. The spaces $C_{1}, C_{2}$ are two Riemann surfaces, of arbitrary genus. If $C_{i}, i=1,2$, is not a torus, the metric $d s^{2}\left(C_{i}\right)$ is normalized so that the Ricci scalar is $R_{C_{i}}=2 k_{i}$, with $k_{i}= \pm 1$. The symbol $\chi_{i}$ denotes the Euler characteristic of $C_{i}$, while $V_{C_{i}}$ is proportional to the volume form on $C_{i}$. If $C_{i}$ is not a torus, $V_{C_{i}}$ is normalized according to $\int_{C_{i}} V_{C_{i}}=2 \pi$, with no sum over $i$. Notice that, compared to [20], we have reversed the sign of $\psi$.

The quantities $\lambda, F_{i}$ depend on $y$ only and are given by

$$
e^{6 \lambda}=\frac{2\left(a_{1}-k_{1} y^{2}\right)\left(a_{2}-k_{2} y^{2}\right)}{a_{2} k_{1}+a_{1} k_{2}+2 y k_{1} k_{2}\left(y-3 \gamma_{0}\right)}, \quad F_{i}=\frac{1}{3}\left(a_{i}-k_{i} y^{2}\right),
$$

where $a_{i}, \gamma_{0}$ are constants. The quantity $\zeta \in[0, \pi / 2]$ is determined by

$$
e^{3 \lambda} \sin \zeta=2 y
$$

The $G_{4}$ flux takes the form

$$
G_{4}=L^{3}\left[\left(d \gamma_{1} V_{C_{1}}+d \gamma_{2} V_{C_{2}}\right) D \psi-\left(\chi_{1} \gamma_{2}+\chi_{2} \gamma_{1}+\chi_{1} \chi_{2} \gamma_{0}\right) V_{C_{1}} V_{C_{2}}\right],
$$

with the functions $\gamma_{i}$ given as

$$
\begin{aligned}
\gamma_{1} & =\frac{2 a_{2} k_{1} k_{2} y-6 a_{2} k_{1} k_{2} \gamma_{0}+a_{1} y+k_{1} y^{3}}{9 k_{1} k_{2}\left(a_{2}-k_{2} y^{2}\right)} \chi_{1}, \\
\gamma_{2} & =\frac{2 a_{1} k_{1} k_{2} y-6 a_{1} k_{1} k_{2} \gamma_{0}+a_{2} y+k_{2} y^{3}}{9 k_{1} k_{2}\left(a_{1}-k_{1} y^{2}\right)} \chi_{2} .
\end{aligned}
$$

We are adopting conventions in which the quantization of $G_{4}$ flux reads

$$
\int_{\mathcal{C}_{4}} \frac{G_{4}}{\left(2 \pi \ell_{p}\right)^{3}} \in \mathbb{Z},
$$

where $\mathcal{C}_{4}$ is a 4 -cycle and $\ell_{p}$ is the $11 \mathrm{~d}$ Planck length. 
Let us stress that, in this work, we only consider GMSW solutions of the form (F.1), (F.4) in which none of the Riemann surfaces $C_{i}$ is a torus, so that $k_{i} \neq 0$. According to the analysis of [20], in order to a have a regular solution at least one of the two Riemann surfaces must be a sphere. We associate the label 1 to this sphere, while the label 2 is reserved to a Riemann surface of genus $g$, with $g=0$ or $g \geq 2$,

$$
C_{1}=S^{2}, \quad C_{2}=\Sigma_{g} .
$$

We should emphasize that the 4 -form $V_{4}$ in (5.31) is understood to have integral fluxes along 4-cycles. It follows from (F.6) that the relation between $V_{4}$ and $G_{4}$ is

$$
V_{4}=\frac{G_{4}}{\left(2 \pi \ell_{p}\right)^{3}} .
$$

In the main text, we parametrized $V_{4}$ by writing

$$
V_{4}=\left[d \gamma_{\Sigma} \frac{V_{\Sigma}}{2 \pi}+d \gamma_{S^{2}} \frac{V_{S^{2}}}{2 \pi}\right] \frac{D \psi}{2 \pi}-\left[2 \gamma_{\Sigma}+\chi \gamma_{S^{2}}\right] \frac{V_{\Sigma}}{2 \pi} \frac{V_{S^{2}}}{2 \pi} .
$$

Comparison with (F.4) gives the identifications

$$
\gamma_{S^{2}}=\frac{L^{3}}{2 \pi \ell_{p}^{3}}\left(\gamma_{1}+s_{1}\right), \quad \gamma_{\Sigma}=\frac{L^{3}}{2 \pi \ell_{p}^{3}}\left(\gamma_{2}+s_{2}\right), \quad 2 s_{2}+\chi s_{1}=2 \chi \gamma_{0},
$$

where the constants $s_{1,2}$ can be chosen at will. In the text, this ambiguity in the precise definition of $\gamma_{\Sigma}, \gamma_{S^{2}}$ is resolved upon construction of $E_{4}$, when the condition (3.28) is enforced.

The holographic central charge for these solutions was analyzed in [26], where the explicit value of $c$ is derived in the case $\gamma_{0}=0, \Sigma_{g}=S^{2}$. In this situation, one verifies that $N^{\mathrm{N}}=-N^{\mathrm{S}}$, or equivalently $M=0$, see (5.34), (5.37). In the notation of [26] the central charge reads

$$
c=\frac{3^{3 / 2}}{2^{6}} \frac{9(z+1)^{3}-\left(3 z^{2}+4 z+3\right) \sqrt{X}}{z^{3 / 2}} p^{3 / 2} q^{3 / 2} N^{3},
$$

where the parameters $p, q, N$ are related to our quantities $N_{S^{2}}, N_{\Sigma}$ by $N_{S^{2}}=N p$, $N_{\Sigma}=N q$. The objects $X, z$ are defined as

$$
X=9 z^{2}+30 z+9, \quad z=\frac{2 q^{2}-p q+2 p^{2}-2(p-q) \sqrt{p^{2}+p q+q^{2}}}{3 p q} .
$$

In order to compare (F.11) to the central charge (5.60) inferred from the inflow anomaly polynomial, we need the following identity, valid for positive numbers $p, q$,

$$
\left(2 p^{2}-p q+2 q^{2}-2(p-q) \sqrt{p^{2}+p q+q^{2}}\right)^{1 / 2}=q-p+\sqrt{p^{2}+p q+q^{2}} .
$$

With the help of (F.13), the central charge (F.11) can be rewritten as

$$
c=-\frac{9}{16} N^{3}(p+q)\left(2 p^{2}+p q+2 q^{2}\right)+\frac{9}{8} N^{3}\left(p^{2}+p q+q^{2}\right)^{3 / 2},
$$

which indeed matches (5.60) for $\chi=2, M=0$. 


\section{F.2 Details on the construction of $E_{4}$ for GMSW}

Let us first discuss the construction of $V_{4}^{\text {eq }}$. The isometries of $M_{6}$ we gauge are the U(1) symmetry associated to $\psi$ and the $\mathrm{SO}(3)$ symmetry associated to $S^{2}$. The former has background connection $A^{\psi}$, while the latter is associated to $A^{a}$.

The claim that the 4 -form $V_{4}^{\text {eq }}$ is given by

$$
\begin{aligned}
V_{4}^{\mathrm{eq}} & =d\left[\left(\gamma_{\Sigma} \frac{V_{\Sigma}}{2 \pi}+\gamma_{S^{2}} e_{2}^{S^{2}}\right) \frac{(D \psi)^{\mathrm{g}}}{2 \pi}\right] \\
& =\left(d \gamma_{\Sigma} \frac{V_{\Sigma}}{2 \pi}+d \gamma_{S^{2}} e_{2}^{S^{2}}\right) \frac{(D \psi)^{\mathrm{g}}}{2 \pi}+\left(\gamma_{\Sigma} \frac{V_{\Sigma}}{2 \pi}+\gamma_{S^{2}} e_{2}^{S^{2}}\right)\left(-2 e_{2}^{S^{2}}-\chi \frac{V_{\Sigma}}{2 \pi}+2 \frac{F^{\psi}}{2 \pi}\right) .
\end{aligned}
$$

We have exploited the relation

$$
\frac{d(D \psi)^{\mathrm{g}}}{2 \pi}=-2 e_{2}^{S^{2}}-\chi \frac{V_{\Sigma}}{2 \pi}+2 \frac{F^{\psi}}{2 \pi}
$$

which is derived below. The 2 -form $e_{2}^{S^{2}}$ is the global angular form for $\mathrm{SO}(3)$, or equivalently the closed and gauge-invariant completion of $V_{S^{2}} /(2 \pi)$. More explicitly,

$$
e_{2}^{S^{2}}=\frac{1}{8 \pi}\left(\epsilon_{a b c} D z^{a} D z^{b} z^{c}-2 F_{a} z^{a}\right)=\frac{V_{S^{2}}}{2 \pi}-\frac{1}{2} \frac{d\left(z^{a} A_{a}\right)}{2 \pi}, \quad D z^{a}=d z^{a}+\epsilon^{a b c} A_{b} z_{c} .
$$

A useful identity regarding $e_{2}^{S^{2}}$ is the Bott-Cattaneo formula [46],

$$
\int_{S^{2}}\left(e_{2}^{S^{2}}\right)^{2 s+2}=0, \quad \int_{S^{2}}\left(e_{2}^{S^{2}}\right)^{2 s+1}=2^{-2 s}\left[p_{1}(\mathrm{SO}(3))\right]^{s}, \quad s=0,1,2, \ldots
$$

The object $V_{4}^{\text {eq }}$ is manifestly closed and gauge-invariant, and reduces to $V_{4}$ if $F^{\psi}$ and $F^{a}$ are turned off. Moreover, $V_{4}^{\text {eq }}$ is globally well-defined. Indeed, $S^{2}$ does not shrink anywhere on the $y$ interval, and all terms with $(D \psi)^{\mathrm{g}}$ are accompanied by a factor $d y$, so that there is no singularity at the endpoints of the $y$ interval, where $S_{\psi}^{1}$ shrinks.

Before analyzing $V_{4}^{\text {eq }}$ further, let us derive the identity (F.16). If all external connections are turned off,

$$
\frac{D \psi}{2 \pi}=\frac{d \psi}{2 \pi}-2 \frac{A_{S^{2}}}{2 \pi}-\chi \frac{A_{\Sigma}}{2 \pi}, \quad d A_{S^{2}}=V_{S^{2}}, \quad d A_{\Sigma}=V_{\Sigma} .
$$

The 1-forms $A_{S^{2}}, A_{\Sigma}$ are antiderivatives of the volume forms on $S^{2}, \Sigma$, and are only locally defined. The gauging of the 1 -form $d \psi$ is given by

$$
(d \psi)^{\mathrm{g}}=d \psi+2 A^{\psi}+A^{a}\left[z_{a}+2 \epsilon_{a b c} z^{b} \nabla^{\mu} z^{c}\left(A_{S^{2}}\right)_{\mu}\right] .
$$

The index $\mu$ is a curved $2 \mathrm{~d}$ index on $S^{2}$ associated to local coordinates $\zeta^{\mu}$, so that, for example, $d z^{a}=\partial_{\mu} z^{a} d \zeta^{\mu}, g_{\mu \nu}^{S^{2}}=\partial_{\mu} z^{a} \partial_{\nu} z_{a}$. The symbol $\nabla$ is the Levi-Civita connection on $S^{2}$. Notice the appearance in $(d \psi)^{\mathrm{g}}$ of terms proportional to $A^{a}$. They are a consequence of the second term in the Killing 1-form $k_{a}$ in (5.30).

In order to compute $(D \psi)^{\mathrm{g}}$, we also need

$$
\left(A_{S^{2}}\right)^{\mathrm{g}}=\left(A_{S^{2}}\right)_{\mu}\left(d \zeta^{\mu}\right)^{\mathrm{g}}=\left(A_{S^{2}}\right)_{\mu}\left[d \zeta^{\mu}+\epsilon_{a b c} z^{b} \nabla^{\mu} z^{c} A^{a}\right] .
$$


We are now in a position to write

$$
\frac{(D \psi)^{\mathrm{g}}}{2 \pi}=\frac{(d \psi)^{\mathrm{g}}}{2 \pi}-2 \frac{\left(A_{S^{2}}\right)^{\mathrm{g}}}{2 \pi}-\chi \frac{A_{\Sigma}}{2 \pi}=\frac{d \psi}{2 \pi}+2 \frac{A^{\psi}}{2 \pi}-2\left[\frac{A_{S^{2}}}{2 \pi}-\frac{1}{2} \frac{z^{a} A_{a}}{2 \pi}\right]-\chi \frac{A_{\Sigma}}{2 \pi} .
$$

Notice the cancellation of the terms with $\epsilon_{a b c}$ against (F.20) and (F.21). Making use of (F.17), it is now straightforward to check that (F.22) implies (F.16).

In order to make contact with the language of section 3.1 , we have to expand $V_{4}^{\text {eq }}$ is powers of the external connections,

$$
V_{4}^{\mathrm{eq}}=V_{4}^{\mathrm{g}}+F^{\psi} \omega_{\psi}^{\mathrm{g}}+F^{a} \omega_{a}+\left(F^{\psi}\right)^{2} \sigma_{\psi \psi}+F^{a} F^{b} \sigma_{a b}+2 F^{\psi} F^{a} \sigma_{\psi a}
$$

The $\omega$ and $\sigma$ quantities are extracted from comparison with (F.15). In what follows, we only need the expression of $\omega_{\psi}$ and $\omega_{a}$,

$$
\begin{aligned}
\omega_{\psi} & =\frac{2}{2 \pi}\left(\gamma_{\Sigma} \frac{V_{\Sigma}}{2 \pi}+\gamma_{S^{2}} \frac{V_{S^{2}}}{2 \pi}\right) \\
\omega_{a} & =\frac{z_{a}}{2 \pi}\left[-\frac{1}{2}\left(d \gamma_{S^{2}} \frac{D \psi}{2 \pi}-\left(2 \gamma_{\Sigma}+\chi \gamma_{S^{2}}\right) \frac{V_{\Sigma}}{2 \pi}\right)+2 \gamma_{S^{2}} \frac{V_{S^{2}}}{2 \pi}\right] .
\end{aligned}
$$

We verify $\int_{M_{6}} V_{4} \omega_{a}=0$, while we compute

$$
\int_{M_{6}} V_{4} \omega_{\psi}=\frac{2}{2 \pi}\left[\gamma_{S^{2}} \gamma_{\Sigma}\right]_{\mathrm{S}}^{\mathrm{N}}
$$

This quantity must be set to zero. As a result, we can express the four quantities $\gamma_{S^{2}, \Sigma}^{\mathrm{N}, \mathrm{S}}$ in terms of the three flux quanta $N_{S^{2}}, N_{\Sigma}, M$,

$$
\gamma_{S^{2}}^{\mathrm{N}, \mathrm{S}}=\frac{M N_{S^{2}}}{2 N_{\Sigma}-\chi N_{S^{2}}} \pm \frac{1}{2} N_{S^{2}}, \quad \gamma_{\Sigma}^{\mathrm{N}, \mathrm{S}}=-\frac{M N_{\Sigma}}{2 N_{\Sigma}-\chi N_{S^{2}}} \pm \frac{1}{2} N_{\Sigma}
$$

Let us now discuss the equivariant completion of the harmonic 2 -forms $\omega_{\alpha}$ parametrized in (5.38). It is given as

$$
\begin{aligned}
\omega_{\alpha}^{\mathrm{eq}} & =d\left[H_{\alpha} \frac{(D \psi)^{\mathrm{g}}}{2 \pi}\right]+t_{\alpha S^{2}} e_{2}^{S^{2}}+t_{\alpha \Sigma} \frac{V_{\Sigma}}{2 \pi} \\
& =d H_{\alpha} \frac{(D \psi)^{\mathrm{g}}}{2 \pi}+\left(t_{\alpha S^{2}}-2 H_{\alpha}\right) e_{2}^{S^{2}}+\left(t_{\alpha \Sigma}-\chi H_{\alpha}\right) \frac{V_{\Sigma}}{2 \pi}+2 H_{\alpha} \frac{F^{\psi}}{2 \pi} .
\end{aligned}
$$

This is manifestly closed and gauge-invariant and reduces to (5.38) if all external connections are turned off. Moreover, (5.38) is globally defined, since $(D \psi)^{\mathrm{g}}$ is accompanied by $d y$.

Open Access. This article is distributed under the terms of the Creative Commons Attribution License (CC-BY 4.0), which permits any use, distribution and reproduction in any medium, provided the original author(s) and source are credited. 


\section{References}

[1] E. Witten, Some comments on string dynamics, in Future perspectives in string theory. Proceedings, Conference, Strings '95, Los Angeles, CA, U.S.A., 13-18 March 1995, pg. 501 [hep-th/9507121] [INSPIRE].

[2] A. Strominger, Open p-branes, Phys. Lett. B 383 (1996) 44 [hep-th/9512059] [INSPIRE].

[3] J.D. Blum and K.A. Intriligator, New phases of string theory and $6 D$ RG fixed points via branes at orbifold singularities, Nucl. Phys. B 506 (1997) 199 [hep-th/9705044] [INSPIRE].

[4] D. Gaiotto, $N=2$ dualities, JHEP 08 (2012) 034 [arXiv:0904.2715] [INSPIRE].

[5] D. Gaiotto, G.W. Moore and A. Neitzke, Wall-crossing, Hitchin systems and the WKB approximation, arXiv:0907.3987 [INSPIRE].

[6] K. Maruyoshi, M. Taki, S. Terashima and F. Yagi, New Seiberg dualities from $N=2$ dualities, JHEP 09 (2009) 086 [arXiv:0907.2625] [INSPIRE].

[7] F. Benini, Y. Tachikawa and B. Wecht, Sicilian gauge theories and $N=1$ dualities, JHEP 01 (2010) 088 [arXiv:0909.1327] [INSPIRE].

[8] I. Bah and B. Wecht, New $N=1$ superconformal field theories in four dimensions, JHEP 07 (2013) 107 [arXiv:1111.3402] [INSPIRE].

[9] I. Bah, C. Beem, N. Bobev and B. Wecht, AdS/CFT dual pairs from M5-branes on Riemann surfaces, Phys. Rev. D 85 (2012) 121901 [arXiv:1112.5487] [INSPIRE].

[10] I. Bah, C. Beem, N. Bobev and B. Wecht, Four-dimensional SCFTs from M5-branes, JHEP 06 (2012) 005 [arXiv: 1203.0303] [INSPIRE].

[11] D. Anselmi, D.Z. Freedman, M.T. Grisaru and A.A. Johansen, Nonperturbative formulas for central functions of supersymmetric gauge theories, Nucl. Phys. B 526 (1998) 543 [hep-th/9708042] [INSPIRE].

[12] S.M. Kuzenko and S. Theisen, Correlation functions of conserved currents in $N=2$ superconformal theory, Class. Quant. Grav. 17 (2000) 665 [hep-th/9907107] [INSPIRE].

[13] L. Álvarez-Gaumé and E. Witten, Gravitational anomalies, Nucl. Phys. B 234 (1984) 269 [INSPIRE].

[14] L. Álvarez-Gaumé and P.H. Ginsparg, The structure of gauge and gravitational anomalies, Annals Phys. 161 (1985) 423 [Erratum ibid. 171 (1986) 233] [INSPIRE].

[15] W.A. Bardeen and B. Zumino, Consistent and covariant anomalies in gauge and gravitational theories, Nucl. Phys. B 244 (1984) 421 [INSPIRE].

[16] M.J. Duff, J.T. Liu and R. Minasian, Eleven-dimensional origin of string-string duality: a one loop test, Nucl. Phys. B 452 (1995) 261 [hep-th/9506126] [INSPIRE].

[17] E. Witten, Five-brane effective action in M-theory, J. Geom. Phys. 22 (1997) 103 [hep-th/9610234] [InSPIRE].

[18] D. Freed, J.A. Harvey, R. Minasian and G.W. Moore, Gravitational anomaly cancellation for M-theory five-branes, Adv. Theor. Math. Phys. 2 (1998) 601 [hep-th/9803205] [InSPIRE].

[19] J.A. Harvey, R. Minasian and G.W. Moore, Non-Abelian tensor multiplet anomalies, JHEP 09 (1998) 004 [hep-th/9808060] [INSPIRE].

[20] J.P. Gauntlett, D. Martelli, J. Sparks and D. Waldram, Supersymmetric AdS $S_{5}$ solutions of M-theory, Class. Quant. Grav. 21 (2004) 4335 [hep-th/0402153] [INSPIRE]. 
[21] K.A. Intriligator, Anomaly matching and a Hopf-Wess-Zumino term in $6 d, N=(2,0)$ field theories, Nucl. Phys. B 581 (2000) 257 [hep-th/0001205] [inSPIRE].

[22] P. Yi, Anomaly of (2,0) theories, Phys. Rev. D 64 (2001) 106006 [hep-th/0106165] [INSPIRE].

[23] M. Henningson and K. Skenderis, The holographic Weyl anomaly, JHEP 07 (1998) 023 [hep-th/9806087] [INSPIRE].

[24] K. Ohmori, H. Shimizu, Y. Tachikawa and K. Yonekura, Anomaly polynomial of general $6 \mathrm{~d}$ SCFTs, PTEP 2014 (2014) 103B07 [arXiv: 1408.5572] [INSPIRE].

[25] I. Bah, A. Hanany, K. Maruyoshi, S.S. Razamat, Y. Tachikawa and G. Zafrir, $4 d N=1$ from $6 d N=(1,0)$ on a torus with fluxes, JHEP 06 (2017) 022 [arXiv:1702.04740] [INSPIRE].

[26] J.P. Gauntlett, E. O Colgain and O. Varela, Properties of some conformal field theories with $M$-theory duals, JHEP 02 (2007) 049 [hep-th/0611219] [INSPIRE].

[27] E. Witten, On flux quantization in M-theory and the effective action,

J. Geom. Phys. 22 (1997) 1 [hep-th/9609122] [INSPIRE].

[28] J.P. Gauntlett, O.A.P. Mac Conamhna, T. Mateos and D. Waldram, AdS spacetimes from wrapped M5 branes, JHEP 11 (2006) 053 [hep-th/0605146] [INSPIRE].

[29] S. Benvenuti, L.A. Pando Zayas and Y. Tachikawa, Triangle anomalies from Einstein manifolds, Adv. Theor. Math. Phys. 10 (2006) 395 [hep-th/0601054] [INSPIRE].

[30] I. Brunner and A. Karch, Branes and six-dimensional fixed points, Phys. Lett. B 409 (1997) 109 [hep-th/9705022] [INSPIRE].

[31] J.D. Blum and K.A. Intriligator, Consistency conditions for branes at orbifold singularities, Nucl. Phys. B 506 (1997) 223 [hep-th/9705030] [INSPIRE].

[32] K.A. Intriligator, New string theories in six-dimensions via branes at orbifold singularities, Adv. Theor. Math. Phys. 1 (1998) 271 [hep-th/9708117] [InSPIRE].

[33] I. Brunner and A. Karch, Branes at orbifolds versus Hanany Witten in six-dimensions, JHEP 03 (1998) 003 [hep-th/9712143] [INSPIRE].

[34] A. Hanany and A. Zaffaroni, Branes and six-dimensional supersymmetric theories, Nucl. Phys. B 529 (1998) 180 [hep-th/9712145] [INSPIRE].

[35] I. Bah and E. Nardoni, Structure of anomalies of 4d SCFTs from M5-branes and anomaly inflow, JHEP 03 (2019) 024 [arXiv: 1803.00136] [INSPIRE].

[36] K.A. Intriligator and B. Wecht, The exact superconformal $R$ symmetry maximizes a, Nucl. Phys. B 667 (2003) 183 [hep-th/0304128] [InSPIRE].

[37] P. Agarwal, K. Intriligator and J. Song, Infinitely many $N=1$ dualities from $m+1-m=1$, JHEP 10 (2015) 035 [arXiv:1505.00255] [INSPIRE].

[38] M. Fazzi and S. Giacomelli, $N=1$ superconformal theories with $D_{N}$ blocks, Phys. Rev. D 95 (2017) 085010 [arXiv:1609.08156] [InSPIRE].

[39] E. Nardoni, 4d SCFTs from negative-degree line bundles, JHEP 08 (2018) 199 [arXiv: 1611.01229] [INSPIRE].

[40] I. Bah and F. Bonetti, to appear.

[41] I. Bah, F. Bonetti, R. Minasian and E. Nardoni, Class $S$ anomalies from M-theory inflow, Phys. Rev. D 99 (2019) 086020 [arXiv:1812.04016] [INSPIRE]. 
[42] I. Bah, F. Bonetti, R. Minasian and E. Nardoni, Anomaly inflow for M5-branes on punctured Riemann surfaces, JHEP 06 (2019) 123 [arXiv: 1904.07250] [INSPIRE].

[43] D. Gaiotto, A. Kapustin, N. Seiberg and B. Willett, Generalized global symmetries, JHEP 02 (2015) 172 [arXiv: 1412.5148] [INSPIRE].

[44] C. Córdova, T.T. Dumitrescu and K. Intriligator, Exploring 2-group global symmetries, JHEP 02 (2019) 184 [arXiv:1802.04790] [INSPIRE].

[45] M. Libine, Lecture notes on equivariant cohomology, arXiv:0709.3615.

[46] R. Bott and A.S. Cattaneo, Integral invariants of 3-manifolds, J. Diff. Geom. 48 (1998) 91 [dg-ga/9710001]. 